\author{
UNIVERSIDADE DE SÃO PAULO \\ INSTITUTO DE FÍSICA DE SÃO CARLOS
}

RAMON GABRIEL TEIXEIRA ROSA

Assembly, characterization, and validation of a fluorescence lifetime rigid endoscope for clinical imaging of skin lesions

São Carlos 

Assembly, characterization, and validation of a fluorescence lifetime rigid endoscope for clinical imaging of skin lesions

Thesis presented to the Graduate Program in Physics at the Instituto de Física de São Carlos, Universidade de São Paulo to obtain the degree of Doctor of Science.

Concentration area: Applied Physics.

Advisor: Prof. Dr. Cristina Kurachi.

\section{CORRECTED VERSION}

(Original version available on the Program Unit)

São Carlos 
I AUTHORIZE THE REPRODUCTION AND DISSEMINATION OF TOTAL OR PARTIAL COPIES OF THIS DOCUMENT, BY CONVENCIONAL OR ELECTRONIC MEDIA FOR STUDY OR RESEARCH PURPOSE, SINCE IT IS REFERENCED.

Cataloguing data revised by the Library and Information Service of the IFSC, with information provided by the author

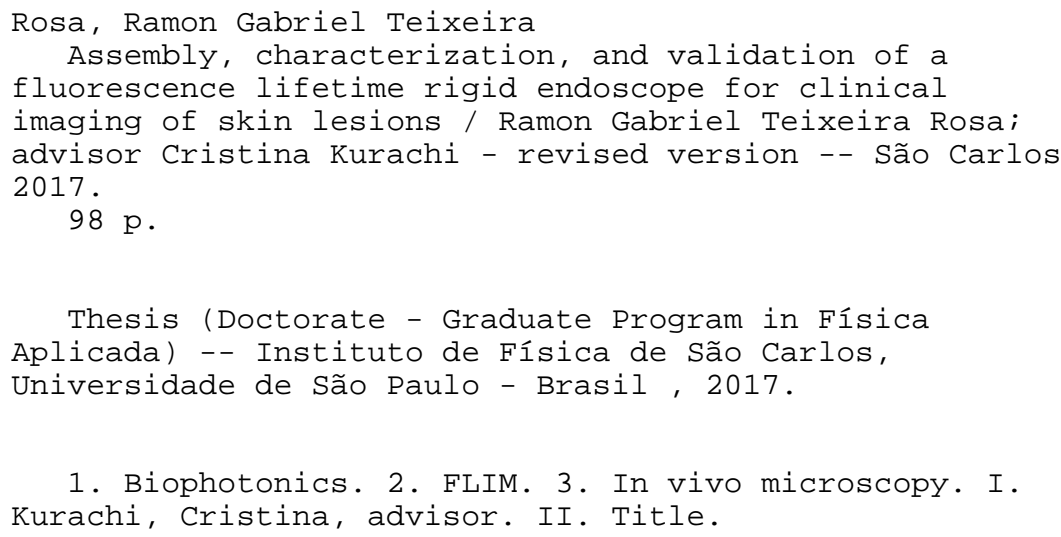




\section{ACKNOWLEDGEMENTS}

I would like to thank my advisor, Prof. Dr. Cristina Kurachi for her guidance, encouragement, and friendship. I thank her for the opportunity to have worked on this project and for everything I could learn from her. I will also be forever thankful for her invaluable support during tough times.

I am also very thankful to Prof. Dr. Javier Jo, who collaborated in this project from its start, for the help, guidance, and friendship. I would not have been able to build the FLIM system without his help. I thank him for receiving me in his lab, for the opportunity to have worked with him and for everything I learned from him.

I am very thankful to Prof. Dr. Vanderlei Bagnato for all the discussions, ideas, support and encouragement.

I am especially grateful to Dr. Sebastião Pratavieira for introducing me to the optical microscopy principles, for all the help during this project, the ideas and discussions, and for the friendship.

I want to thank Dr. Natalia Inada for the help with the Ethics Committe proposal, for the discussions, and for the friendship.

I would like to thank Dr. Lilian Moriyama for the discussions, the help, the friendship, and the support.

My deep gratitude goes to Dr. Ana Gabriela Salvio, for the availability and dedication with the Ethics Committee proposal and the clinical study.

I am very thankful to my good friend Leandro Pingueiro. Without a doubt, the electrical and mechanical components of the FLIM system would not look or work nearly as good if it was not for his help. I am very helpful for the countless hours he spent helping me on this project and for teaching me the basic of machining.

I would like to thank Renan Romano for the help with the FLIM in vivo measurements and for the friendship.

I want to express my gratitude to Cintia Teles, Clara Faria, ilaialI Leite, Layla Pires, and Michelle Requena for the help with the animal model.

I would like to thank the Optics Group secretary staff for all the assistance provided over those years.

I am thankful to all the staff of the Sao Carlos Institute of Physics machine shop and of the Laboratório de Instrumentação Eletrônica (LIEPO), especially João Marcelo for the help.

I would like to thank the librarians of the Sao Carlos Institute of Physics, especially Maria Neusa de Aguiar Azevedo, for the careful review of this thesis. 
My profound gratitude goes to the Laboratory for Optical Diagnosis and Imaging (Texas A\&M University) staff, especially to Dr. Shuna Cheng, for all the help and for teaching me so many things, and to Jorge Palma, for the friendship and hospitality.

I would like to express my gratitude to my father for his love and faith in me. He gifted me a small microscope when I was a kid only to make me more and more curious, and never would I have imagined I was going building a microscope on my own someday.

I would like to thank my mother for the everlasting love and care. I will be forever thankful for all her lessons and for the encouragement. Her memory will be forever in my heart.

Finally, I would like to thank the love of my life, Franciele Renata Henrique, who has been my pillar of strength and my pride and joy. Not only she held my hands during the hardest moments of my life and kept me from drowning, but also provided me some of the best and brightest moments of my life. I want to thank her for the love, care and support during almost one third of my life now. I thank her for keeping me inspired and amazed, and for some of the best and absolutely worst jokes ever told in human history.

I want to thank Capes, CNPq (PVE 401150/2014-3, INCT-INOF), and FAPESP (CePOF) for the financial support. 
"I disagree strongly with whatever work this quote is attached to."

- Randall Munroe. 



\section{ABSTRACT}

ROSA, R. G. T. Assembly, characterization, and validation of a fluorescence lifetime rigid endoscope for clinical imaging of skin lesions. 2017. 98 p. Thesis (Doctor of Science) - Instituto de Física de São Carlos, Universidade de São Paulo, São Carlos, 2017.

Fluorescence based microscopy techniques have been extensively used in biological sciences. The most common approach is the steady-state fluorescence microscopy. Although the said approach is powerful, it often lacks sensitivity to detect several biochemical processes that may indicate relevant conditions of biological tissues. The fluorescence dynamics analysis not only brings intrinsic information about the tissue, but is also less sensitive to the medium scattering and absorption, and sometimes capable of distinguishing between fluorescent structures with indistinguishable spectra. The intrinsic fluorescence lifetime of biological tissues is usually affected by some clinical conditions, especially when those conditions cause or are correlated with metabolic modifications. Time-resolved spectroscopy techniques can be used to detect those modifications and may be used as a tool to improve the detection and diagnosis rate of such conditions. Fluorescence Lifetime Imaging Microscopy (FLIM) combines the temporal resolution and the microscopy concept, so fluorescence lifetime images can be generated. This technique has a great potential for clinical applications since it may be able to detected lesions and delineate its borders. However, FLIM usually demands a more sophisticated instrumentation than most techniques based on the steady-state approach, what creates a difficulty for moving such a system to a clinical setting. We report the assembly, characterization, validation, and clinical application of a multispectral FLIM system featuring a handheld probe composed of a laser scanning rigid endoscope. The assembled system uses a $355 \mathrm{~nm}$ short pulsed laser as excitation and has three spectral channels, targeting the emission of collagen, NADH, and FAD, which are important endogenous fluorophores. The system acquires images of $8.65 \times 8.65 \mathrm{~mm}^{2}$ areas in $\sim 2.4 \mathrm{~s}$. MATLAB codes were written to process the images using a biexponential model and a modified phasor approach. In vivo validation measurements of tumors induced in mice were performed. The system was also validated with in vivo imaging of skin of healthy volunteers. The assembled FLIM system was moved to Hospital Amaral Carvalho, where we performed a pilot clinical study, in which different types of skin lesions were imaged in vivo in a clinical setting. A significant contrast was achieved on seborrheic keratosis, Bowen's disease, and sclerodermiform basal cell carcinoma tumors. These results indicate the potential of this technique for clinical imaging of skin lesions.

Keywords: Biophotonics. FLIM. Microscopy. 



\section{RESUMO}

ROSA, R. G. T. Montagem, caracterização e validação de um endoscópio rígido de tempo de vida de fluorescência para imageamento clínico de lesões de pele.. 2017. 98 p. Tese (Doutorado em Ciências) - Instituto de Física de São Carlos, Universidade de São Paulo, São Carlos, 2017.

Técnicas de microscopia baseadas em fluorescência têm sido extensamente utilizadas em ciências biológicas. A abordagem mais comum se baseia na microscopia de fluorescência de estado estacionário. Apesar de poderosa, essa abordagem frequentemente não apresenta sensibilidade suficiente para detectar diversos processos bioquímicos que podem ser indicadores de relevantes problemas em tecidos biológicos. A análise da dinâmica da fluorescência não apenas trás informações intrínsecas sobre o tecido, mas também é menos sensível a espalhamento e absorção pelo meio, além de ser capaz de distinguir entre estruturas fluorescentes com espectros indistinguíveis em alguns casos. O tempo de vida intrínseco de tecidos biológicos é normalmente afetado por condições clínicas, especialmente quando estas condições causam ou são relacionadas a modificações metabólicas. As técnicas de espectroscopia resolvidas no tempo podem detectar essas modificações e podem ser utilizadas como uma ferramenta para melhorar a detecção e o diagnóstico dessas condições. A Microscopia de Tempo de Vida de Fluorescência (FLIM) combina a resolução temporal ao conceito de microscopia, de forma que imagens de tempos de vida de fluorescência podem ser gerados. Essa técnica tem um grande potencial para aplicações clínicas uma vez que ela pode ser capaz de detectar lesões e delinear suas bordas. No entanto, FLIM requer uma instrumentação muito mais sofisticada do que a maior parte das técnicas baseadas no estado estacionário, o que cria uma dificuldade para que tais sistemas possam ser levados a ambientes clínicos. Nós reportamos a montagem, caracterização, validação e aplicação clínica de um sistema FLIM multiespectral com uma sonda manual composta de um endoscópio rígido de varredura laser. O sistema montado utiliza um laser pulsado de $355 \mathrm{~nm}$ como fonte de excitação e conta com três canais espectrais, visando a emissão do colágeno, do NADH e do FAD, três importantes fluoróforos endógenos. O sistema é capaz de adquirir imagens de áreas de $8,65 \times 8,65 \mathrm{~mm}^{2}$ em $\sim 2,4 \mathrm{~s}$. Códigos em MATLAB foram escritos para processar as imagens usando um modelo biexponencial e uma abordagem modificada dos fasores. Medidas in vivo de tumores induzidos em camundongos foram realizadas para validação do sistema. O sistema também foi validado com a realização de medidas in vivo da pele de voluntários sadios. O sistema montado foi levado ao Hospital Amaral Carvalho, onde realizamos um teste clínico piloto no qual diferentes tipos de lesões de pele foram imageados in vivo em um ambiente clínico. Um contraste significante foi alcançado em tumores de queratose seborreica, doença de Bowen e carcinoma basocelular escleroder- 
miforme. Esses resultados indicam o potencial desta técnica para o imageamento clínico de lesões de pele.

Keywords: Biofotônica. FLIM. Microscopia. 


\section{LIST OF ABREVIATIONS AND ACRONYMS}

\begin{tabular}{|c|c|}
\hline $\mathrm{BCC}$ & Basal Cell Carcinoma \\
\hline $\mathrm{CCD}$ & Carge-Coupled Device \\
\hline CONEP & Conselho Nacional de Ética em Pesquisas \\
\hline DTFT & Discrete Time Fourier Transform \\
\hline EFL & Effective Focal Lenght \\
\hline FAD & Flavin Adenin Dinucleotide \\
\hline FLIM & Fluorescence Lifetime Imaging Microscopy \\
\hline FOV & Field-of-View \\
\hline FPGA & Field-Programmable Gate Array \\
\hline FRET & Förster Resonance Energy Transfer \\
\hline FWHM & Full Width at Half Maximum \\
\hline GOI & Gated Optical Intensifier \\
\hline GRIN & Graded-Index \\
\hline GUI & Graphical User Interface \\
\hline ICCD & Intensified Charge-Coupled Device \\
\hline iDTFT & Inverse Discrete Time Fourier Transform \\
\hline IRF & Instrument Response Function \\
\hline MCP-PMT & Microchannel Plate Photomultiplier Tube \\
\hline NADH & Reduced Nicotinamide Adenine Dinucleotide \\
\hline $\mathrm{OCT}$ & Optical Coherence Tomography \\
\hline PMT & Photomultiplier Tube \\
\hline PSD & Power Spectral Density \\
\hline $\mathrm{SCC}$ & Scamous Cell Carcinoma \\
\hline SNR & Signal-to-Noise Ratio \\
\hline SVM & Support Vector Machine \\
\hline TCSPC & Time-Correlated Single Photon Counting \\
\hline
\end{tabular}





\section{Contents}

1 Introduction $\quad 15$

2 Objectives $\quad 23$

2.1 General objectives . . . . . . . . . . . . . . . . . . 23

2.2 Specific objectives . . . . . . . . . . . . . . . . . . . . 23

3 Time-resolved fluorescence spectroscopy and microscopy 25

3.1 Basic principles of fluorescence . . . . . . . . . . . . . . . . . 25

3.2 Temporal behavior of fluorescence . . . . . . . . . . . . . . 26

3.3 Time-resolved fluorescence spectroscopy . . . . . . . . . . . . . . . . . 29

3.4 Lifetime estimation and fluorescence signal deconvolution . . . . . . . . 33

3.4.1 Fast lifetime estimation from raw signal . . . . . . . . . . . 34

3.4 .2 Deconvolution methods . . . . . . . . . . . . . . 37

3.5 Fluorescence Lifetime Imaging Microscopy . . . . . . . . . . . . . . . . . 40

4 Material and methods $\quad 43$

4.1 FLIM setup . . . . . . . . . . . . . . . . . . . . 43

4.2 Control and acquisition . . . . . . . . . . . . . . . . . . . 48

4.3 Handheld probe design and optimization . . . . . . . . . . . . . . 49

4.4 FLIM data analysis . . . . . . . . . . . . . . . . . . . . . . 52

4.5 On-the-bench setup . . . . . . . . . . . . . . . . 53

4.6 Mobile platform for the FLIM system . . . . . . . . . . . . . . . . . . 54

4.7 In vivo imaging . . . . . . . . . . . . . . . . . . . . . . . . . . 56

5 Results and discussions $\quad 61$

5.1 On-the-bench setup . . . . . . . . . . . . . . . 61

5.1 .1 IRF characterization . . . . . . . . . . . . . . 61

5.1 .2 Imaging . . . . . . . . . . . . . . . . . 62 
5.2 Mobile platform multispectral FLIM system . . . . . . . . . . . . . . 64

5.2.1 Channels spectral efficiency . . . . . . . . . . . . . 64

5.2 .2 IRF characterization . . . . . . . . . . . . . 65

5.2 .3 Measurements at the animal model . . . . . . . . . . . . . 68

5.2 .4 Validation with human skin imaging . . . . . . . . . . 73

5.3 Clinical study . . . . . . . . . . . . . . . . . . 76

$\begin{array}{llr}6 & \text { Conclusions } & 87\end{array}$

$\begin{array}{lr}\text { References } & 89\end{array}$

ANNEX A - Research Ethics Committee approval 95

ANNEX B - Research Ethics Committee approval $\quad 97$ 


\section{Introduction}

Cancer is a major public health problem worldwide, being the second leading cause of death in the world, several cancer types were responsible for 8.8 million registered deaths in 2015 and it is estimated that 1 in 6 deaths occur due to complications associated to it. The number of new cases is expected to rise by approximately $70 \%$ over the next two decades. ${ }^{1}$

One of the key points to address this issue and reduce cancer mortality rate is the early treatment of the disease, as cancer is much more likely to respond effectively to treatment, increasing the chances of survival, reduced morbidity rate, besides reducing the overall treatment cost. The first step in delivering early treatment is to provide early and accurate diagnosis.

The gold standard diagnosis method depends on the type of cancer, but it is usually based on cytology or hystopathology analysis. Some cases, however, can be diagnosed by a simple clinical inspection by a trained professional. All those methods, however, strongly rely on the experience of the medical doctor carrying the clinical examination and biopsy, as well as of the pathologist.

Additionally, cytology and histopathology analysis demand invasive procedures, may be time consuming and often require multiple sample preparation methods, like microslicing, fixing and staining with expensive biomarkers. The sample preparation and diagnostic methods are performed at a laboratory facility, so the clinician does not have the lesion classification at the same day.

The early detection problem, in the case of solid tumors, does not lie only on the fast and accurate diagnostics of the tumor tissue, but also on the localization of the tumor in the first place. Many methods can be used to locate solid tumors, like visual inspection, palpation and x-ray imaging, but those methods often lack the sensitivity to detect the early stage tumors ${ }^{2}$ and contrast to distinguish the tumor from surrounding healthy tissue. ${ }^{3}$

In this sense, main limitations of the present diagnostic methods are: (1) invasive procedure, where there is a need of tissue removal; (2) laboratory processing, requiring designated facility and personnel and not allowing fast result at clinical setting; (3) sample destructive procedure, not allowing tissue monitoring during treatment, multiple 
biopsies from the same lesion, nor screening of large lesions.

Optical techniques, based on light tissue interactions, as absorption, scattering and luminescence, have been presented as auxiliary detection methods addressing these limitations.

During the last decades there has been a noticeable increase on the use of fluorescence based techniques in biological sciences. Their applications range from DNA sequencing and flow cytometry to forensics and medical diagnostics. The use of those techniques is rapidly expanding because they can bring valuable biochemical and structural information of tissues. They are also usually nondestructive and suitable for in vivo applications. ${ }^{4}$

Some biological molecules have intrinsic fluorescent properties. This intrinsic fluorescence, called autofluorescence or native fluorescence, has been studied since the 16th century. ${ }^{5}$ Since the invention of the fluorescence microscope in 1908 by Köhler and Seidentopf, ${ }^{6}$ cells and tissues started to be studied through related techniques. Those studies presented a significant breakthrough in the comprehension of molecular interactions and signaling processes in cells and tissues. Fluorescence based techniques started to be used to infer biochemical, functional and structural properties of fluorescent biomolecules without the need of administration of a contrast agent. Since those properties can be modified by pathological changes and therapeutic interventions, ${ }^{7}$ those techniques began to be applied in diagnostics and were shown to improve detection rate. ${ }^{2}$

Biochemical changes induced by pathology progression precede the morphological alterations, so a diagnostic technique that may monitor the biochemical tissue status in a noninvasive way is highly attractive, especially when considering early detection of cancer lesions. Fluorescence based techniques have shown great potential for this application. $^{7}$

While standard steady-state fluorescence spectroscopy can be used to access meaningful information about different types of samples, it does not provide essential information that is related to the fluorescence temporal dynamics. Through time-resolved fluorescence spectroscopy it is possible to investigate those properties and obtain contrast for imaging techniques such as in Fluorescence Lifetime Imaging Microscopy (FLIM).

Fluorescence lifetime is a property of the fluorophore in a given medium. It is an intensive property which was shown not to be affected in many cases by the measurement method or initial perturbation conditions like excitation duration, ${ }^{8}$ by photobleaching, ${ }^{9}$ by the presence of absorbers in the sample or by fluorescence signal scattering. ${ }^{7}$ As many endogenous fluorophores in biological tissues have overlapping fluorescence spectra, ${ }^{2,10,11}$ (Figure 1.1) the fluorescence lifetime can help on providing additional discrimination 
parameters.

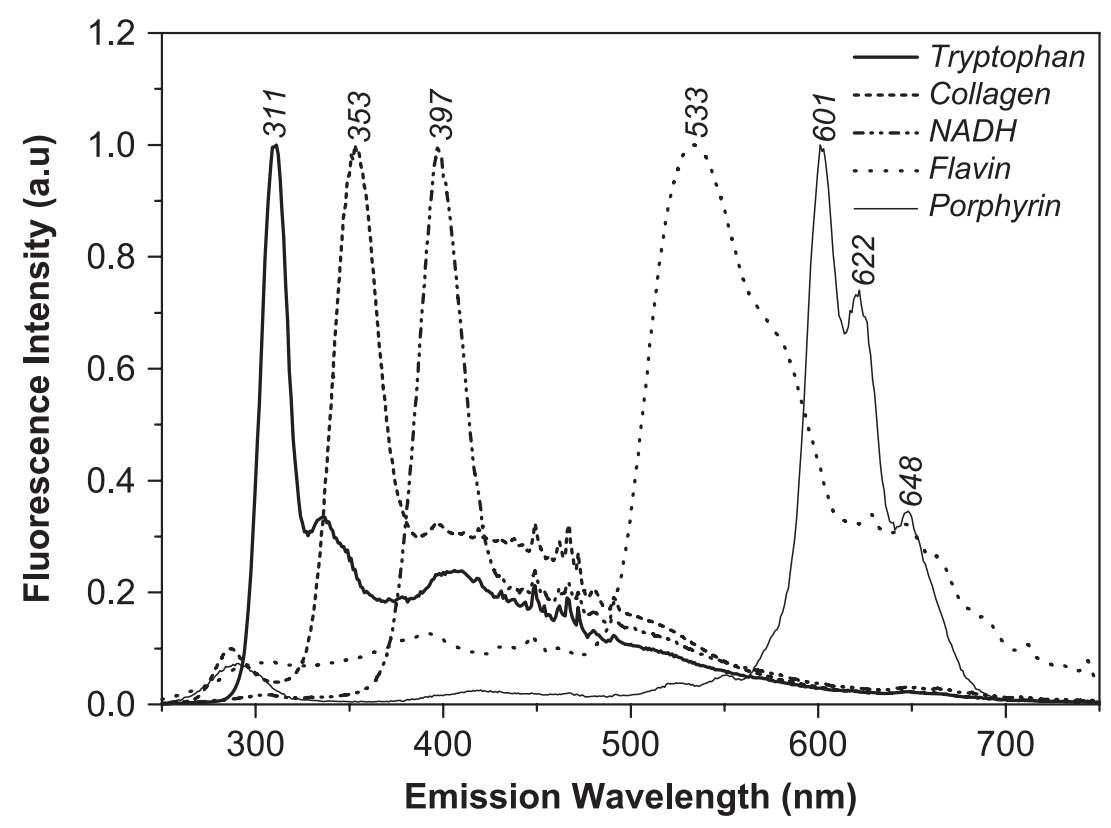

Figure 1.1 - Stokes shift spectra of some important endogenous biological fluorophores. Source: EBENEZAR ${ }^{12}$

The fluorescence lifetime also depends on the properties of the molecular environment in which the fluorophore is present, like $\mathrm{pH}$, partial pressure of oxygen, ion concentration, and others. On the other hand, fluorescence intensity depends primarily on fluorophore concentration and quantum yield, but can also be affected by the medium absorption and scattering, so fluorescence lifetime imaging has the advantage of allowing the construction of biological microenvironment maps for quantitative measurements of properties that may be independent of fluorophore concentration. Special consideration must be performed when interrogation tissue samples and not monolayered in vitro cells.

In living tissues, there are sets of fluorophores contributing to the signal that are usually not found on in vitro cells samples, like collagen and keratin. There are also increased absorption by molecules that may be present (e.g. melanin) and scattering on the interface between tissue layers and due to tissue heterogeneity. All those effects strongly influence the intensity and the spectrum of the measured fluorescence signal. Additionally, there may be multiple types of cells with different optical properties contributing to the fluorescence signal. For this reason, the fluorescence based analysis of living tissues is often based on quantitative statistical classification.

Due to its remarkable capability of accessing intrinsic information of samples, FLIM has been extensively used in biological sciences. Through time-resolved fluorescence spectroscopy imaging techniques, it is possible to obtain more parameters to produce contrast. ${ }^{7}$ As other fluorescence based techniques, FLIM is noninvasive and the technique is suitable for in vivo applications per se. However, FLIM measurements require a more complex instrumentation when compared to the ones based on steady-state fluorescence 
data, creating intrinsic difficulties for its application in in vivo imaging, specially in clinical environments. An effort is being invested in the development of FLIM systems capable of performing in vivo analysis.

Fruhwirth et al. ${ }^{13}$ (2010) developed a fluorescence lifetime endoscopy system using the Time Correlated Single Photon Counting (TCSPC) technique. An endoscope based on a coherent optical fiber bundle was used. The collection of a single spectral channel $(510 \pm 10 \mathrm{~nm})$ was performed using an excitation at $473 \mathrm{~nm}$. The imaging was performed using an afocal laser scanning system and the collection was done in a non-descanned geometry by a Photomultiplier Tube (PMT). This system presented a lateral resolution of about $5 \mu \mathrm{m}$, allowing single cells visualization, and a field-of-view (FOV) of $600 \mu \mathrm{m}$. It was successfully employed on the imaging of in vitro live cells, where Förster Resonance Energy Transfer (FRET) was observed. The authors concluded that those results demonstrate a great potential for this technique to be employed on in vivo clinical applications.

Kennedy et al. ${ }^{14,15}(2010,2011)$ also developed a confocal FLIM endoscope based on an available commercial endomicroscope. This system uses a coherent optical fiber bundle endoscope, a laser scanning system and TCSPC acquisition modules. A frequencydoubled Ti:Sapphire laser, with center wavelength of $488 \mathrm{~nm}$ was used as excitation source. The collection of a single spectral channel, where the emission wavelengths were filtered, was performed by a PMT on a descanned confocal geometry. This system presented a lateral resolution of $1.4 \mu \mathrm{m}$ and a FOV of $240 \mu \mathrm{m}$. In vitro labelled cells, stained pollen grains, and unstained ex vivo tissue were successfully imaged with this system, and FRET could be observed.

A wide-field FLIM flexible coherent optical fiber bundle endoscope based on gated optical image intensifier was developed by Munro et al. $(2005)^{16}$ In this system, the whole field-of-view is excited by a short laser pulse and a Gated Optical Intensifier (GOI) coupled to a Charge-Coupled Device (CCD) is used to record the fluorescence intensity with spatial resolution at a specified time after the excitation pulse. By varying the delay between pulse and fluorescence intensity recording, it is possible to reconstruct fluorescence decay curves for all pixels. The excitation source for this system is a $355 \mathrm{~nm}$ laser and the collection was done by a single spectral channel filtered by a long pass filter with edge at $375 \mathrm{~nm}$. This system was successfully employed on the imaging of unstained ex vivo tissue samples.

Requejo-Isidro et $a l .{ }^{17}$ (2004) developed a wide-field rigid endoscope FLIM system based on a commercial arthroscope. This system also uses a GOI coupled to a CCD to retrieve temporal information from the sample and, a $355 \mathrm{~nm}$ pulsed laser excitation and collection by a single spectral channel filtered by a $435 \mathrm{~nm}$ long pass filter. This system presented a lateral resolution of approximately $50 \mu \mathrm{m}$ and FOV of $32 \times 22 \mathrm{~mm}^{2}$ 
at a working distance of $4.5 \mathrm{~cm}$. The authors demonstrated this system is well suited for imaging of ex vivo bisected lamb kidney samples.

Elson et al. ${ }^{18}$ (2007) reported the development of a side-viewing optical fiber-based flexible FLIM endoscope for intravascular applications. The endoscope is composed of a coherent optical fiber bundle terminated with a Graded-Index (GRIN) lens cemented to a 90 degree prism to allow side viewing. A $337 \mathrm{~nm}$ Nitrogen laser was used as excitation source. The excitation was coupled to a separate optical fiber whose distal end was positioned to overlap the illumination with the field-of-view of the collection endoscope. The collected signal was filtered by one of three available band pass filters $(390 \pm 35 \mathrm{~nm}$, $450 \pm 32 \mathrm{~nm}$, and $550 \pm 50 \mathrm{~nm}$ ). The filters were placed on a filter wheel at the distal end of the fiber bundle so the spectral band to be collected could be easily chosen. The image recording was performed by a GOI coupled to a CCD. This system presented a FOV of $4 \mathrm{~mm}$ and spatial resolution of approximately $50 \mu \mathrm{m}$. The system was successfully used to image freshly excised, unstained rat aortic and pig coronary, and aortic arteries, being able to distinguish between elastin-rich and collagen-rich region of the tissue.

Mizeret et al. ${ }^{3}$ (1999) reported the development of a fluorescence lifetime imaging rigid endoscope using the frequency-domain approach. A tunable Argon ion laser was used as excitation source at two wavelengths: $488 \mathrm{~nm}$ and $514 \mathrm{~nm}$. A spectral channel (515 $\mathrm{nm}$ long pass for $488 \mathrm{~nm}$ excitation, and $550 \mathrm{~nm}$ long pass for $514 \mathrm{~nm}$ excitation) was acquired by a CCD after amplification by an image intensifier tube. The lateral resolution of this system was described in terms of its modulation transfer function, which has a spatial frequency bandwidth (at 20\%) of $1.22 \mathrm{~mm}^{-} 1$. From the parameters presented in this paper, we estimate that the FOV of this endoscope is around $20 \mathrm{~mm}$. A pilot test was performed to demonstrate the suitability of this system for in vivo imaging of human healthy bronchial mucosa.

Cheng et al. ${ }^{19}$ (2013) reported the development of a multispectral time-domain FLIM endoscopy system suitable for in vivo imaging. The endoscope is composed of a coherent optical fiber bundle terminated with a GRIN lens. A $355 \mathrm{~nm}$ pulsed laser is used as excitation. The collected signal is split in three spectral channels by a combination of dichroic beam splitters and filters. The image of the fiber bundle related to each spectral channel is projected on a different region of an Intensified CCD (ICCD) so that they are recorded simultaneously, which is very important for in vivo applications to avoid motion artifacts. The three spectral channels were chosen to be $390 \pm 20 \mathrm{~nm}, 450 \pm 20 \mathrm{~nm}$, and $560 \pm 20 \mathrm{~nm}$ based on the emission peak of three important biological molecules, which are the collagen, the reduced nicotinamide adenine dinucleotide (NADH) and the flavin adenin dinucleotide (FAD). The system was successfully tested with in vivo imaging of a lesion from a hamster cheek pouch that was previously diagnosed as dysplasia. The results presented in this paper show that this technique is capable of distinguishing the 
malignant lesion region from the healthy tissue surroundings.

Shrestha et $a .^{20}$ (2010) reported the development of multispectral table top FLIM systems for in vivo applications. This system uses a $355 \mathrm{~nm}$ laser as excitation source and a single lens laser scanning system. The collected signal is split in three spectral channels $(390 \pm 20 \mathrm{~nm}, 450 \pm 20 \mathrm{~nm}$, and $550 \pm 20 \mathrm{~nm}$, also aiming the collagen, NADH and FAD peak emissions). The signal from each spectral channel is coupled into fibers with different lengths so different delay is included on it. This creates a temporal displacement between each spectral channel so they can be sequentially recorded by the same fast Microchannel Plate Photomultiplier Tube (MCP-PMT). This allowed fast multispectral FLIM imaging, having a FOV of up to $4 \mathrm{~mm}$ and lateral resolution of around $100 \mu \mathrm{m}$. This system was also validated with in vivo imaging of malignant lesions of hamster cheek pouch, where the dysplastic regions could be clearly distinguished from healthy areas. A similar system ${ }^{21}$ was also used for the autofluorescence based biochemical characterization of ex vivo atherosclerotic plaques, where collagen-rich and lipid-rich areas were successfully indicated. Another similar assembly ${ }^{22}$ featuring a helical scanning side-viewing endoscope was developed to intravascular imaging of atherosclerotic plaques. This system was validated with measurements on pig aorta phantom.

Cheng et al. ${ }^{23,24}$ (2014) presented the development of a multispectral FLIM system featuring a rigid handheld endoscope for in vivo imaging. The system uses a frequencytripled Nd:YAG laser $(355 \mathrm{~nm})$ as excitation source. The excitation is guided to a handheld probe that performs the laser scanning, collects the fluorescence signal, and couples it in another optical fiber. The fluorescence signal is split in three spectral channels by a set of dichroic mirros and filters and coupled in three fibers with different lengths acting as optical delay lines. The fluorescence signal is measured by a fast MCP-PMT and read by a digitizer. The selected bands for the spectral channels are: $390 \pm 20 \mathrm{~nm}, 452 \pm 23 \mathrm{~nm}$, and long pass at $550 \mathrm{~nm}$. The three spectral channels were also chosen having in mind the peak emission of collagen, NADH, and FAD. Some different configurations of the probe were tested, which presented lateral resolution ranging from $35 \mu \mathrm{m}$ to $140 \mu \mathrm{m}$, and FOV ranging from $6.5 \mathrm{~mm}$ to $13 \mathrm{~mm}$. This system was applied on the imaging of in vivo hamster cheek pouch, ex vivo human oral biopsy, and in vivo ventral region of human tongue. This paper presented the first demonstration of a time-domain multispectral FLIM endoscope that requires only one excitation pulse for the acquisition of each pixel and its viability to in vivo imaging. This greatly increases the acquisition speed, reducing motion artifacts and reducing the overall exposure of the sample to ultraviolet light. More recently, Cheng et al. ${ }^{25}$ (2016) reported the application of this system in the clinical evaluation of oral epithelial cancer and pre-cancer lesions. The lesions were imaged using the presented system before the biopsy was collected by the physician. A Support Vector Machine (SVM) classifier was trained and applied to each pixel of the image to generate classification 
maps distinguishing benign from malignant lesions. The results show a high correlation between the FLIM/SVM classification and the histopathology analysis, what indicates this technique has a great potential for the early detection of some malignant lesions.

Other imaging techniques were also combined with FLIM to improve the potential for the analysis of tissues. Park et al. ${ }^{26}$ (2010) and Jo et al. ${ }^{27}$ (2010) developed FLIM microscopes combined to Optical Coherence Tomography (OCT) systems to allow simultaneous biochemical and morphological characterization of tissues. Another system to allow simultaneous biochemical and morphological characterization was developed by Jabbour et $a l .{ }^{28}$ (2013) It combined combined a Reflectance Confocal Microscope with a FLIM microscope. Those systems have been successfully used to study distinct oral mucosa conditions.

De Beule et al. ${ }^{29}$ (2007) reported the development of a hyperspectral fluorescence lifetime probe for skin cancer diagnosis. This system uses two fast pulsed lasers (355 $\mathrm{nm}$ and $440 \mathrm{~nm}$ ) as excitation and has hyperspectral module composed of a spectrograph and a 16 channels-PMT. An optical fiber based probe to provide both illumination and fluorescence collection. Fresh ex vivo human skin lesions were measured by the developed system. The results indicate the potential of this technique on the discrimination of Basal Cell Carcinoma (BCC) skin cancer from healthy tissues.

Galletly et $a l .{ }^{30}$ (2008) report the analysis of ex vivo human skin cancer with fluorescence lifetime imaging using $355 \mathrm{~nm}$ excitation. The results shown that this technique can help on the detection and delineation of the borders of human BCC and indicate the potential future role of this technique.

Nogueira et al. ${ }^{31,32}(2015,2017)$ developed a portable fluorescence lifetime spectroscopy system for in vivo interrogation of skin cancer lesions. The system uses also uses two excitation sources at $378 \mathrm{~nm}$ and $445 \mathrm{~nm}$, and collects multiple spectral channels using a set of exchangeable filters. This system has a probe composed of a bundle of excitation and collection optical fibers that allows in situ interrogation of the skin lesions. The results indicate that this technique can be used to differentiate lesions with clinically similar lesions features. However, this system does not allow imaging, what creates a difficulty in terms of lesion borders delineation.

Based on the potential diagnostic resolution of FLIM to discriminate cancer lesions and on the lack of available clinical systems, our main objective in this study was to assemble, characterize and validate a FLIM system to finally apply for in vivo imaging.

In this thesis, we present the assembly, characterization and validation and in vivo application of a fast multispectral FLIM endoscope designed for use in clinical setting. 
The system was built on a mobile cart which could be taken to both an animal experimentation laboratory and a clinical center of Hospital Amaral Carvalho (Jahu, SP, Brazil), a hospital focused on the diagnosis and treatment of cancer, where measurements could be carried out to verify the potential of this technique for the detection of different types of skin lesions and conditions. The FLIM system features a handheld probe that can be used to image hard-to-reach regions and was originally designed for the screening of cervix cancer, but is being initially tested on the screening of skin lesions.

In the following chapters, we will discuss the basic theory behind fluorescence, fluorescence lifetimes, time-resolved fluorescence spectroscopy data analysis, and the main concepts regarding fluorescence lifetime imaging. We will also present the setup of the FLIM system we built, discussing control and acquisition details, the steps for designing and optimizing the handheld probe, the validation measurements of the system, and the results of the in vivo clinical study. Finally, we will present the conclusions of our work. 


\section{Objectives}

Our general and specific objectives are described below.

\section{$2.1 \quad$ General objectives}

Our general objective is to build a FLIM system suitable for clinical applications and perform a clinical study evaluating its performance on the imaging and detection of skin lesions.

\section{$2.2 \quad$ Specific objectives}

Our specific objectives are:

- assembly, characterization, and optimization of a simplified bench-top FLIM system;

- development of control and acquisition interface;

- assembly, characterization, and optimization of the multispectral module;

- design, simulation, optimization, assembly, and characterization of a handheld probe;

- design and assembly of a mobile platform to allow the transportation of the FLIM system;

- characterization of the mobile FLIM system;

- validation of the FLIM system with animal models;

- clinical study: in vivo imaging of skin lesions. 


\section{Time-resolved fluorescence spectroscopy and microscopy}

\subsection{Basic principles of fluorescence}

The fluorescence phenomenon results from the process that some substances undergo after being excited by light. After absorbing a photon with suitable amount of energy (resonant with an electronic transition), a molecule goes to an excited state; this excitation is followed by a series of non-radiative vibrational relaxation processes that take the molecule to a lower energy vibrational level; then, a photon is emitted allowing the decay of the molecule back to its ground state (Fig. 3.1).

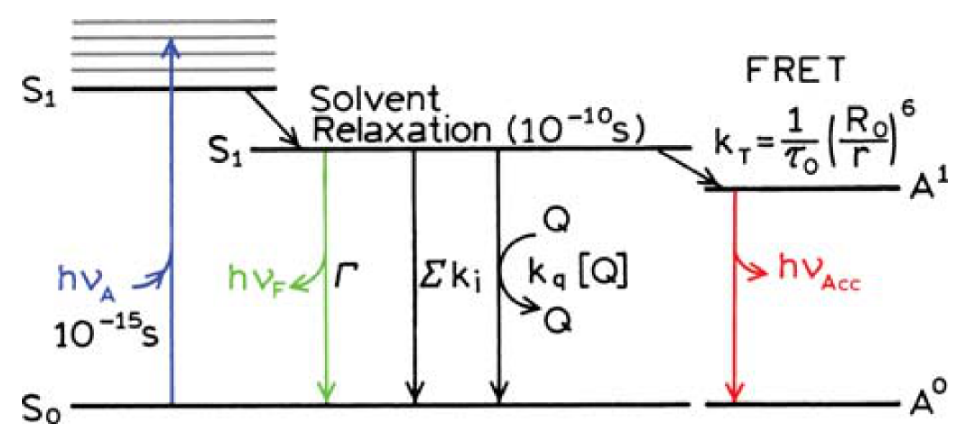

Figure 3.1 - This simplified Jablonski diagram pictures the fluorescence phenomenon: in this case, a molecule is excited from its ground state $S_{0}$ to a higher energy state by a photon of energy $h \nu_{A}$. A series of non-radiative vibrational relaxation processes take it to a lower vibrational energy level in $S_{1}$, where a fluorescence photon of energy $h \nu_{F}$ may be emitted allowing the molecule to return to $S_{0}$. Other processes may also allow the de-excitation of the molecule without the emission of a photon. The de-excitation of the molecule usually takes a few nanoseconds to occur.

Source: LAKOWICZ ${ }^{4}$

When there is a change in the spin multiplicity of the system (intersystem crossing) before the emission of the photon, the transition back to the ground state is not spin allowed and this decay takes much longer times. In this case, the phenomenon is called phosphorescence. Typically, fluorescence decays take a few nanoseconds to occur while phosphorescence demand milliseconds. In this work, we will focus solely on the fluorescence phenomenon. 
Common fluorescence related techniques are based on steady-state spectroscopy, which means that the measurements are made over the long term time average of the emitted light. Although steady-state techniques were and are successfully employed on many fields of research, important information can often be obtained from the study of the fluorescence temporal dynamics. The time-resolved spectroscopic techniques usually require a more complex instrumentation but can investigate much more intrinsic properties of the sample, being less affected by absorption and scattering on both the excitation and emission wavelengths. ${ }^{33}$ A brief overview on the fluorescence dynamics and on the time-resolved spectroscopy technique will be presented on the following sections.

\subsection{Temporal behavior of fluorescence}

For a sufficiently large number of molecules on the excited state, we can assume the population densities to be a continuum distribution instead of a more realistic Poisson distribution. In this case, in the absence of saturation and another nonlinear effects, it is reasonable to propose that the decay rate of such a system will be proportional to the number of molecules on the excited state. This way, the probabilistic temporal behavior of a set molecules initially at the excited state can be described by the rate equation

$$
\frac{d n(t)}{d t}=-\Gamma n(t)
$$

where $n(t)$ is the number of molecules in the excited state and $\Gamma$ the decay ratio. This equation has a exponential decay as its only solution. If a sample is instantaneously excited at $t=t_{0}$ by an impulse of the form

$$
P_{\text {exc }}(t)=E_{\text {pulse }} \delta\left(t-t_{0}\right)
$$

being $E_{\text {pulse }}$ the energy of the pulse, the fluorescence power decay $F(t)$ would follow the equation

$$
F(t)=A e^{-\Gamma t} H\left(t-t_{0}\right),
$$

where $A$ is a power amplitude parameter and $H\left(t-t_{0}\right)$ is the Heaviside or step function with edge at $t=t_{0}$. The fluorescence power function normalized by the pulse energy can be written as

$$
f(t)=a e^{-\Gamma t} H\left(t-t_{0}\right)
$$

where $a=A / E_{\text {pulse }}$. Now, it is convenient to arbitrarily choose $t_{0}=0$ for all further anal- 
ysis. The normalized fluorescence typical behavior using this assumption is illustrated on Fig. 3.2.

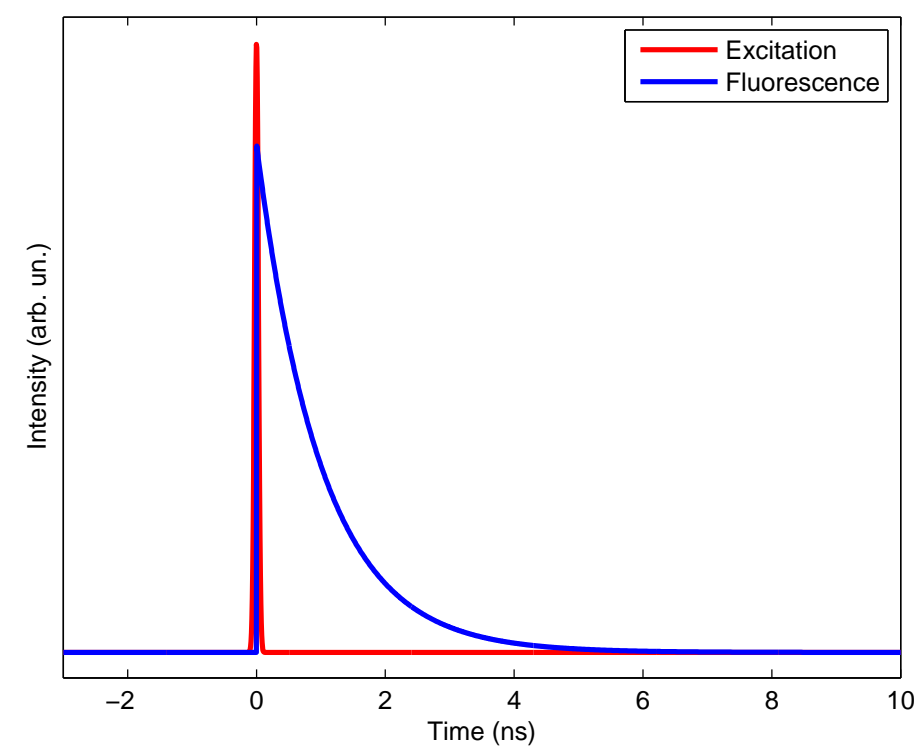

Figure 3.2 - When a fluorescent sample is instantaneously excited, the fluorescence will decay exponentially. In some cases, a multi-exponential behavior is observed.

Source: By the author.

The average lifetime $\tau$ can be defined as the average value of $t$ for the distribution $f(t)$, i.e.

$$
\tau=\langle t\rangle=\frac{\int_{-\infty}^{\infty} t f(t) d t}{\int_{-\infty}^{\infty} f(t) d t}
$$

For $f(t)$ as shown on equation 3.3, $\tau$ is calculated as follows:

$$
\tau=\frac{\int_{-\infty}^{\infty} t a e^{-\Gamma t} H(t) d t}{\int_{-\infty}^{\infty} a e^{-\Gamma t} H(t) d t}=\frac{\int_{0}^{\infty} t e^{-\Gamma t} d t}{\int_{0}^{\infty} e^{-\Gamma t} d t}=\frac{1}{\Gamma} .
$$

So, from the moment of the excitation on, the decay can be written as

$$
f(t)=a e^{-\frac{t}{\tau}}
$$

In practice, the fluorescence emission competes with some non-radiative process on the depopulation of the excited state. The total decay rate $\Gamma$ is written as

$$
\Gamma=k_{R}+k_{N R}
$$


where $k_{R}$ is the radiative decay rate and $k_{N R}$ is the non-radiative decay rate. We can write the lifetime of the excited state, or the fluorescence lifetime, as

$$
\tau=\frac{1}{k_{R}+k_{N R}}
$$

To relate those parameters ( $a$ and $\tau$ ) to what is measurable on the steady-state approach, we can calculate the fluorescence intensity upon continuous excitation by expressing the continuous excitation as a combination of infinite impulse functions as in the use of Green's functions. As the system is linear, the solution $Y(t)$ for the continuous and constant excitation $\left(P_{\text {exc }}(t)=P_{0}\right)$ is the sum of the solutions for each individual impulse function, so it can be written as follows:

$$
\begin{aligned}
Y(t) & =\int_{-\infty}^{\infty} f\left(t^{\prime}\right) P_{e x c}\left(t-t^{\prime}\right) d t^{\prime}=P_{0} \int_{-\infty}^{\infty} f\left(t^{\prime}\right) d t^{\prime} \\
& =P_{0} \int_{0}^{\infty} a e^{-t^{\prime} / \tau} d t^{\prime}=P_{0} a \tau .
\end{aligned}
$$

Therefore, the fluorescence steady-state intensity upon continuous excitation is constant as one could expect. Most importantly, the emission intensity depends on the product of $a$ and $\tau$.

This result shows explicitly that changes in the amplitude parameter $a$ cannot be directly translated to proportional changes in the fluorescence intensity and vice versa. Fluorescence intensity amplitude must be differentiated from the integrated fluorescence intensity and as it is a completely different contrast parameter.

Different fluorophores may present different lifetimes and even the same type of fluorophore can present significant variations in its fluorescence lifetime due to the modifications on its microenvironment. In the case $N$ molecules are simultaneously emitting fluorescence with different decay rates, the fluorescence decay must be written as a combination of $N$ exponential decays as follows:

$$
f(t)=\sum_{i=1}^{N} a_{i} e^{-\frac{t}{\tau_{i}}} .
$$

In this case, $a_{i}$ represents the amplitudes of the individual exponential decays with lifetimes of $\tau_{i}$ composing the function $f(t)$, with $i=1, \ldots, N$.

For such multi-exponential decays, the average lifetime $\tau_{\text {avg }}$ can be calculated through the equation 3.4 : 


$$
\begin{aligned}
\tau_{\text {avg }}=\frac{\int_{0}^{\infty}\left(\sum_{i=1}^{N} t a_{i} e^{-t / \tau_{i}}\right) d t}{\int_{0}^{\infty}\left(\sum_{i=1}^{N} a_{i} e^{-t / \tau_{i}}\right) d t}=\frac{\sum_{i=1}^{N}\left(\int_{0}^{\infty} t a_{i} e^{-t / \tau_{i}} d t\right)}{\sum_{i=1}^{N}\left(\int_{0}^{\infty} a_{i} e^{-t / \tau_{i}} d t\right)} \\
\tau_{\text {avg }}=\frac{\sum_{i=1}^{N} a_{i} \tau_{i}^{2}}{\sum_{i=1}^{N} a_{i} \tau_{i}} .
\end{aligned}
$$

\subsection{Time-resolved fluorescence spectroscopy}

The time-resolved fluorescence spectroscopy techniques can be divided in two groups: the time-domain and the frequency-domain techniques. The former includes different ways to record or reconstruct the fluorescence decay over time after excitation through a short light pulse while the latter uses a periodic illumination and collects information like average intensity and phase shift between illumination and fluorescence to access information about the fluorophores fluorescence lifetime. In the present work we focus only on the time-domain measurements, but a detailed discussion about the frequencydomain has been done by Lakowicz. ${ }^{4}$

The most commonly used time-resolved time-domain fluorescence spectroscopy technique is the TCSPC. In this technique, a laser with short pulses (typically few nanoseconds or some picoseconds) is used to excite the sample and the fluorescence is collected by a high sensitivity fast detector such as avalanche photodiodes or some photomultiplier tubes. Instead of recording directly the decay, the experiment conditions are such that no more than one photon is collected by laser pulse. The delay between pulse and photon detection is measured during multiple pulses and, after a sufficient number of photons is detected, a histogram of those delays can be constructed. This histogram is, essentially, the fluorescence decay curve. ${ }^{4,34,35}$ The working principle of a TCSPC is illustrated on Fig. 3.3.

While the TCSPC modules usually have a better time resolution, signal-to-noise ratio and lower cost compared to the other available time-domain options, ${ }^{34-36}$ they require the acquisition during multiple pulses to obtain a good fluorescence decay curve. This makes the process slower than the method of directly acquiring the measurement with a fast detector and digitizer, what can be a problem for in vivo imaging, where a short acquisition time is necessary to avoid or reduce tissue motion artifact. 


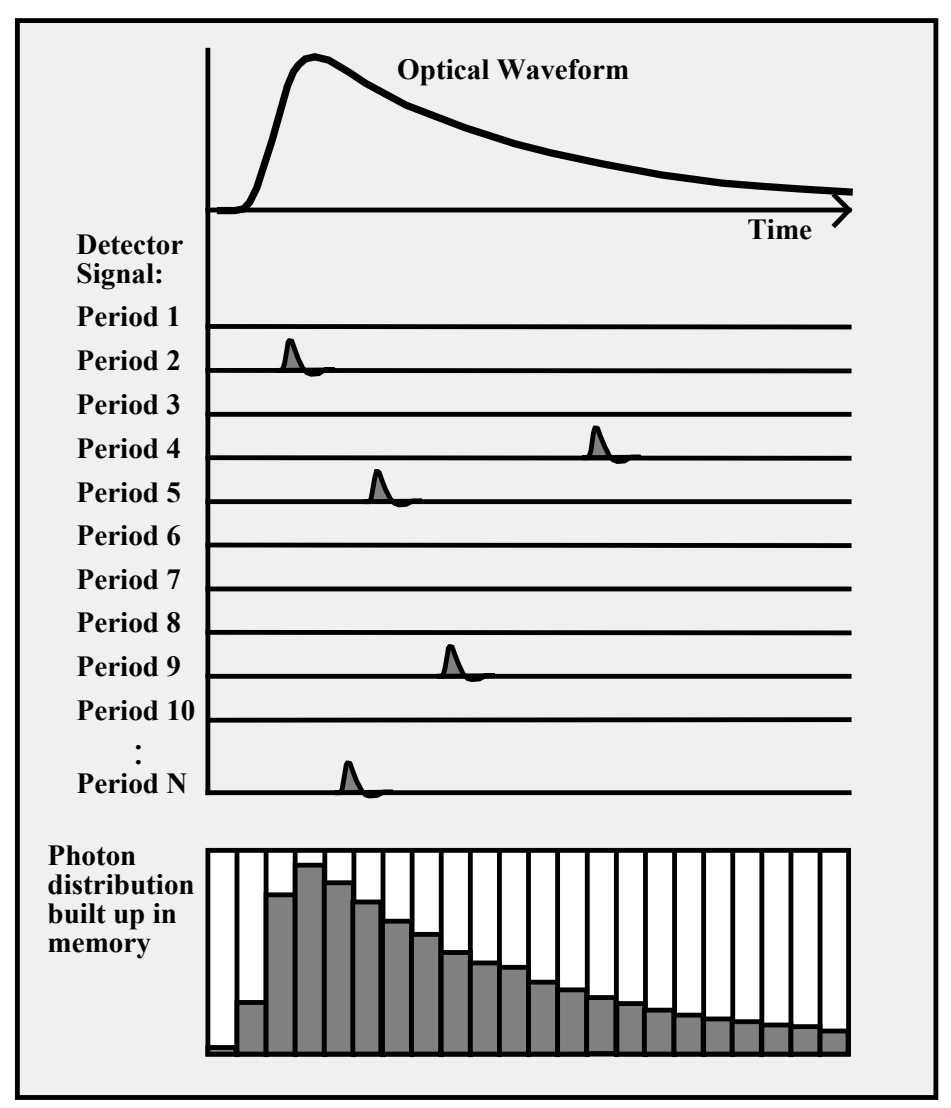

Figure 3.3 - Working principle of a TCSPC measurement: the configuration of the experiment is such that no more than one photon is collected by laser pulse. After a sufficiently high number of photons is collected, a histogram of the delay between laser pulse and fluorescence photon is constructed. This histogram is the fluorescence decay curve.

Source: BECKER ${ }^{36}$

In this method, the fluorescence is collected by a high-speed and high-sensitivity detector, i.e. a MCP-PMT, connected to a broadband amplifier and a fast digitizer. At a rate specified by the digitizer sampling rate, a measurement is made and the intensity value is recorded. Compared to the TCSPC, this method requires a much faster detector and a much broader electronic bandwidth, making the system more expensive but allowing the acquisition of good quality fluorescence decays with a single laser pulse. ${ }^{23}$

For any time-resolved spectroscopy technique used, the measurement system response is not immediate. Also, the laser pulse, despite being short, has a nonzero width. If a fluorescence sample had, hypothetically, a instantaneous fluorescence decay $(\tau \rightarrow 0)$, the measured fluorescence signal would not be an instantaneous impulse, but a spread function that would represent a response function of the equipment. This function is called Instrument Response Function (IRF).

Any fluorescence decay measured will be the convolution of the IRF with the sample impulse response curve $f(t)$ described on equation 3.10. This can be written as: 


$$
y(t)=f(t) * g(t)=\int_{-\infty}^{\infty} f\left(t^{\prime}\right) u\left(t-t^{\prime}\right) d t^{\prime}
$$

where $y(t)$ is the measured signal, $f(t)$ is the impulse response fluorescence decay defined on equation 3.10 and $u(t)$ is the IRF, which can be directly measured by recording the temporal distribution of the laser pulse scattering.

As result, the measured data will have a smoother shape than the curve shown on Fig. 3.2, as pictured on Fig. 3.4.

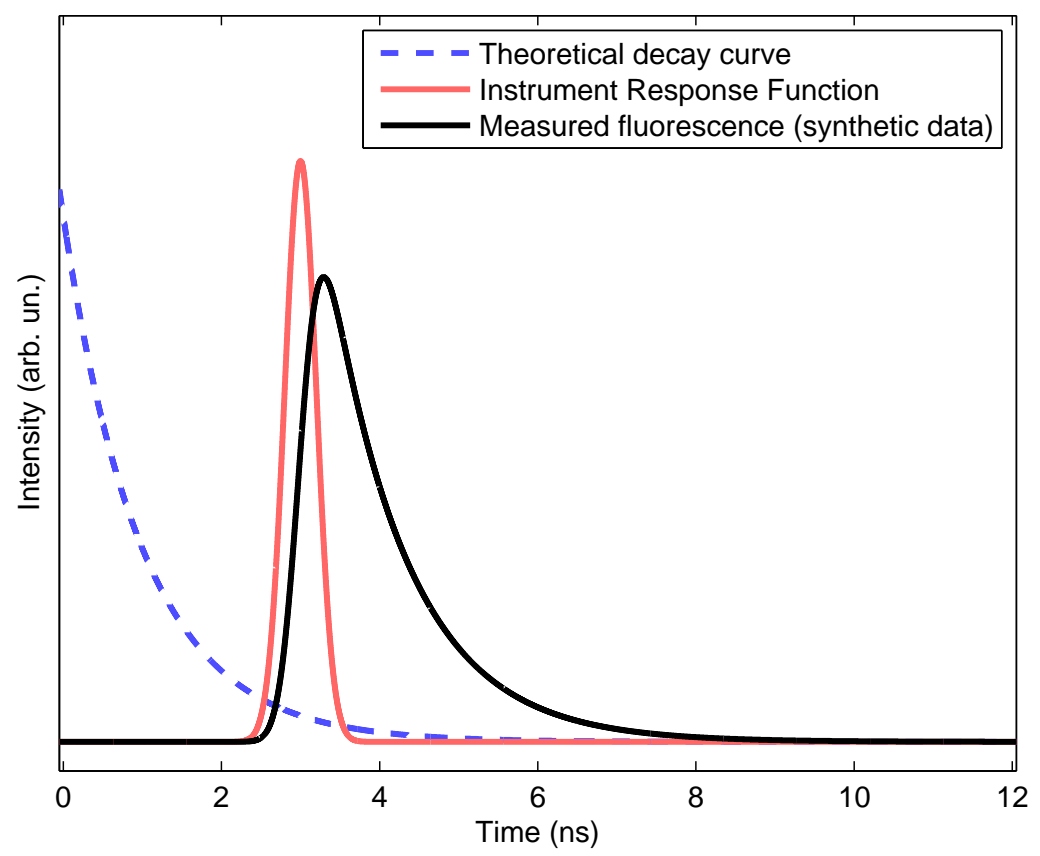

Figure 3.4 - The measured signal is the convolution of the fluorescence decay curve with the Instrument Response Function.

Source: By the author.

Conveniently, the convolution theorem ${ }^{37}$ states that the Fourier transform of a convolution between two functions equals to the product of the Fourier Transform of the individual functions. So, we can rewrite the equation 3.12 in the frequency space as

$$
\mathcal{F}\{y(t)\}(\omega)=\mathcal{F}\{f(t)\}(\omega) \cdot \mathcal{F}\{u(t)\}(\omega),
$$

where $\mathcal{F}\{g(t)\}(\omega)$ denotes the Fourier Transform of a function $g(t)$. We can use the more compact notation:

$$
\tilde{y}=\tilde{f} \cdot \tilde{u}
$$

where $\tilde{g}=\tilde{g}(\omega)=\mathcal{F}\{g(t)\}(\omega)$. 
All those functions are, in reality, measured by a digitizer with a specified acquisition rate $F_{S}=1 / T_{S}$, where $T_{S}$ is the time between consecutive time samples. Therefore, they must be represented in a discrete time basis. For this, instead of specifying these functions as functions of time, we will represent them as vectors indexed by a time index $[l]$. The actual time related to the indexes $[l]$ forms a linearly spaced vector, with

$$
t[l]=l \cdot T_{S}
$$

with $l=0, \ldots, L-1$, being $L$ the number of time points.

In the discrete time basis description, the convolution between the fluorescence impulse vector $\mathbf{f}$, and the IRF vector $\mathbf{u}$, analogously to the equation 3.12 , can be written as:

$$
y[l]=\sum_{j=0}^{l} f[l] \cdot u[l-j] .
$$

We can now explicitly calculate each element of the vector $\mathbf{y}$ :

$$
\mathbf{y}=\left[\begin{array}{c}
y[0] \\
y[1] \\
\vdots \\
y[L-1]
\end{array}\right]=\left[\begin{array}{c}
u[0] \cdot f[0] \\
u[1] \cdot f[0]+u[0] \cdot f[1] \\
\vdots \\
u[L-1] \cdot f[0]+\cdots+u[0] \cdot f[L-1]
\end{array}\right]
$$

It is possible to see that this equation can be conveniently written as

$$
\left[\begin{array}{c}
y[0] \\
y[1] \\
\vdots \\
y[L-1]
\end{array}\right]=\left[\begin{array}{cccc}
u[0] & 0 & \ldots & 0 \\
u[1] & u[0] & \ldots & 0 \\
\vdots & \vdots & \ddots & \vdots \\
u[L-1] & u[L-2] & \ldots & u[0]
\end{array}\right]\left[\begin{array}{c}
f[0] \\
f[1] \\
\vdots \\
f[L-1]
\end{array}\right]
$$

or in a more compact way as

$$
\mathbf{y}=U \mathbf{f}
$$

with

$$
U=\left[\begin{array}{cccc}
u[0] & 0 & \ldots & 0 \\
u[1] & u[0] & \ldots & 0 \\
\vdots & \vdots & \ddots & \vdots \\
u[L-1] & u[L-2] & \ldots & u[0]
\end{array}\right]
$$

The matrix $U$ is special kind of diagonal-constant matrix called Toeplitz matrix. It 
is convenient, especially for some parts of the discussion that will follow, to write the convolution operation in this form.

At this point, it is also convenient to call back the convolution theorem, which is still valid for the discrete time basis, and can be efficiently used to numerically compute the convolution between vectors thanks to extremely optimized Discrete Fourier Transform algorithms, namely Fast Fourier Transform Algorithms. Applying the Inverse Fourier Transform to the equation 3.13, in the discrete time basis description, we can calculate y as follows:

$$
\mathbf{y}=\mathrm{iDTFT}[\operatorname{DTFT}(\mathbf{f}) \cdot \operatorname{DTFT}(\mathbf{u})]
$$

with DTFT and iDTFT denoting the Discrete Time Fourier Transform and its inverse transformation, and $\mathbf{a} \cdot \mathbf{b}$ representing the pointwise product between same sized vectors $\mathbf{a}$ and $\mathbf{b}$.

Now that we have covered some observation models for the convolution of the fluorescence signal, the next step is to review some methods to perform the lifetime estimations and deconvolution of the IRF from the measured signal.

\subsection{Lifetime estimation and fluorescence signal deconvolution}

The equation 3.18 relates $\mathbf{y}, \mathbf{f}$, and $\mathbf{u}$ through an analytic expression. Although this equation can be inverted so that we can isolate $\mathbf{f}$ as

$$
\mathbf{f}=\operatorname{iDTFT}[\operatorname{DTFT}(\mathbf{y}) \cdot / \operatorname{DTFT}(\mathbf{u})]
$$

where $\mathbf{a} . / \mathbf{b}$ represents the pointwise division operation between same sized vectors a and $\mathbf{b}$, this equation is of no practical in our case because the Power Spectral Density (PSD) of the IRF, where

$$
\operatorname{PSD}(\mathbf{u})=|\operatorname{DTFT}(\mathbf{u})|^{2}
$$

has a finite bandwidth for typical IRFs, which are fairly similar to Gaussian functions. This means that, with a finite bandwidth, the IRF convolution completely filters the higher frequencies components from the signal. Those higher frequencies components are lost and cannot be recovered by the applying the equation 3.19.

As the typical IRFs are fairly similar to Gaussian functions, its bandwidth will be close to the lower limit imposed by of the order of the Gabor $\operatorname{limit}^{38,39}$ (analogous to the Heisenberg's Uncertainty Principle for the signal processing context), which means: 


$$
\sigma_{\omega} \approx \frac{1}{2 \sigma_{t}}
$$

where $\sigma_{\omega}$ is the bandwidth of the IRF and $\sigma_{t}$ is the time width of the IRF.

Additionally, both $\mathbf{y}$ and $\mathbf{u}$ are measured vectors and have noise. The Gaussian noise amplitude does not depend on the signal intensity and its frequency components extend up to Nyquist limit, so beyond the bandwidth of $\mathbf{u}$. As we try to perform the deconvolution using the equation 3.19, the division operation results in noise amplification, especially for higher frequencies, where the power spectrum density of the IRF comes closer to zero. This results in a rather noisy result where the expected features of the curve are barely observable.

We believe the noise amplification by the Fourier transform-based deconvolution can be reduced with the use of digital filters, but further filtering would probably make worse the elimination of higher frequencies components of the impulse response signal.

Another seemingly straightforward way to perform the deconvolution is by inverting the linear equation 3.16. It may seem that this equation can be easily solved for $\mathbf{f}$ as

$$
\mathbf{f}=U^{-1} \mathbf{y}
$$

However, the determinant of the Toeplitz matrix $U$ is zero, which means that $U$ is singular for any generating IRF vector $\mathbf{u}$. This means that rows of $U$ are linear dependent, so $U$ is a short ranked matrix and, therefore, the system of linear equations displayed in equation 3.16 does not have a single solution, i.e. there is not a single $\mathbf{f}$ that generates y.

Both the Fourier transform based approach and the Toeplitz matrix convolution lead to problems to perform the analytical deconvolution of the signal. In fact, the deconvolution of time-resolved fluorescence spectroscopy data need more complex methods which we will discuss further on the subsection 3.4.2.

However, there are simpler and faster ways of performing the average lifetime estimations of raw signals that are very useful for quick assessment of lifetime changes and for real time previewing of the data. We will discuss some of those methods in the next subsection.

\subsubsection{Fast lifetime estimation from raw signal}

A common method for fast lifetime estimation is often called the Center of Mass Method. This method is based on the direct application of the equation 3.4. This equation holds true for the impulse response fluorescence function $f(t)$, i.e. the deconvoluted 
signal. To avoid the problem of needing to deconvolute the IRF from the measured signal, the signal is discarded for every point before its maximum, and the time vector is recalculated so this new cropped measured signal vector starts at $t=0$. The reasoning behind this approximation is that if the IRF is much narrower than the average lifetime, the decaying part of the measured signal will be approximately equal to $f(t)$.

In the discrete time basis, we write this approximation this equation as:

$$
\tau_{\text {avg }}=\frac{\sum_{l=l_{\max }}^{L-1}\left(t[l]-t\left[l_{\max }\right]\right) \cdot y[l]}{\sum_{l=l_{\max }}^{L-1} y[l]},
$$

where $l_{\max }$ is the time index of maximum intensity of the measured signal, which means

$$
y\left[l_{\text {max }}\right]=\max (y)
$$

This method is very sensitive to noise and highly depends on the IRF being much narrower than the fluorescence impulse response to provide accurate results of the average lifetime, but even in cases where the absolute lifetime values are not accurate, it is possible to detect lifetime modifications.

Another important method for the fast analysis of the fluorescence lifetime data is based on the phasor approach. ${ }^{40}$ In this method, proposed by Digman et al., the Fourier Transforms of the measured signal is calculated and a phasor map is generated. While this method was shown to succeed in providing lifetime sensitive contrast in biological samples, it does not provide any correction for the IRF convolution nor does it focus in providing absolute fluorescence lifetime values. Here, we propose a slightly modified method based on the phasor approach to address those points.

We argued that the equation 3.19 is not of useful for the deconvolution of measured signal because the measured signal has had its higher frequencies filtered off by the IRF and because of the noise amplification in higher frequencies.

If we assume a fluorescence impulse response function $f(t)$ as a exponential decay as the equation 3.3, we can analytically calculate its Fourier Transform:

$$
\begin{aligned}
\tilde{f}(\omega) & =\frac{a}{\Gamma+i \omega}=\frac{a}{1 / \tau+i \omega} \\
& =\frac{a / \tau}{1 / \tau^{2}-\omega^{2}}+i \frac{a \omega}{1 / \tau^{2}-\omega^{2}}
\end{aligned}
$$


It is easy to verify that we can now calculate $\tau$ as

$$
\tau=\frac{1}{\omega} \frac{\operatorname{Imag}\{\tilde{f}(\omega)\}}{\operatorname{Real}\{\tilde{f}(\omega)\}},
$$

where Imag and Real represent the imaginary and real parts of their arguments. The difference between this approach and the direct application of the equation 3.19, is that in this case, the equation is valid for any angular frequency $\omega$. So we can choose one single $\omega$ and evaluate $\tau$, while the evaluation of the equation 3.19 is done with a integration over all values of $\omega$.

We can evaluate $\tau$ using the equation 3.23 at low values of $\omega$, to avoid the problems related to the higher frequencies. We choose the lowest nonzero angular frequency $\omega_{0}$, where the Signal-to-Noise Ratio (SNR) and the signal PSD are maximum:

$$
\omega_{0}=2 \pi \frac{F_{S}}{L}
$$

being $F_{S}$ the sampling frequency or sampling rate, and $L$ the number of time points. The angular frequency vector, as the time vector, is an evenly spaced vector by steps of size equal to $\omega_{0}$. We will call the frequency index $[\tilde{l}]$ of the lowest nonzero frequency $\left[\tilde{l_{0}}\right]$, so $\omega_{0}=\omega\left[\tilde{l_{0}}\right]$.

In the discrete time basis representation, the equation 3.23 evaluated at $\omega=\omega_{0}$ becomes:

$$
\tau=\frac{1}{\omega_{0}} \frac{\operatorname{Imag}\left\{\tilde{y}\left[\tilde{l}_{0}\right] \cdot / \tilde{u}\left[\tilde{l}_{0}\right]\right\}}{\operatorname{Real}\left\{\tilde{y}\left[\tilde{l_{0}}\right] \cdot / \tilde{u}\left[\tilde{l_{0}}\right]\right\}}
$$

where

$$
\begin{aligned}
& \tilde{\mathbf{y}}=\operatorname{DTFT}(\mathbf{y}), \\
& \tilde{\mathbf{u}}=\operatorname{DTfT}(\mathbf{u}) .
\end{aligned}
$$

This approach also has some limitations. One of the main problems with it is that it is based on the analytical solution of $\tau$ from the equation 3.22 for one $\omega$, and this is only possible for a single exponential decay. This problem is common to the fast lifetime estimations methods presented here: both fail to provide information about all the parameters of multiple exponential decays. To have this information, the analysis by more complex deconvolution methods are necessary. Some deconvolution methods are discussed in the next subsection. 


\subsubsection{Deconvolution methods}

As previously discussed, the Toeplitz matrix $U$ is singular and therefore there is no single solution for $\mathbf{f}$ on the equation 3.16. So, in order to perform the deconvolution, we must start with some knowledge of the general shape or of some conditions of the impulse response fluorescence vector $\mathbf{f}$. One way to do that is to expand the function $f(t)$ that generates the vector $\mathbf{f}$ as a linear combination of basis functions $b_{n}(t)$. In the discrete time basis notation, this can be written as

$$
f[l]=\sum_{n=0}^{N-1} c_{n} b_{n}[l]
$$

where $N$ is the number of functions in the basis and $c_{n}$ is the $n^{\text {th }}$ weighting coefficient.

For multiexponential decays, it is reasonable to choose a function basis composed of exponential decays:

$$
b_{n}\left(t ; \tau_{n}\right)=\exp \left(-\frac{t}{\tau_{n}}\right)
$$

where each basis function $b_{n}$ is a function of an external parameter $\tau_{n}$. In the discrete time basis, we can write the basis vectors as

$$
\mathbf{b}_{\mathbf{n}}\left(\tau_{n}\right)=\exp \left(-\frac{\mathbf{t}}{\tau_{n}}\right)
$$

and each one of its elements as

$$
b_{n}[l]\left(\tau_{n}\right)=\exp \left(-\frac{t[l]}{\tau_{n}}\right)
$$

for $n=0, \ldots, N-1$.

We can now explicitly write all elements of equation 3.25 in matrix form:

$$
\left[\begin{array}{c}
f[0] \\
f[1] \\
\vdots \\
f[L-1]
\end{array}\right]=\left[\begin{array}{cccc}
b_{1}[0]\left(\tau_{1}\right) & b_{2}[0]\left(\tau_{2}\right) & \ldots & b_{N}[0]\left(\tau_{N}\right) \\
b_{1}[1]\left(\tau_{1}\right) & b_{2}[1]\left(\tau_{2}\right) & \ldots & b_{N}[1]\left(\tau_{N}\right) \\
\vdots & \vdots & \ddots & \vdots \\
b_{1}[L-1]\left(\tau_{1}\right) & b_{2}[L-1]\left(\tau_{2}\right) & \ldots & b_{N}[L-1]\left(\tau_{N}\right)
\end{array}\right]\left[\begin{array}{c}
c_{0} \\
c_{1} \\
\vdots \\
c_{N}
\end{array}\right]
$$

By no means the matrix of basis vectors elements needs to be square. The number $N$ of functions composing the basis can be chosen independently of the number $L$ of time points. We can also write this equation in a more compact way as: 


$$
\mathbf{f}=B \mathbf{c}
$$

where $B=B\left(\tau_{0}, \ldots, \tau_{N-1}\right)$ is the matrix of basis vectors. Substituting this result on equation 3.16, we have:

$$
\mathbf{y}=U B \mathbf{c} .
$$

With this equation, we can calculate $\mathbf{y}$ as a function of the weighting coefficients $\mathbf{c}$ and $\tau_{0}, \ldots, \tau_{N-1}$. Together, the weighting coefficients and individual lifetimes form the set of parameters $\mathbf{p}$.

$$
\mathbf{p}=\left\{c_{0}, \ldots, c_{N-1} ; \tau_{0}, \ldots, \tau_{N-1}\right\}
$$

The goal of this analysis, called the method of least squares, ${ }^{41,42}$ is to find the combination of those parameters that generate the $\mathbf{y}$ that better matches the vector of measured data $\hat{\mathbf{y}}$. This means our goal is to find the set of parameters that minimize the sum of squared residuals $\xi$, with

$$
\xi=\sum_{l=0}^{L-1}(\hat{y}[l]-y[l])^{2} .
$$

We can rewrite this equation as

$$
\begin{aligned}
\xi(\mathbf{p}) & =(\hat{\mathbf{y}}-\mathbf{y}(\mathbf{p}))^{\top}(\hat{\mathbf{y}}-\mathbf{y}(\mathbf{p})) \\
& =\hat{\mathbf{y}}^{\top} \hat{\mathbf{y}}-\hat{\mathbf{y}}^{\top} \mathbf{y}-\mathbf{y}^{\top} \hat{\mathbf{y}}+\mathbf{y}^{\top} \mathbf{y} \\
& =\hat{\mathbf{y}}^{\top} \hat{\mathbf{y}}-2 \hat{\mathbf{y}}^{\top} \mathbf{y}+\mathbf{y}^{\top} \mathbf{y}
\end{aligned}
$$

where $\mathbf{v}^{\top}$ denotes the transposed vector $\mathbf{v}$.

We now consider a perturbation $\mathbf{h}$ to the set of parameters $\mathbf{p}$ and expand $\mathbf{y}(\mathbf{p}+\mathbf{h})$ up to the first-order element of the Taylor series:

$$
\begin{aligned}
\mathbf{y}(\mathbf{p}+\mathbf{h}) & =\mathbf{y}(\mathbf{p})+\left[\frac{\partial \mathbf{y}}{\partial \mathbf{p}}\right] \mathbf{h} \\
& =\mathbf{y}(\mathbf{p})+J \mathbf{h}
\end{aligned}
$$

where 


$$
J=\left[\begin{array}{cccc}
\frac{\partial y[0]}{\partial p[0]} & \frac{\partial y[0]}{\partial p[1]} & \cdots & \frac{\partial y[0]}{\partial p[N-1]} \\
\frac{\partial y[1]}{\partial p[0]} & \frac{\partial y[1]}{\partial p[1]} & \cdots & \frac{\partial y[1]}{\partial p[N-1]} \\
\vdots & \vdots & \ddots & \vdots \\
\frac{\partial y[L-1]}{\partial p[0]} & \frac{\partial y[L-1]}{\partial p[1]} & \cdots & \frac{\partial y[L-1]}{\partial p[N-1]}
\end{array}\right]
$$

is the $L \times N$ Jacobian matrix. We can now substitute the equation 3.34 on the equation 3.33 to evaluate $\xi(\mathbf{p}+\mathbf{h})$ :

$$
\begin{aligned}
\xi(\mathbf{p}+\mathbf{h}) & =\hat{\mathbf{y}}^{\top} \hat{\mathbf{y}}-\hat{\mathbf{y}}^{\top} \mathbf{y}(\mathbf{p}+\mathbf{h})+\mathbf{y}(\mathbf{p}+\mathbf{h})^{\top} \mathbf{y}(\mathbf{p}+\mathbf{h}) \\
& =\hat{\mathbf{y}}^{\top} \hat{\mathbf{y}}-2 \hat{\mathbf{y}}^{\top}[\mathbf{y}(\mathbf{p})+J \mathbf{h}]+[\mathbf{y}(\mathbf{p})+J \mathbf{h}]^{\top}[\mathbf{y}(\mathbf{p})+J \mathbf{h}] \\
& =\hat{\mathbf{y}}^{\top} \hat{\mathbf{y}}-2 \hat{\mathbf{y}}^{\top} \mathbf{y}-2 \hat{\mathbf{y}}^{\top} J \mathbf{h}+\mathbf{y}^{\top} \mathbf{y}+\mathbf{y}^{\top} J \mathbf{h}+(J \mathbf{h})^{\top} \mathbf{y}+(J \mathbf{h})^{\top} J \mathbf{h} \\
& =\hat{\mathbf{y}}^{\top} \hat{\mathbf{y}}-2 \hat{\mathbf{y}}^{\top} \mathbf{y}-2 \hat{\mathbf{y}}^{\top} J \mathbf{h}+\mathbf{y}^{\top} \mathbf{y}+\mathbf{y}^{\top} J \mathbf{h}+\mathbf{h}^{\top} J^{\top} \mathbf{y}+\mathbf{h}^{\top} J^{\top} J \mathbf{h} \\
& =\hat{\mathbf{y}}^{\top} \hat{\mathbf{y}}-2 \hat{\mathbf{y}}^{\top} \mathbf{y}-2 \hat{\mathbf{y}}^{\top} J \mathbf{h}+\mathbf{y}^{\top} \mathbf{y}+2 \mathbf{y}^{\top} J \mathbf{h}+\mathbf{h}^{\top} J^{\top} J \mathbf{h} \\
& =\hat{\mathbf{y}}^{\top} \hat{\mathbf{y}}-2 \hat{\mathbf{y}}^{\top} \mathbf{y}-2\left(\hat{\mathbf{y}}^{\top}-\mathbf{y}^{\top}\right) J \mathbf{h}+\mathbf{y}^{\top} \mathbf{y}+\mathbf{h}^{\top} J^{\top} J \mathbf{h} \\
& =\hat{\mathbf{y}}^{\top} \hat{\mathbf{y}}-2 \hat{\mathbf{y}}^{\top} \mathbf{y}+\mathbf{y}^{\top} \mathbf{y}-2(\hat{\mathbf{y}}-\mathbf{y})^{\top} J \mathbf{h}+\mathbf{h}^{\top} J^{\top} J \mathbf{h}
\end{aligned}
$$

The perturbation $\mathbf{h}$ that minimizes $\xi$ can be calculated by finding

$$
\frac{\partial}{\partial \mathbf{h}} \xi(\mathbf{p}+\mathbf{h}) \equiv \nabla_{\mathbf{h}} \xi(\mathbf{p}+\mathbf{h})=0
$$

We can calculate this gradient directly from equation 3.36, which results in the vector:

$$
\begin{aligned}
\frac{\partial}{\partial \mathbf{h}} \xi(\mathbf{p}+\mathbf{h}) & =-2(\hat{\mathbf{y}}-\mathbf{y})^{\top} J+\frac{\partial}{\partial \mathbf{h}}\left(\mathbf{h}^{\top} J^{\top} J \mathbf{h}\right) \\
& =-2(\hat{\mathbf{y}}-\mathbf{y})^{\top} J+\left[\frac{\partial}{\partial \mathbf{h}}\left(\mathbf{h}^{\top} J^{\top} J \mathbf{k}\right)\right]_{\mathbf{k}=\mathbf{h}}+\left[\frac{\partial}{\partial \mathbf{h}}\left(\mathbf{k}^{\top} J^{\top} J \mathbf{h}\right)\right]_{\mathbf{k}=\mathbf{h}} \\
& =-2(\hat{\mathbf{y}}-\mathbf{y})^{\top} J+\left[\frac{\partial}{\partial \mathbf{h}}\left(\mathbf{k}^{\top} J^{\top} J \mathbf{h}\right)\right]_{\mathbf{k}=\mathbf{h}}+\left[\mathbf{k}^{\top} J^{\top} J\right]_{\mathbf{k}=\mathbf{h}} \\
& =-2(\hat{\mathbf{y}}-\mathbf{y})^{\top} J+\left[\mathbf{k}^{\top} J^{\top} J\right]_{\mathbf{k}=\mathbf{h}}+\mathbf{h}^{\top} J^{\top} J \\
& =-2(\hat{\mathbf{y}}-\mathbf{y})^{\top} J+\mathbf{h}^{\top} J^{\top} J+\mathbf{h}^{\top} J^{\top} J \\
& =-2(\hat{\mathbf{y}}-\mathbf{y})^{\top} J+2 \mathbf{h}^{\top} J^{\top} J .
\end{aligned}
$$


Using the equations 3.37 and 3.38, we have:

$$
\mathbf{h}_{\mathrm{gn}}{ }^{\top} J^{\top} J=(\hat{\mathbf{y}}-\mathbf{y})^{\top} J
$$

Transposing this equation and solving for $\mathbf{h}_{\mathbf{g n}}$, we get:

$$
\mathbf{h}_{\mathrm{gn}}=\left(J^{\top} J\right)^{-1} J^{\top}(\hat{\mathbf{y}}-\mathbf{y})
$$

So we know the perturbation $\mathbf{h}_{\mathbf{g n}}$ would minimize $\xi$ within our first-order Taylor series approximation. By iteratively updating the value of $\mathbf{p}$ by a shift of $\mathbf{h}_{\mathbf{g n}}$, the set of parameters $\mathbf{p}$ will typically converge to the value that produces the smallest sum of squared residuals. This method, called Gauss-Newton method, allows us to iteratively find a set of parameters $\mathbf{p}$ that provide the best fit to the measured data. ${ }^{43}$

Several other non-iterative methods were developed to improve the analysis of timedomain fluorescence lifetime spectroscopy during the past years, including gating methods to improve the accuracy of the Rapid Lifetime Determination, ${ }^{44}$ the Integration for Extraction Method ${ }^{45,46}$ and the Phasor Approach. ${ }^{40,47}$ Also, field programmable gate arrays (FPGAs) with hardware-only, center of mass based, ${ }^{48,49}$ fast algorithms were implemented to speed up FLIM data analysis. ${ }^{50}$ Additionally, by expanding the fluorescence impulse response on the discrete time Laguerre polynomials basis, ${ }^{51}$ Jo et al. managed to perform a high quality, fast deconvolution of time-resolved spectroscopy data. $^{52}$

Now that we covered technical details regarding fluorescence lifetime spectroscopy and fluorescence lifetime spectroscopy data analysis, the next step would be to apply those concepts of time-resolved spectroscopy to imaging. The acquisition of a fluorescence decay curve for each point of a sample allows the construction of fluorescence lifetime microscopic maps. This imaging technique is discussed on the section 3.5.

\subsection{Fluorescence Lifetime Imaging Microscopy}

By the end of the 20th century, both the computational power and electronics speed requirements were finally achieved, allowing the idea of the time-resolved fluorescence spectroscopy to be applied on imaging. ${ }^{8}$ FLIM allows image contrast to be based both on fluorescence intensity and on fluorescence lifetime parameters. This concept is illustrated on Fig. 3.5, where a sample could have two different regions that cannot be distinguished by the fluorescence intensity. The lifetime maps, however, can clearly distinguish both regions. Such a behavior can be expected, for example, when there are regions with different ionic concentration on a sample, which may affect some fluorophores lifetimes. ${ }^{4}$ 


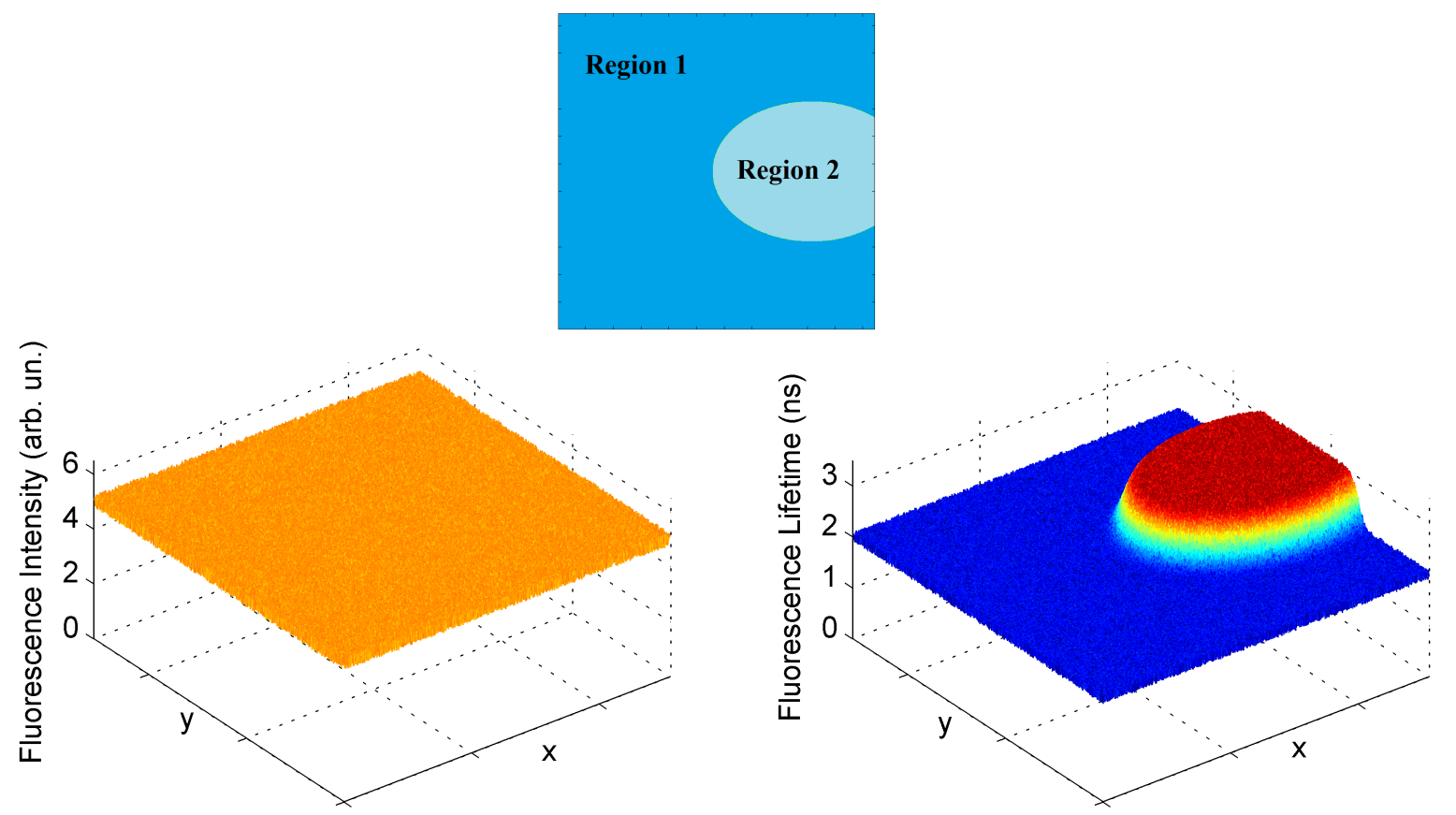

Figure 3.5 - Illustration of the concepts of FLIM: both a fluorescence intensity and lifetime maps can be produced. In some cases, the contrast produced by the fluorescence intensity can be not enough to distinguish different regions of a sample. In some cases those regions can be distinguished on the fluorescence lifetime map. (Figure generated with synthetic data.)

Source: By the author.

Another factor that can produce lifetime changes in a sample is the internal quenching, produced either by internal molecular rotation, excited state charge transfers or the existence of intersystem crossing. There are also external quenching mechanisms. The main ones are the Förster Resonance Energy Transfer, the Dexter Electron Transfer and the Dynamic Quenching. ${ }^{4,8}$

The phenomena that produces changes in lifetimes usually do so by increasing the non-radiative decay rate $k_{N R}$, which reduces the fluorescence lifetime as shown on Equation 3.8.

Considering FLIM application on biological tissues, the main indicated advantage is that this technique usually does not require the use of specific labeling exogenous fluorophores. The major target endogenous fluorophores are NADH and FAD, relevant electron transporters of the cellular respiratory chain. The fluorescence lifetimes of NADH and FAD provide a readout of the metabolic status of the interrogated tissue, which is an important information for the diagnosis of pathological conditions.

Each FLIM image ${ }^{\dagger}$ is composed of a significant amount of data. Modern FLIM systems operating in real time acquisition can produce data that easily exceeds tens

${ }^{\dagger}$ We call a FLIM dataset as an image for simplicity, but each data set consists of fluorescence decay curves for each pixel. 
of gigabit per second ${ }^{53,54}$ and this creates difficulties on data handling and, especially, data analysis. As FLIM raw data is ultimately not useful without processing, there is a bottleneck involving its treatment for real time

The analysis of each individual decay can be done as explained on subsection 3.4.2. However, the direct fitting of the exponential decays lead to very precise results, but this usually has high computational costs. Typically, a few milliseconds are required to perform the nonlinear least square analysis of a single fluorescence decay. For entire images the processing may take a few minutes even for modern computers using highly optimized algorithms.

In the next chapter, we will present the setup of FLIM system we assembled, some details regarding its control and signal acquisition, the handheld probe simulations and design, and the data analysis methods we used. We will also present a simplified setup used for preliminary testing and the mobile platform developed to allow the transportation of the system. Finally, we discuss the validation methods we will use and the protocol for the clinical study. 


\section{Material and methods}

In this chapter, we will present the setup of the built FLIM system, discussing the general assembly scheme, the configuration of components and general operation details. Also, the ray tracing simulations and optimizations used in the design of the handheld probe are presented. The data analisys methods, the methods for validation of the system and the clinical study protocol are showed here.

\section{$4.1 \quad$ FLIM setup}

The assembled FLIM system operates on time-domain and uses a laser scanning system for image construction. The decays are measured through direct recording with a fast detector and digitizer. The system general idea and some parts of its setup were based on the one reported by Cheng et al. ${ }^{23}$

A scheme of the FLIM system is presented on Fig. 4.1.

A LabVIEW (National Instruments (NI), USA) program was written to control the hardware and to provide a Graphical User Interface (GUI) (Fig. 4.2) to the microscope operator. The signals are then physically created by a NI Data Acquisition Device (NIDAQ) PXI-6711 (4 analog output channels, 8 digital in/out channels, 12 - bit, $1 \mathrm{MS} / \mathrm{s}$ per Channel). More details about signal generation can be found on Section 4.2. A digital Transistor-Transistor Logic trigger is sent to the Q-switched, frequency tripled, diode pumped Nd:YAG laser (Elforlight SPOT-10-100-355, USA) emitting $<2$ ns pulses at $355 \mathrm{~nm}$. The pulse is emitted approximately $60 \mathrm{~ns}$ after the trigger, but a jitter of a maximum of $1 \mathrm{~ns}$ is expected. To compensate for this jitter, the digitizer (NI PXIe-5160, $1.25 \mathrm{GHz}$ clock, $500 \mathrm{MHz}$ bandwidth, 10 - bit) was not directly triggered by the DAQ signal. Instead of that, the beam is split by a beam sampler (BS) BSF10-UV (Thorlabs, USA) and $\sim 10 \%$ of it is sent to a short rise time photodetector (Detector) (DET10A; Thorlabs, USA), and its signal is used as analog trigger to the digitizer. Both NI-DAQ and digitizer are connected through a fast speed PXI/PXIe to a NI Chassi, which is connected to a x1 PCIe NI adapter in the computer running the system.

Integrated intensity and estimated average lifetime maps are generated in real time and showed at the LabVIEW interface to allow a preview of the acquired data. After 


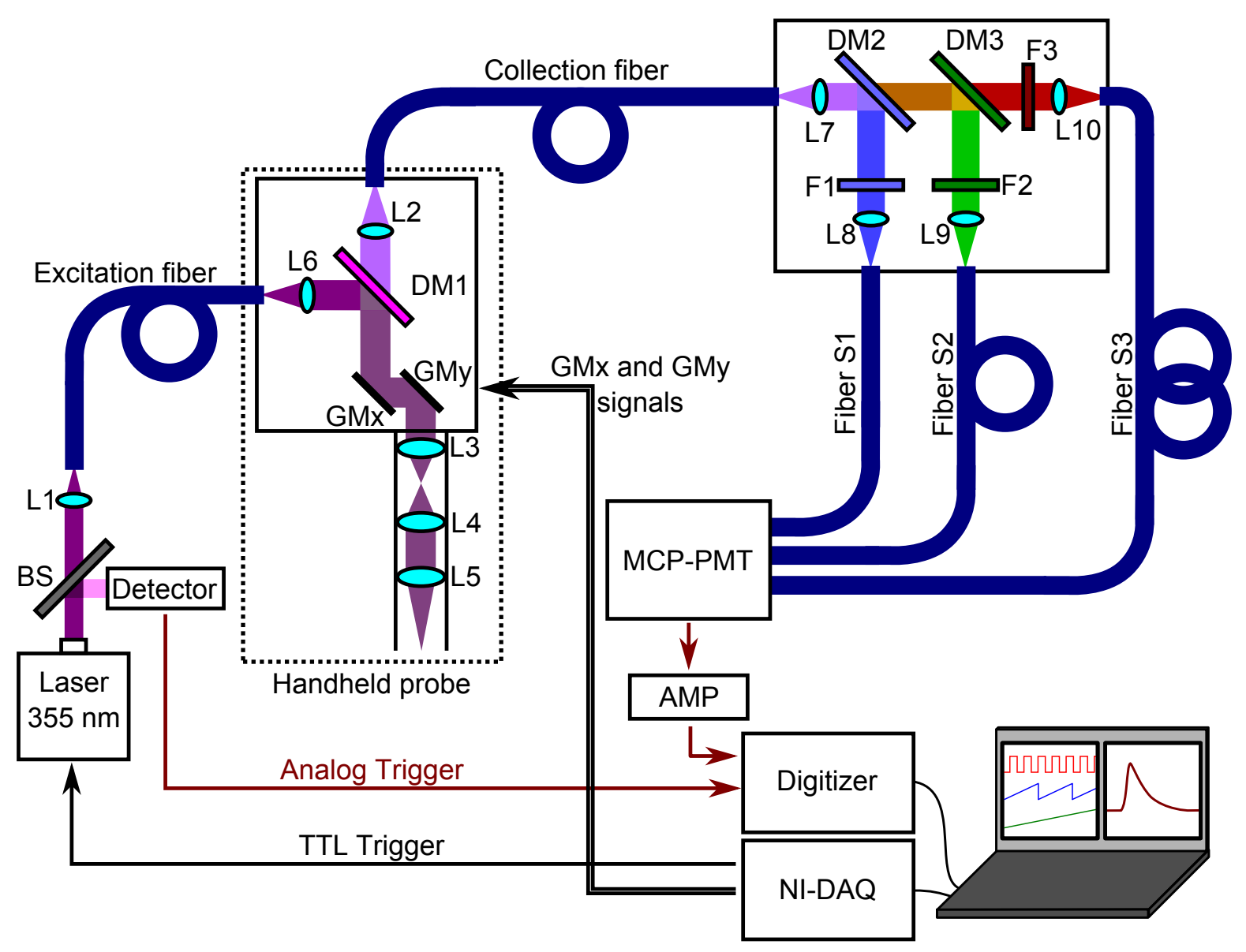

Figure 4.1 - FLIM setup: a computer is used to create the waveforms, that are generated by the signal generation board NI-DAQ, to control the scanning system and to trigger the $\omega$-tripled, Q-switched, $<2$ ns pulse width Nd:YAG laser $(355 \mathrm{~nm})$. A beam sampler (BS) is used to split approximately $10 \%$ of the beam to a short rise time photodetector (Detector), whose signal is used to trigger the Digitizer. The digital signal that triggers the laser is not used directly to trigger the digitizer to compensate for the laser jitter. The laser is coupled into the excitation fiber by the lens L1 and guided to the handheld probe, where it is collimated by L6 and reflected by a dichroic mirror (DM1) to the dual-axis galvanometric mirror system composed of GMx and GMy. Together with the galvo mirrors, the lenses L3 to L5 form a relay scanning system and are also responsible to collect and descan the sample fluorescence, which is transmitted through DM1 and coupled into the collection fiber by the lens L2. The collection fiber guides the sample fluorescence from the handheld probe to a system that splits three different spectral bands using the dichroic mirrors DM2 and DM3, and the filters F1 to F3. The signal is coupled into the fibers S1 to S3 by the lenses L8 to L10. Those fibers have $1 \mathrm{~m}, 13 \mathrm{~m}$ and $25 \mathrm{~m}$ of length respectively. The signal going through each fiber arrives at the MCP-PMT with different delays and can be recorded in sequence by the digitizer after being amplified by a broadband pre-amplifier (AMP).

Source: By the author. 
being measured, the spectral channels are split and processed separately. The integrated intensity $I$ is calculated by the equation

$$
I=\sum_{l=0}^{L-1} y[l],
$$

where $[l]$ is the time-index on the discrete-time basis and $\mathbf{y}$ is the measured fluorescence vector. The estimated average lifetime maps are calculated by the application of the equation 3.21 .

The beam is coupled in a 4 meter long, $50 \mu m$-diameter core excitation fiber (FG050UGA; Thorlabs, USA) by a $20.0 \mathrm{~mm}$ effective focal length (EFL) lens (L1) (LA4647-UV; Thorlabs, USA). This fiber guides the excitation pulse to the handheld probe (more details about the probe design can be found in the Section 4.3. The beam is collimated by an fixed focus fiber collimator (L6) (F220SMA-A; Thorlabs, USA). A dichroic mirror (DM1) (z337rdc; Chroma, USA) reflects the laser light to the galvanometer mirrors system (GMx and GMy) (6200HM40 galvo scanners, 6M2005S20F025A1 mirrors set on a 6102005R20 mount; Cambridge Technology, USA). A set of three lenses (L3 (EFL: $30.0 \mathrm{~mm}$ ), L4 (EFL: $30.0 \mathrm{~mm}$ ) and L5 (EFL: $50.0 \mathrm{~mm})$ ) (LB1258-A, LB1258A and LB1844-A respectively; Thorlabs, USA) work together with the galvos system to act as a relay scanning system. More details about this scanning system are presented on Section 4.3. As the excitation pulse is guided through standard multimode fibers, it arrives unpolarized to the sample.

The fluorescence of the sample is collected by L5, descanned, transmitted through DM1 and coupled into the 4 meters long, $200 \mu m$-diameter core, collection fiber (FG200UEA; Thorlabs, USA) by L2 (F220SMA-A; Thorlabs, USA). The fluorescence signal is guided to the system that splits and filters specific fluorescence bands and couples each one of those bands into different fibers. This is done by the dichroic mirrors DM2 (LM01-427-25; Semrock, USA) and DM3 (FF484-Fdi01; Semrock, USA) and the filters F1 (FF01-390/40; Semrock, USA), F2 (FF01-452/45; Semrock, USA) and F3 (FF01-496; Semrock, USA). The fibers S1 (1 m), S2 (13 m) and S3 (25 m) (FG200UEA; Thorlabs, USA) receive three different spectral bands ((390 \pm 20$) \mathrm{nm},(452 \pm 22) \mathrm{nm}$ and $496 \mathrm{~nm}$ long-pass, respectively).

The excitation wavelength and the spectral windows were chosen targeting three of the most important biological endogenous fluorophores respectively: the collagen, the NADH and the FAD. Collagen is an important structural molecule while the NADH and FAD are related to the cell metabolism. Those three molecules are key fluorophores to track metabolic or structural modifications in living tissues.

The fluorescence signal guided through each fiber arrives at the MCP-PMT (R3809U50; Hamamatsu, Japan) with different delays. In this way, it is possible to record each 


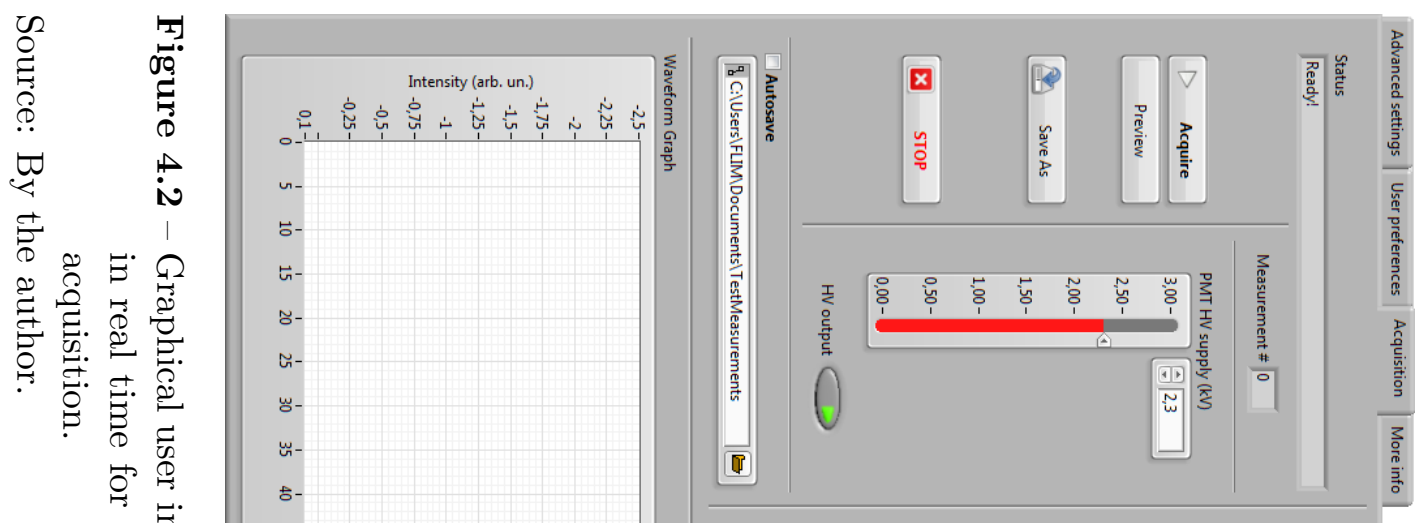

总

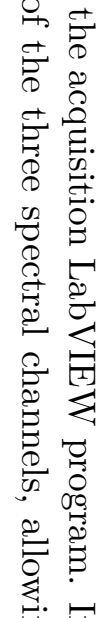

苟

志

융

章

के

늘

过.

\&

吉

ถั

节.

80

产

\&

穴

3 क

응

字

용

के
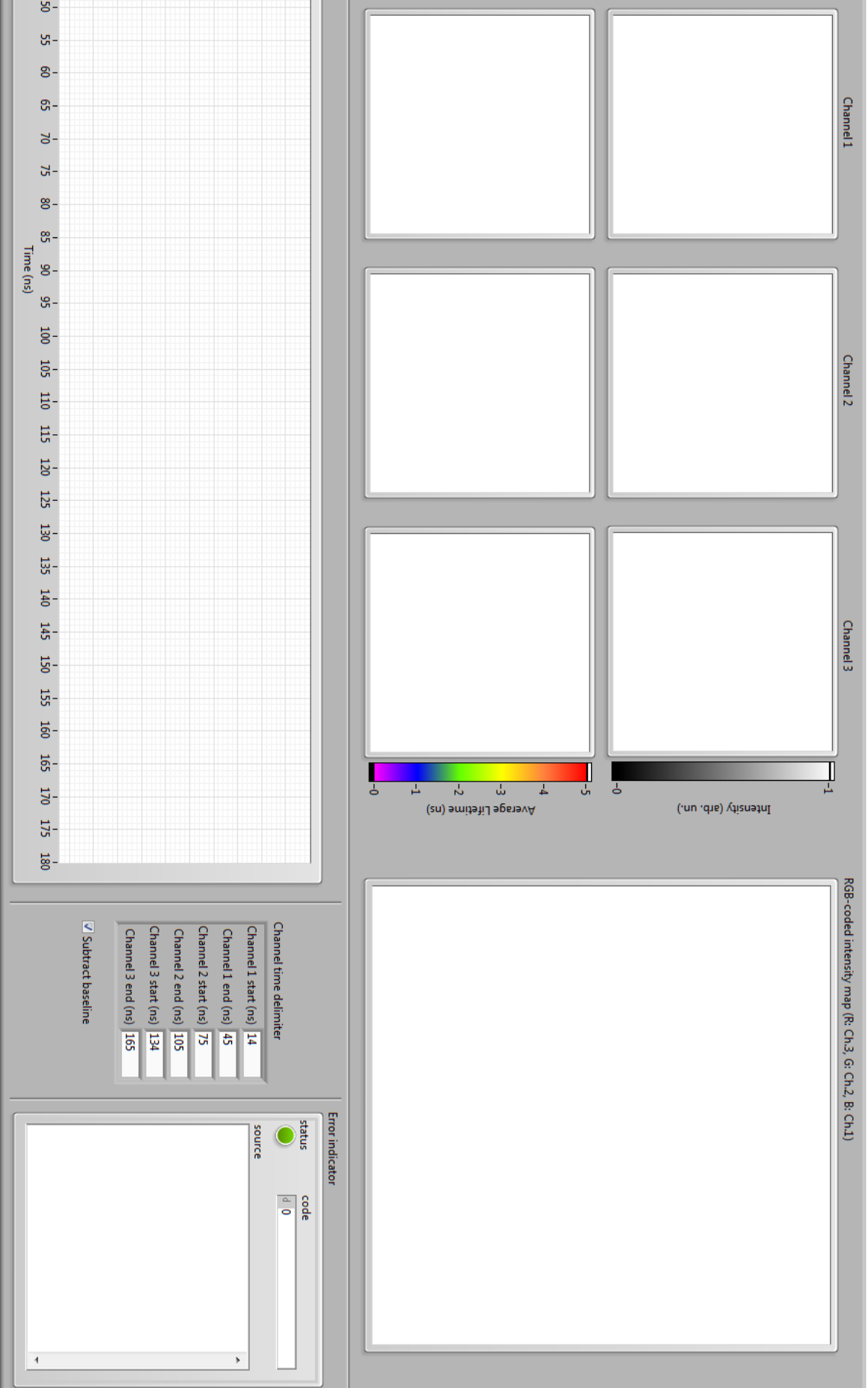
spectral band sequentially, as shown in Fig. 4.3. The signal is amplified by a broadband (50 kHz to $1.5 \mathrm{GHz}) 36 \mathrm{~dB}$ pre-amplifier (C5594-12; Hamamatsu, Japan) and recorded by the digitizer.

The use of delay lines to split temporally the spectral channels acquisition of those channels without the need of three detectors, what would greatly increase the cost of the system and demand acquisition multiplexing, possibly leading to lower acquisition rates.

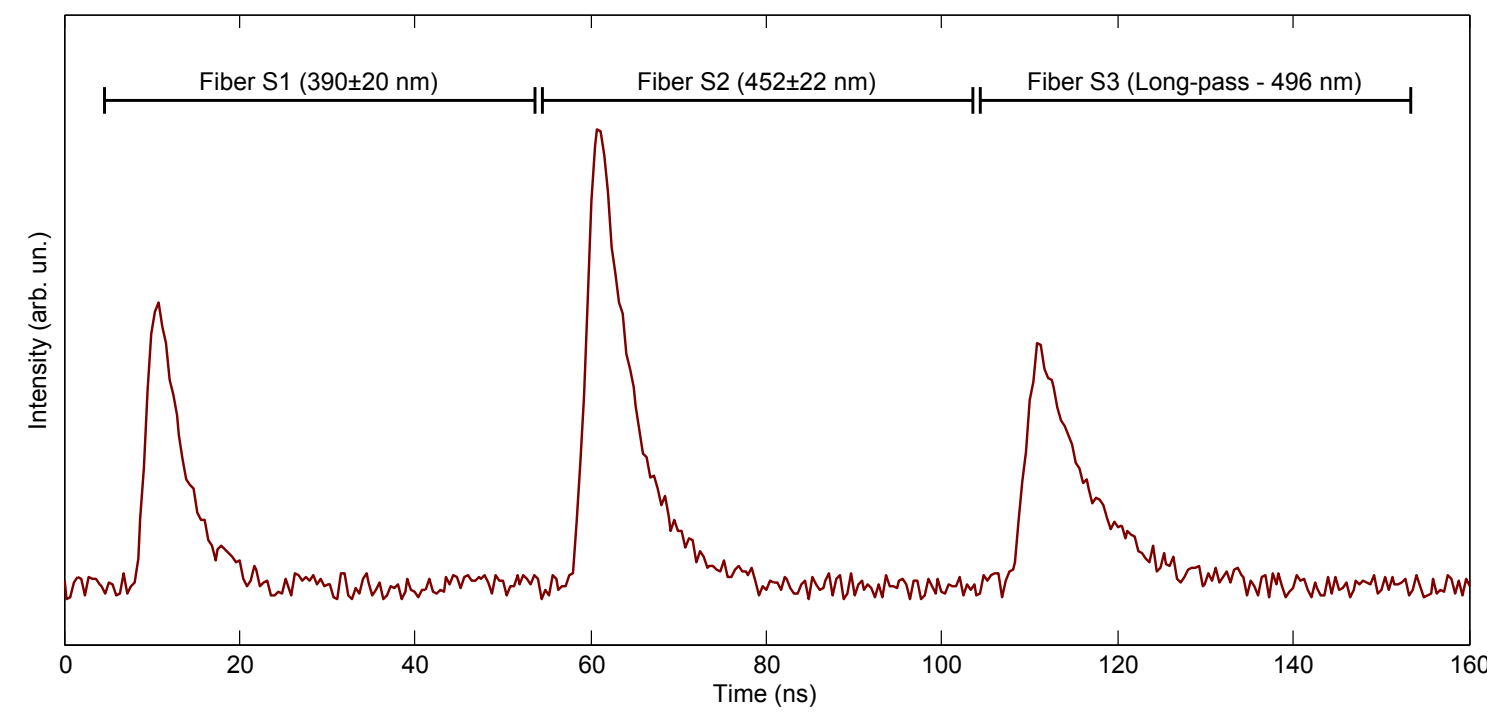

Figure 4.3 - The fluorescence signal is split in three different spectral bands. Each one is sent through fibers with different lengths, producing distinct temporal delays between each one of the signals from each channel arriving at the microchannel plate photomultiplier tube. Using this method, it is possible to acquire decays for each spectral region sequentially as illustrated. (Figure generated using synthetic data.)

Source: By the author.

One of the main differences from the presented system to the one reported by Cheng et $a l .{ }^{23}$ is the digitizer. Cheng used a digitizer with sampling rate of $6.25 \mathrm{GS} / \mathrm{s}$ and bandwidth of $3 \mathrm{GHz}$, while we used one with a much lower sampling rate of $2.5 \mathrm{GS} / \mathrm{s}$ and bandwidth of $500 \mathrm{MHz}$, what greatly reduced the total cost of the system.

Another significant difference is the handheld probe. Instead of using commercial cage systems, we opted to design and fabricate custom optomechanical components to reduce the size of the probe. We also aimed the increase the degrees of freedom of the components to allow a better signal optimization. Additionally, the optical components of our system were chosen to achieve the desired specifications needed for our intended application. More details on this can be found on Section 4.3. 


\subsection{Control and acquisition}

The control of the laser and galvos was performed by multiple channel signal generation through the DAQ. The waveforms were calculated by a routine in LabVIEW using the parameters selected by the user on the GUI. The parameters that can be selected by the user are: digitizer sampling rate $(\mathrm{Hz})$, recording length (ns), DAQ sampling rate $(\mathrm{Hz})$, laser repetition rate $(\mathrm{Hz})$, pixels per line (acquisition mode), lines per frame (acquisition mode), pixels per line (preview mode), lines per frame (preview mode), voltage amplitude for the galvos $\mathrm{X}$ and $\mathrm{Y}(\mathrm{V})$ (each volt corresponds to a mechanical tilt of $1^{\circ}$ ) and flight back time (s).

Three analog channels were used in the control of the two galvos scanning movement and for triggering the laser Q-switch with a digital-like waveform.

The galvanometric mirror responsible for the horizontal scanning (GMx) receives an asymmetric triangle waveform. This signal could be, in principle, a sawtooth waveform, but the sudden flight back creates instability on the galvos driver. The vertical scanning galvo (GMy) receives a linear signal. This signal would ideally be a staircase function, but for the amount of lines per frame we used, the results using a linear function were indistinguishable from when the staircase was used. The reason why the linear function was preferred is that it avoids the current overflow produced on the galvo drivers power supply caused by the abrupt inversion step. The trigger signal was a digital-like function with pulses during the line scan. An illustrative example of the waveforms produced for the acquisition of a $5 \times 5$ pixels image is shown on Fig. 4.4.

The repetition rates were set to $10 \mathrm{kHz}$ and the flight back time to $3 \mathrm{~ms}$ in all tests performed. The power at the sample was set to $10 \mathrm{~mW}$, which allowed us to acquired signal with high quality without offering risks of damage to the samples, and results in $1 \mu J$ per laser pulse. Two acquisition modes are allowed on the GUI: a preview mode and an acquisition mode. The former allows a fast scanning using less pixels (typically $60 \times 60$ pixels, but this value can be adjusted by the user) and it was intended to allow a fast adjustment of the MCP-PMT gain while the latter is for the acquisition of higher quality FLIM images (typically $140 \times 140$ pixels, but also can be changed by the used).

The acquisition was performed by the digitizer operating on pretrigger mode, using a time reference position of $10 \%$ and time interleaved sampling acquisition to obtain sampling rates of $2.5 \mathrm{GS} / \mathrm{s}$ (400 ps). The coupling impedance was set to $50 \Omega$ to match the pre-amplifier requirements.

The acquisition of an image of $60 \times 60$ pixels takes approximately $0.5 s$ while the acquisition of an $140 \times 140$ pixels image requires about $2.4 \mathrm{~s}$, which is similar to the acquisition time of the system presented by Cheng. ${ }^{24}$ However, since our PXI chassi is connected to the PC via an x1 PCIe adapter, the transmission of all the data to it takes 


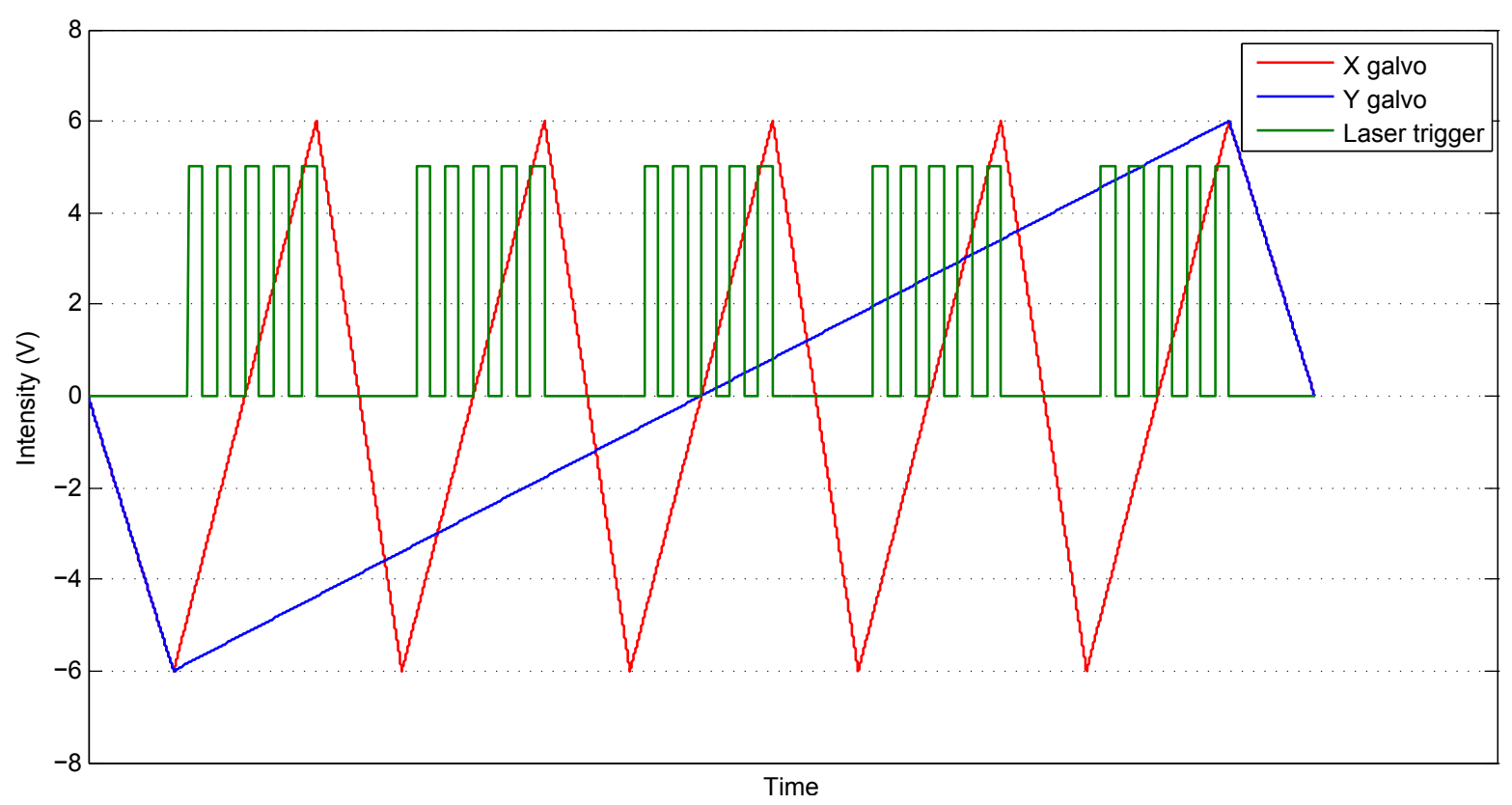

Figure 4.4 - Illustration of signals generated to control both galvos and to trigger the Qswitched laser for the acquisition of a $5 \times 5$ pixels image in arbitrary time units. An asymmetric triangle waveform is used to control the $\mathrm{X}$ scanning while a linear function is used for the $\mathrm{Y}$ scanning. Digital pulses were used to trigger Source: By the author. the laser during the scan but not during the flight back.

roughly three times the scanning time, what represents the bottleneck of our system in terms of image acquisition rate. This could be improved by using PXI controllers, which are not limited by the x1 PCIe data transfer rate.

\subsection{Handheld probe design and optimization}

The main goal of our system is to be applied in a clinical setting. In this case, the handheld probe requires a long $(>10 \mathrm{~cm})$ rigid endoscope to allow flexible use of the system, including the imaging of internal areas like the cervix. So a scanning system composed of two relay lenses (L3 and L4) and one objective lens (L5) was proposed. The general idea of the handheld probe was shown in Fig. 4.1. The general design of the handheld probe is also tightly related to the one presented by Cheng et al. ${ }^{23}$ but the optomechanical components used were custom designed and fabricated for optimized probe size.

To choose the set of lenses and angular scanning ranges to acquire the desired field of view (FOV) and resolution, that in our case is around $8 \mathrm{~mm}$ and $100 \mu \mathrm{m}$ respectively, the software Zemax (Zemax, LLC, USA) was used. To simulate the fiber output, a circular light source with diameter of $50 \mu \mathrm{m}$ and isotropic emission half-angle angle of $12.7^{\circ}(0.22 \mathrm{NA})$ was used. All the spheric (L3-L5) and aspheric lenses (L2) were 
perfectly reproduced with information available on the vendors website. The position and angles of the galvos were also carefully reproduced. The result of the ray tracing simulation can be seen on Fig. 4.5, where each ray color represents the result for multiple configurations of the galvos.

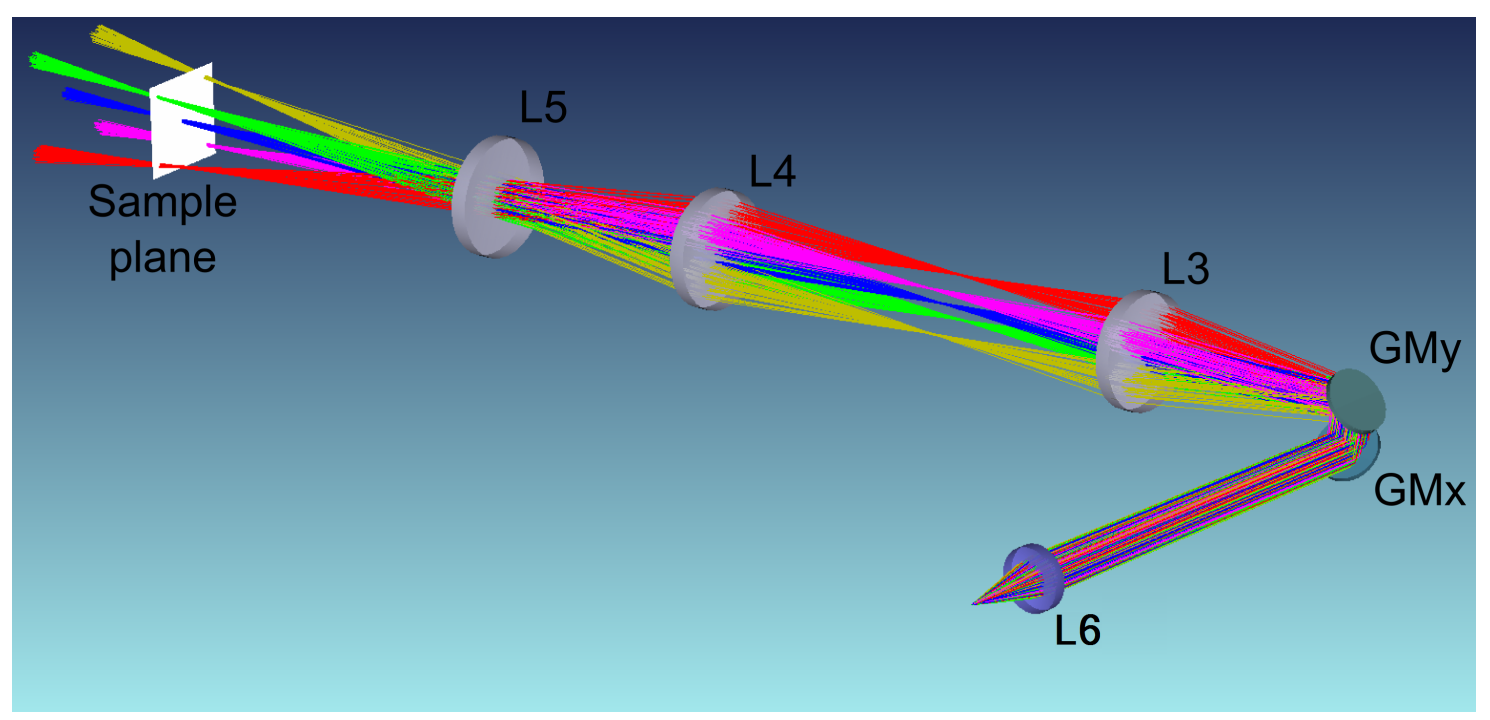

Figure 4.5 - Zemax ray tracing of the handheld probe. The fiber output was modeled as a circular light source with diameter of $50 \mu \mathrm{m}$ and emission half-angle of $12.7^{\circ}$ $(0.22 N A)$. Each ray color represents the result for one configuration of the Source: By the author. galvos GMx and GMy.

The lens L3, with effective focal length $f_{3}$, is placed at a distance $f_{3}$ of the point between mirrors (since the galvos have different distances to the lens L3, there is not an absolute conjugated point as if it was an afocal beam relay system, ${ }^{55}$ but this is a valid approximation as the next results will confirm). The lens $\mathrm{L} 4\left(\mathrm{EFL}=f_{4}\right)$ is placed to act as a telescope together with L3. The objective lens L5 $\left(\mathrm{EFL}=f_{5}\right)$ is placed at the focal point of L4 so that the beam always crosses its center as illustrated on Fig. 4.6.

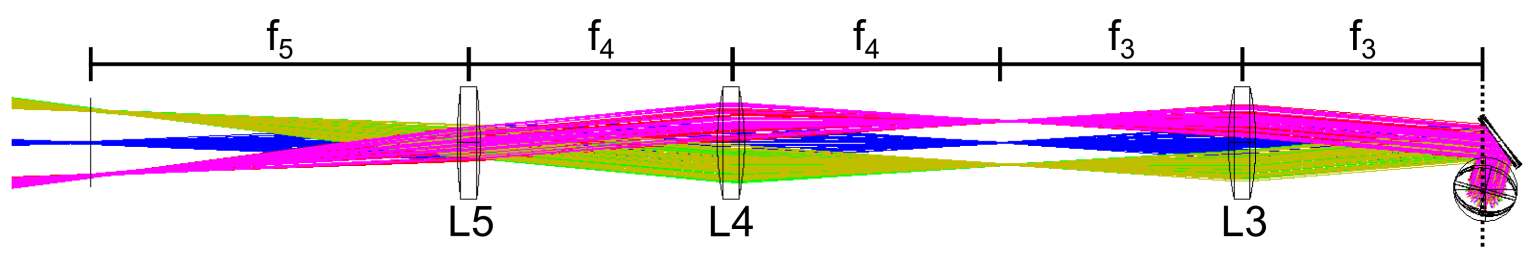

Figure 4.6 - The lenses L3 and L4, with effective focal lengths of $f_{3}$ and $f_{4}$ respectively, act as a telescope conjugated with the galvos system. The lens L5 (effective focal Source: By the author. length of $f_{5}$ ), used as an objective lens, is placed on the focal point of L4.

Since the lenses L3-L5 are simple bi-convex spherical lenses some spherical aberration is expected. The effects of this aberration can be three:

- the loss of orthogonality of X and Y scanning: the field curvature would cause the spot to move along an arc during the line scan; 
- the loss of linear dependence of the galvos angles with spot position (also related to the previous item);

- the dependence of the spot size on the position of the spot,

being all these effects obviously more noticeable on the periphery of the image.

The orthogonality of the GMx and GMy scanning was also evaluated on Zemax with the simulation of two-dimensional maps where the independent variables were the mechanical tilt of both galvos and the dependent variables were the horizontal and vertical position of the spot center on the sample plane. The results are presented in Figure 4.7 and show that the $\mathrm{X}$ position of the spot is highly independent of the tilt on the GMy galvo and vice versa.
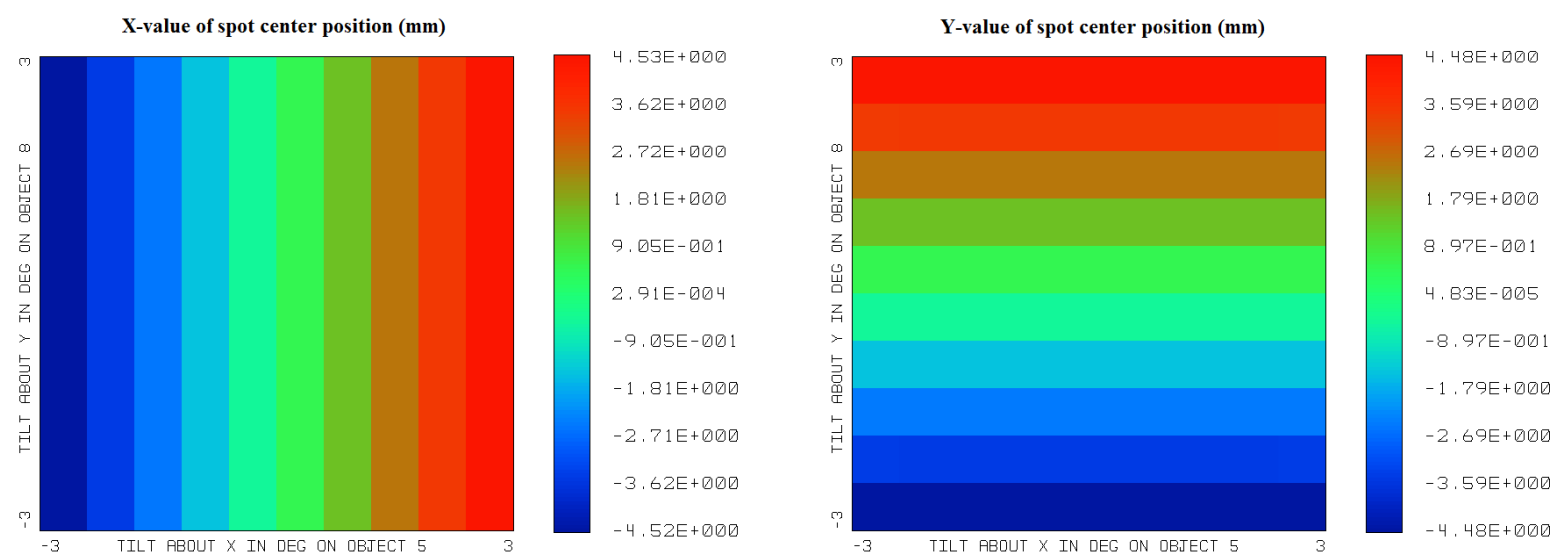

Figure 4.7 - The X position (left) and Y position (right) only depend on the tilt on the GMx and GMy galvos respectively.

Source: By the author.

The linearity of the spot position with the scanning angle was also evaluated with a Zemax simulation. The results, shown in Fig. 4.8, indicates that the system is highly linear, allowing us to create a linear equivalence between angular scan range and FOV.

The dependence of the spot size (which is also the resolution of the microscope) with the spot position was also analyzed. To perform that, the root mean square width of the spot was simulated on Zemax.

A mean resolution of around $\sim 120 \mu \mathrm{m}$ was achieved, but in this simulation the effects of spherical aberration (together with comatic aberration) becomes clear. In the corners of the image, the RMS spot size reaches values of approximately $250 \mu \mathrm{m}$ (Fig. 4.9).

With all the parameters for achieving the desired FOV and resolution known, the handheld probe was designed and machined in aluminum. All the optomechanical components were also custom-designed because the standard commercial solutions would 

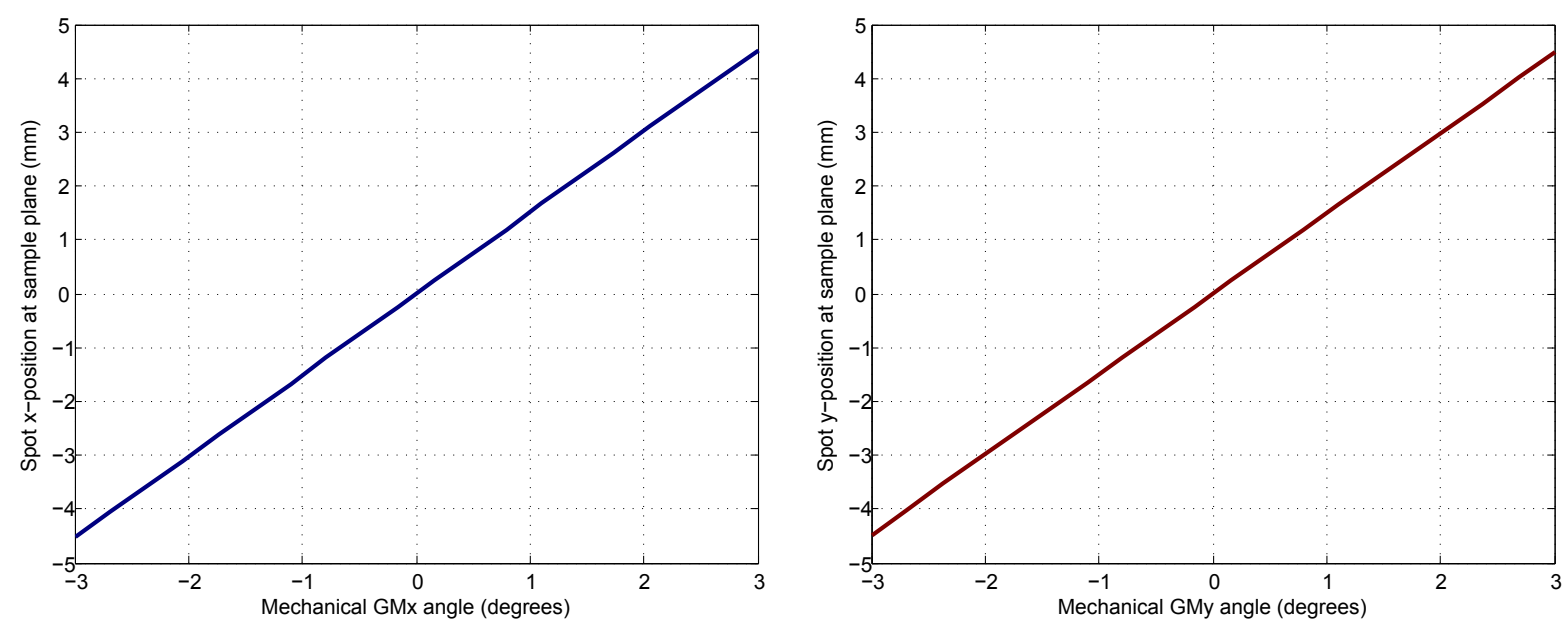

Figure 4.8 - The X position (left) and Y position (right) of the spot center varies linearly with the mechanical tilt of the GMx and GMy galvo, respectively.

Source: By the author.

require much more space, increasing the size and weight of the probe. A photograph of the probe is shown in Fig. 4.10 (details of the components are discussed on Fig. 4.1).

The total weight of the probe without fibers and cables is $280 \mathrm{~g}$. An autoclavable stainless steel tip is used to protect the endoscope and avoid contamination between patients, which is absolutely necessary for clinical application.

The handheld probe is connected to the other parts of the system by the galvo scanners cables and by the excitation and collection optical fibers. All those cables and fibers are $4 \mathrm{~m}$-long to allow a significant working freedom, what is essential when imaging certain regions of patients with reduced mobility.

\section{$4.4 \quad$ FLIM data analysis}

The processing of the FLIM datasets was done using algorithms implemented in MATLAB (The Mathworks, USA). The direct implementation of channel-by-channel, pixel-by-pixel analysis using MATLAB built-in fitting functions returned very precise results at the cost of long computing times. Using this method, the fitting of each pixel (for each channel) took about $100 \mathrm{~ms}$, so the analysis of a single $140 \times 140$ pixels image took more than 1.5 hour.

To reduce the computation time necessary to analyze each image, a custom implementation of the Gauss-Newton algorithm (discussed on the subsection 3.4.2) was written. In our algorithm, each iteration computes every parameter for the whole image at once, instead of optimizing the parameters of each pixel at a time. By using this method, the computation time for the analysis of each $140 \times 140$ pixels image dropped down to less than 2 minutes.

Exponentials of different orders $(N)$ were tested on the analysis of the acquired 


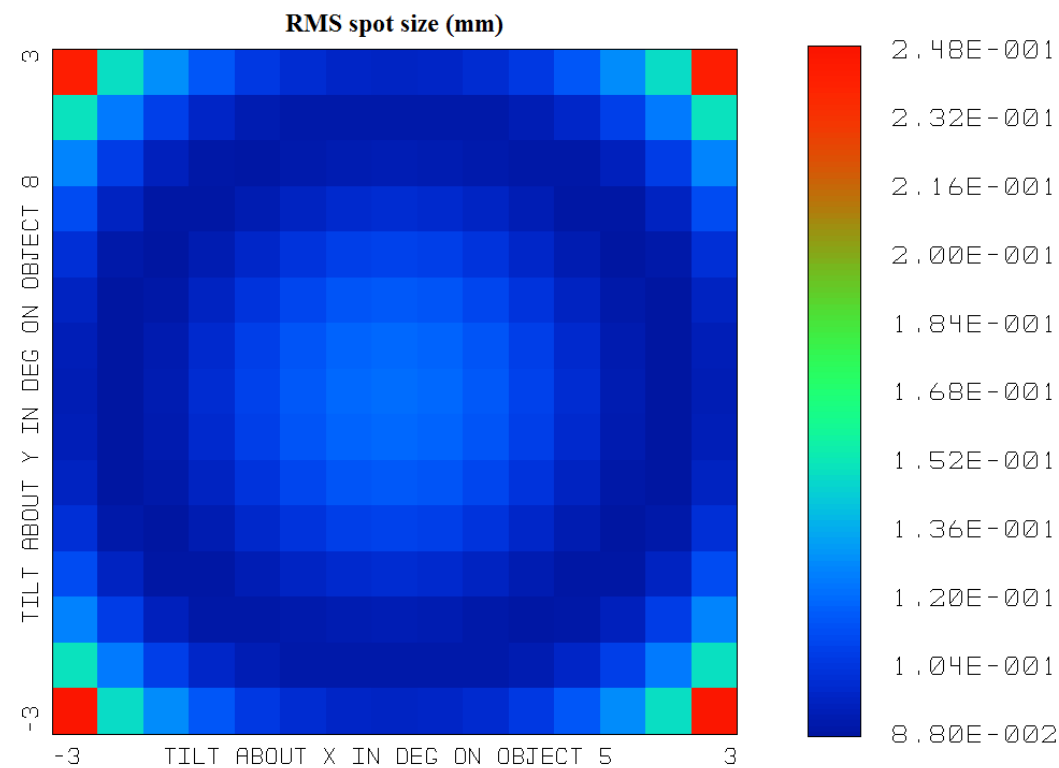

Figure 4.9 - RMS size of the spot on the sample plane. The mean resolution lies around $\sim 120 \mu \mathrm{m}$, but due to optical aberrations it reaches approximately $250 \mu \mathrm{m}$ on the corners of the image.

Source: By the author.

data. Based on the convergence stability of the fitting and on the fitting correlation, we choose a biexponential model $(N=2)$ to fit our data. The results will be displayed as a combination of integrated intensity maps (calculated with the equation 4.1), lifetimes $\tau_{n}$, and normalized weights $a_{n}$, where:

$$
a_{n}=\frac{c_{n}}{\sum_{m=1}^{N} c_{m}},
$$

where $\mathbf{c}$ is the vector of weighting coefficients.

We also performed some tests with the lifetime estimation method based on the phasor approach (Equation 3.24).

\subsection{On-the-bench setup}

A simplified on-the-bench setup was assembled for preliminary tests. This was done to test and optimize the LabVIEW program parameters and the electronics setup in a more controllable setting.

A single lens with EFL of $50 \mathrm{~mm}$ was used in front of the galvos to perform a simple $f \cdot \tan \theta$ scan. The same dichroic mirror (DM1) was used to reflect the excitation and transmit the fluorescence. The fluorescence was filtered by a long-pass filter with edge at $496 \mathrm{~nm}$ and coupled to the MCP-PMT without any fibers. In this way, the initial 


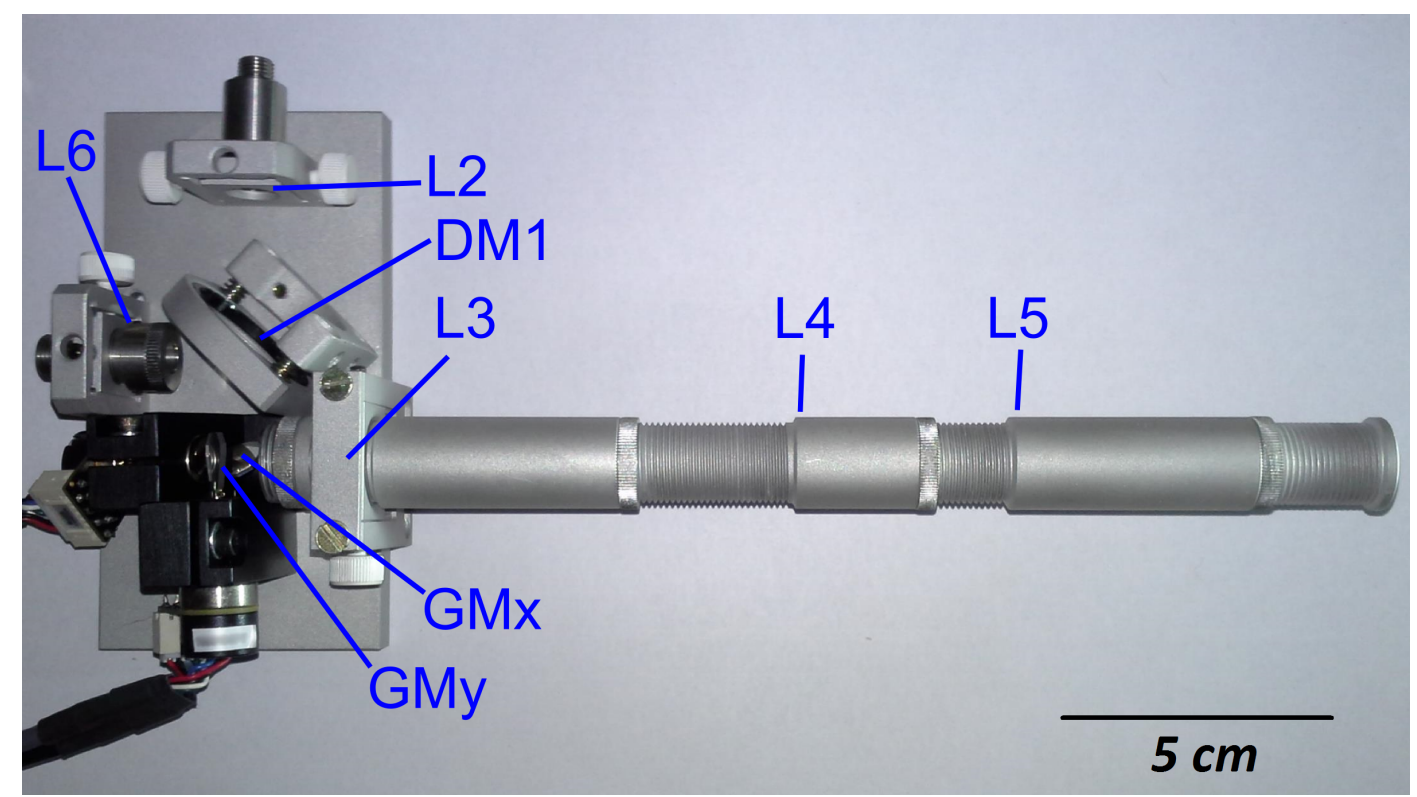

Figure 4.10 - FLIM handheld probe - The probe and its optomechanical components were custom designed and built entirely in aluminum to result in low size and weight (280 $\mathrm{g}$ without fibers and cables). The lens L6 collimates the excitation beam, which is reflected by the dichroic beam splitter DM1. The galvos GMx and GMy perform the scanning and the lenses L3 and L4 act as a scanning relay for the objective lens L5. The signal is descanned and collected by the lens L2.

Source: By the author.

tests were done using a single spectral channel.

The system was then assembled on a custom designed mobile platform. Details of the platform in which the system was assembled on are discussed in the next section.

\subsection{Mobile platform for the FLIM system}

The on-the-bench FLIM setup had several limitations in terms of the experiments that could be performed with it. One of the main limitations of this setup was that of commercial microscopy systems: the sample had to be positioned on the microscope, and not the other way around. Any experimenting would have to be done on top of an optical table, what impose a significant set of limitations to the experiments. Additionally, optics laboratories often does not meet the requirements to allow clinical procedures to be performed.

To address those issues, a custom designed and custom built platform was designed to house the FLIM system. Not only this platform allows the whole FLIM system to be easily transported to other rooms, but it also allowed the FLIM system to operate entirely independently from external resources, except for an $127 \mathrm{~V}-60 \mathrm{~Hz}$ power outlet. A room temperature no higher than $30{ }^{\circ} \mathrm{C}$ is preferred for the system operation. The frontal view of the mobile platform is shown on Figure 4.12. 


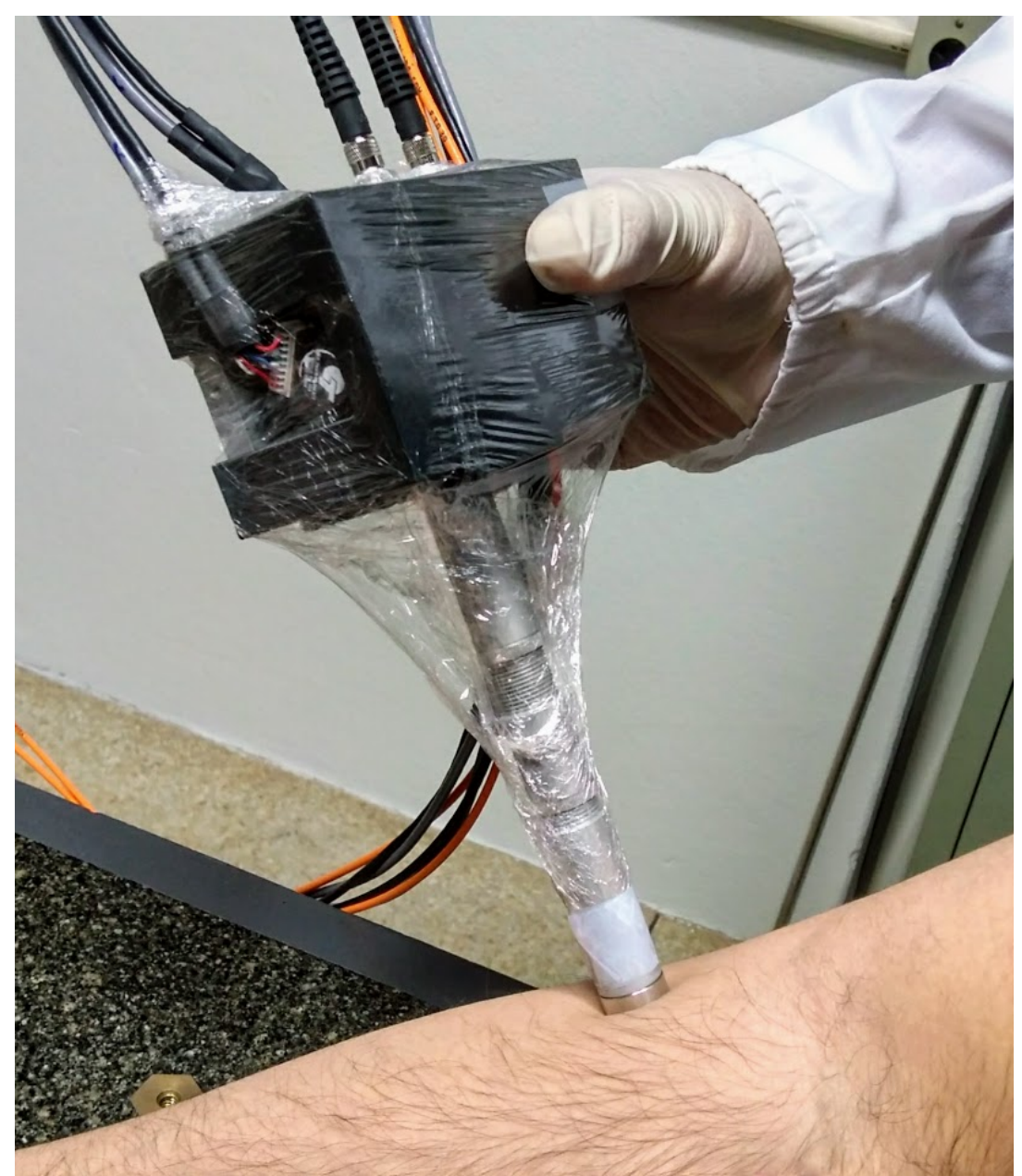

Figure 4.11 - The FLIM system handheld probe was designed to allow in vivo imaging. It is connected to the remaining parts of the system by $4 \mathrm{~m}$-long cables and optical fibers patch cables, making it possible to be used to image skin lesions in a clinical setting.

Source: By the author.

This design presents the usage flexibility of an already reported system featuring a handheld probe ${ }^{23}$ without any restrictions of the system to an optical table or any other piece of lab equipment. This creates the possibility of employing the system in different settings.

The cart has three separate enclosed compartments. The computer and laser power supply are located on the lowest compartment. NI Chassi, high voltage power supply for the MCP-PMT, MCP-PMT, galvos drivers, and power supply for galvos drivers are located on the middle level. On the top level, all the optical components are mounted on a aluminum optical breadboard. The computer screen, keyboard and mouse are positioned on top of the cart to allow easy use. The components on the breadboard are shown in Figure 4.13. 


\section{$4.7 \quad$ In vivo imaging}

The system, already operating with the handheld probe, was finally tested with in vivo skin imaging on healthy volunteers to evaluate signal quality, SNR and contrast in benign lesions.

We also imaged skin tumors induced in mice to evaluate the system sensibility to modifications caused by early stage tumors. The animals used in these experiments were BALB/c nude mice. These animals lack or have a deteriorated thymus, thus being immunodeficient and allowing studies with heterotransplanted tumors (tumors from other species transplanted to the animal). For this study, human Squamous Cell Carcimoma (SCC) (A-431, ATCC ${ }^{\circledR}$ CRL-1555 ${ }^{\mathrm{TM}}$ ) and pigmented melanoma (B16F10, ATCC ${ }^{\circledR}$ ) tumors were induced by intradermal injection of one million cells in $30 \mu L$ of phosphatebuffered saline solution. For the tumor induction, the animals were anesthetized with isoflurane at $5 \%$ for induction and $2 \%$ for maintenance. The same anesthetizing protocol was used for the imaging of the tumors. All animal experiments followed ethical procedures and were approved by Comissão de Ética no Uso de Animais do IFSC (Sao Carlos, Brazil) (Annexes A and B).

The FLIM system was moved to the Department of Skin and Soft Tissues, at Hospital Amaral Carvalho (Jahu, SP, Brazil), that has as main focus the diagnosis and treatment of multiple types of cancer. This preliminary study was carried out in collaboration with the dermatologist Dr. Ana Gabriela Salvio. Our goal is to perform a study in the future evaluating the potential of the technique in differentiating clinically similar skin lesions. For now, some pilot measurements were performed to evaluate the system clinical performance for specific lesions such as nodular BCC (Basal Cell Carcinoma), sclerodermiform BCC, superficial BCC, seborrheic keratosis, Bowen's disease, dysplastic nevus, and melanoma.

The lesions to be imaged were selected by Dr. Salvio based on their clinical relevance and incidence rate. The technique, its associated risks, and the goals of this study were explained for the patients to decide whether or not to join as a volunteer. Every question of the patients regarding the safety of the measurement, the protocol, or somehow related to the study were promptly answered by the researchers involved.

None of the FLIM measurements taken during this pilot clinical study were used in any way by the medical or nursing team, nor it influenced the treatment or the clinical protocol regarding the procedures the patients underwent.

The diagnosis of each lesion was given by Dr. Salvio based on the clinical inspection followed by dermatoscopy. The area of the lesion was demarcated by the dermatologist for the FLIM imaging. In the case of small lesions (smaller than the FOV), we positioned the probe centered on the lesion. On larger lesions, we measured the borders of the 
lesion so each image contains both healthy tissue areas and lesion areas. Some lesions were cleaned with gauze and saline solution (mainly to remove tissue crusts) before the imaging. 


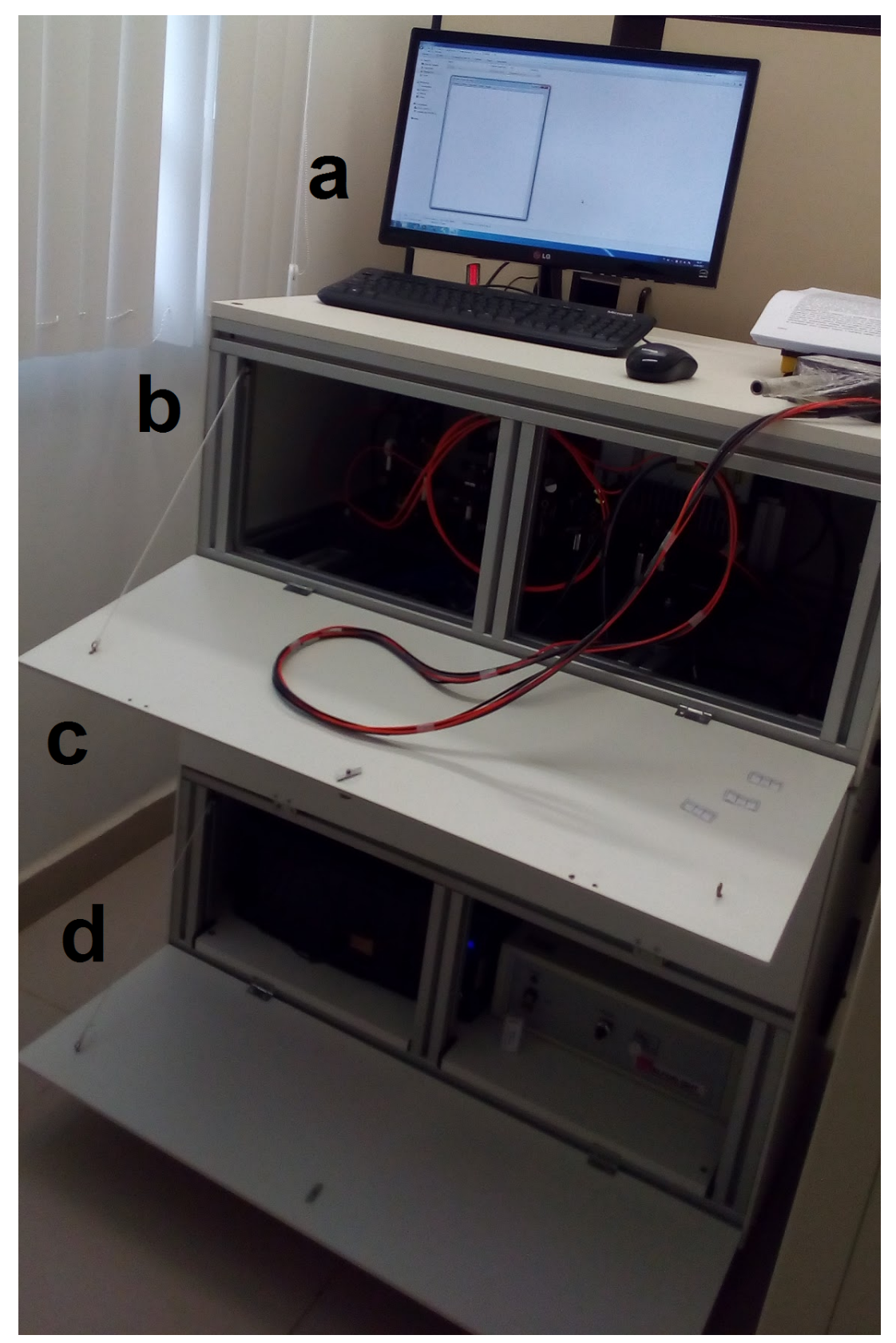

Figure 4.12 - The FLIM system was assembled onto a mobile platform that allows the system to be moved to and operated on typical clinical settings. The only external requirements is an $127 \mathrm{~V}-60 \mathrm{~Hz}$ power outlet and temperatures preferably no higher than $\sim 30{ }^{\circ} \mathrm{C}$. The mobile platform features three separate enclosed compartments. The computer and laser power supply are located on the lowest compartment (d). NI Chassi, high voltage power supply for the MCPPMT, MCP-PMT, galvos drivers, and power supply for galvos drivers are located on the middle level (c). On the top enclosed level (b), all the optical components are mounted on a aluminum optical breadboard. The computer screen, keyboard and mouse are positioned on top of the cart to allow easy use (a).

Source: By the author. 


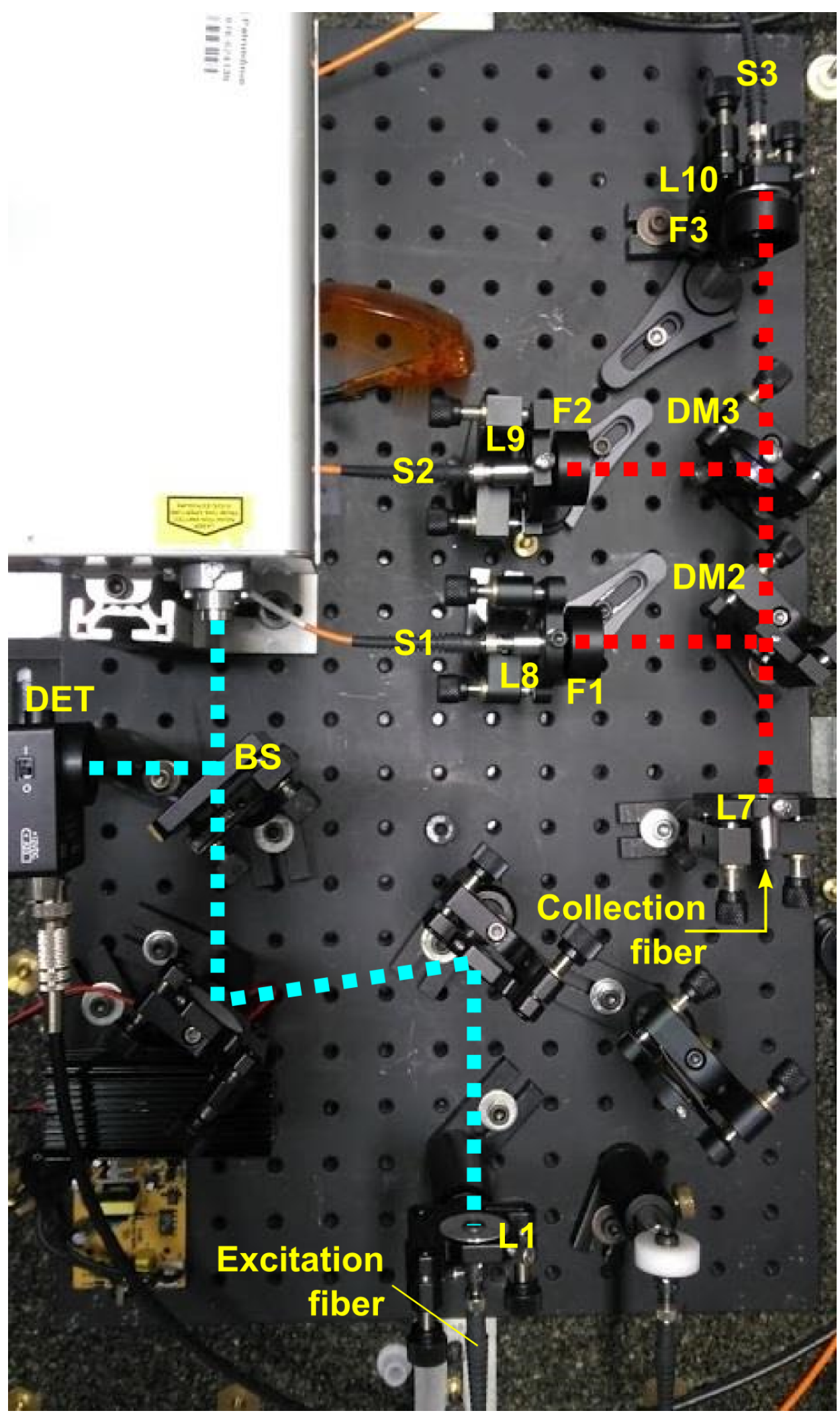

Figure 4.13 - Optical components on the optical breadboard of the FLIM system mounted on an optical breadboard. For a description of the components, please refer to Figure 4.1 on page 44 .

Source: By the author. 


\section{$5 \quad$ Results and discussions}

The characterization of both the on-the-bench setup and the final setup, featuring the handheld probe, will be presented in this chapter. We will also present some validation measurements at an animal model and the results of a pilot clinical study.

\section{$5.1 \quad$ On-the-bench setup}

Some preliminary tests and characterization measurements will be presented in this section.

\subsubsection{IRF characterization}

The most straightforward method to determine the IRF is the direct measurement of a laser pulse. This procedure takes into account every contribution to the system IRF as laser pulse width, finite amplification and digitizing bandwidth and detection response time, but also requires the assumption that the MCP-PMT IRF does not depend on the wavelength, which is true (to a measurable extent) in our case.

To perform the IRF characterization, we placed a mirror in front of the scanning lens and the galvos were kept centered. Since the MCP-PMT sensitivity is incompatible with a direct measurement of the laser light, the beam intensity was attenuated to a level in which our neutral density filters could not reach. So, instead of using NDFs, the same dichroic mirrors and filters were kept on place so that only a few photons of the laser statistically manage to go back through the DM1 and across the long-pass filter at $496 \mathrm{~nm}$ in front of the MCP-PMT. This procedure allowed to perform the acquisition of a good quality signal.

The on-the-bench system measured IRF has a full width at half maximum (FWHM) of $1.85 \mathrm{~ns}$, as shown in Fig. 5.1. This signal was used on the fitting of the FLIM data sets.

This IRF curve is valid solely for the presented on-the-bench setup. Since the fibers will cause dispersion on the excitation and, mainly, on the fluorescence signal, the IRF will have to take it into account. So, the IRF of the FLIM final setup will be measured for each spectral channel since each one is collected by fibers with significantly different 


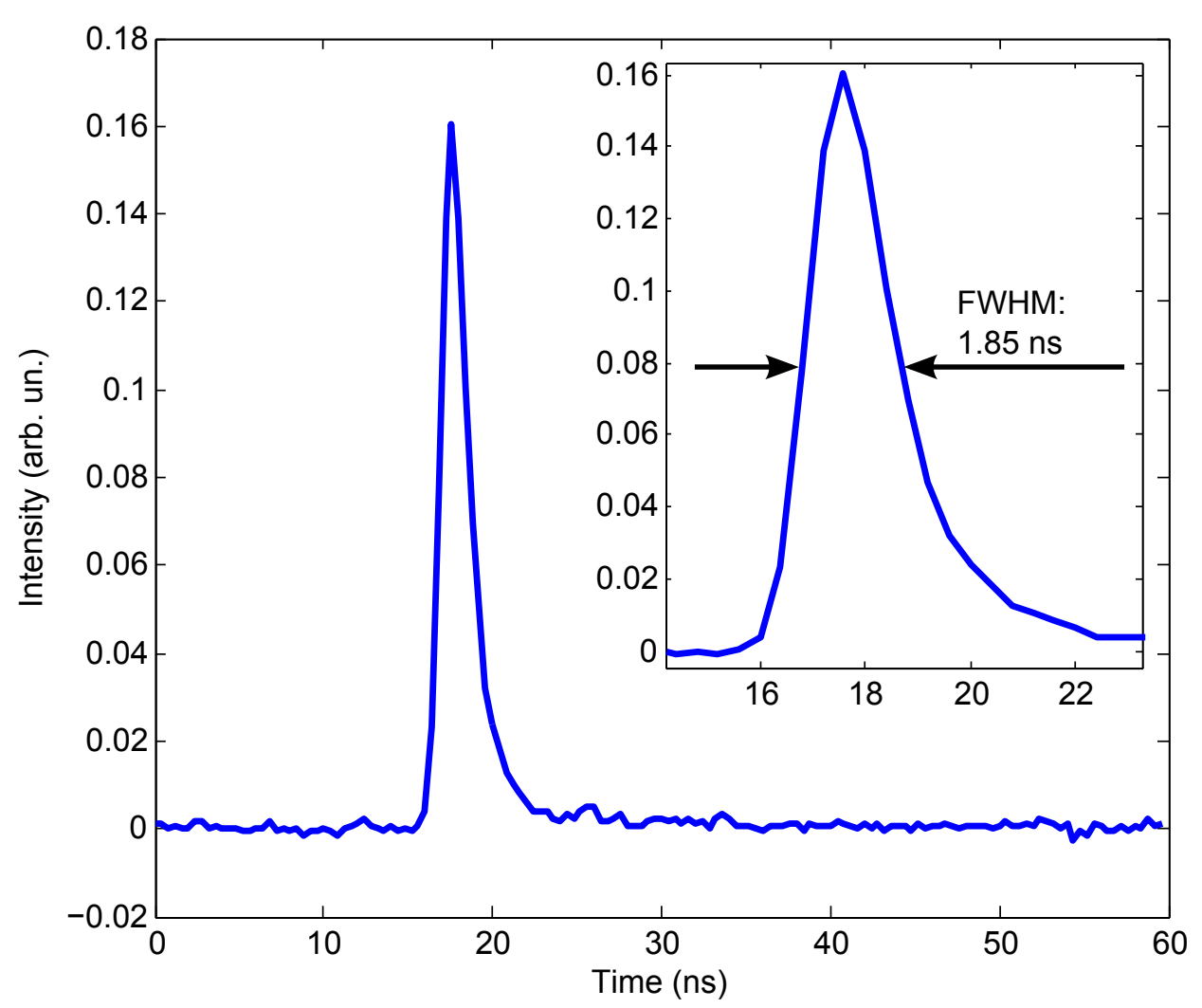

Figure 5.1 - The FWHM of the IRF measured through the direct recording of the laser pulse is $1.85 \mathrm{~ns}$.

Source: By the author.

lengths.

\subsubsection{Imaging}

A validation of the assembled system temporal resolution was performed with the measurement of the fluorescence lifetime of a solution of Rhodamine 6G in ethanol with undetermined concentration. This measurement was performed with both the present system and with a commercial TCSPC spectroscopy system (Becker \& Hickl, Germany) using excitation at $378 \mathrm{~nm}$ (this system is further described by Nogueira et $a l .{ }^{32}$ ). The analysis of the curves acquired with the presented system and with the commercial TCSPC system revealed a fluorescence lifetime of $6.0 \pm 0.1$ and $5.9 \pm 0.1$ ns respectively.

The signal-to-noise ratio of the system was also estimated to be $49 d B$.

After setting the parameters for the adequate acquisition of fluorescence decay curves, the scanning system was tested by performing a full FLIM acquisition. Each signal was integrated over time and an integrated intensity image was produced.

A Thorlabs alignment card was scanned and the first FLIM image acquired with this system was successfully obtained. A picture of the card placed in front of the scanning lens and the integrated intensity image are shown in Fig. 5.2. 

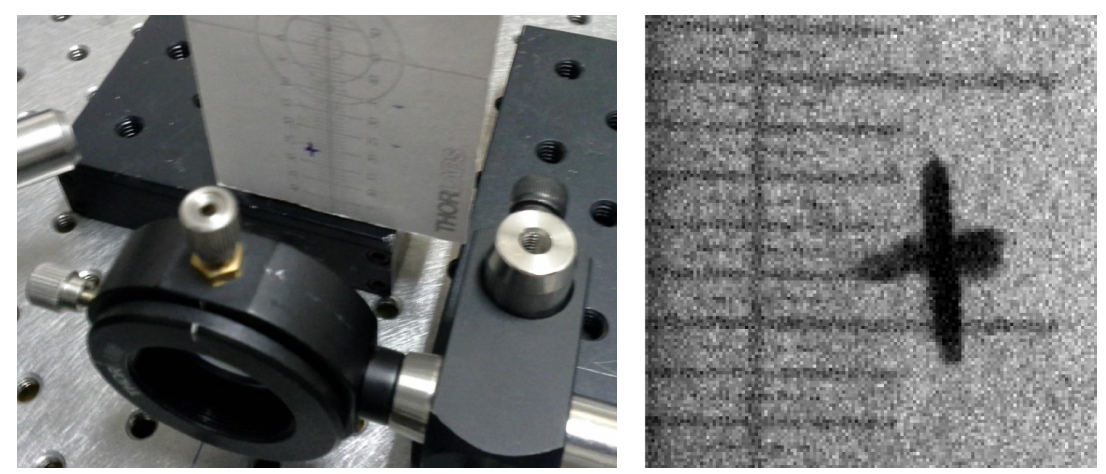

Figure 5.2 - Alignment card placed at sample position in front of scanning lens (left) and integrated intensity image of the first FLIM data set acquired with the system (right).

Source: By the author.

To demonstrate contrast with both intensity and lifetimes, a test sample with inkjet printed characters and marked with yellow highlighter pen was used. The integrated total intensity map is shown on Fig. 5.3.

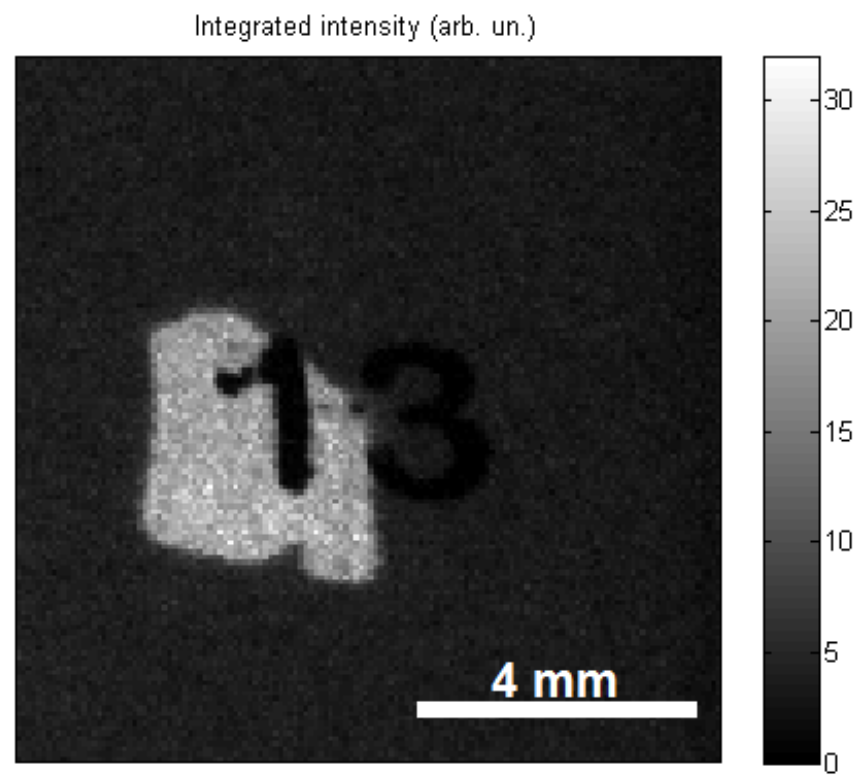

Figure 5.3 - Integrated intensity map of test sample. The region with higher fluorescence intensity is marked with yellow highlighter pen and the visible characters are inkjet printed on the paper.

Source: By the author.

The fluorescence lifetime of paper is much shorter than that from the highlighter ink. The normalized fluorescence decay curves of two points (one with with and one without highlighter ink) are shown in Fig. 5.4. The average lifetimes calculated for those points are 0.7 ns and 2.7 ns respectively.

By evaluating the average lifetime in each pixel using the equation 3.21 of the image, it is possible to build an average lifetime map as shown in Figure 5.5. 


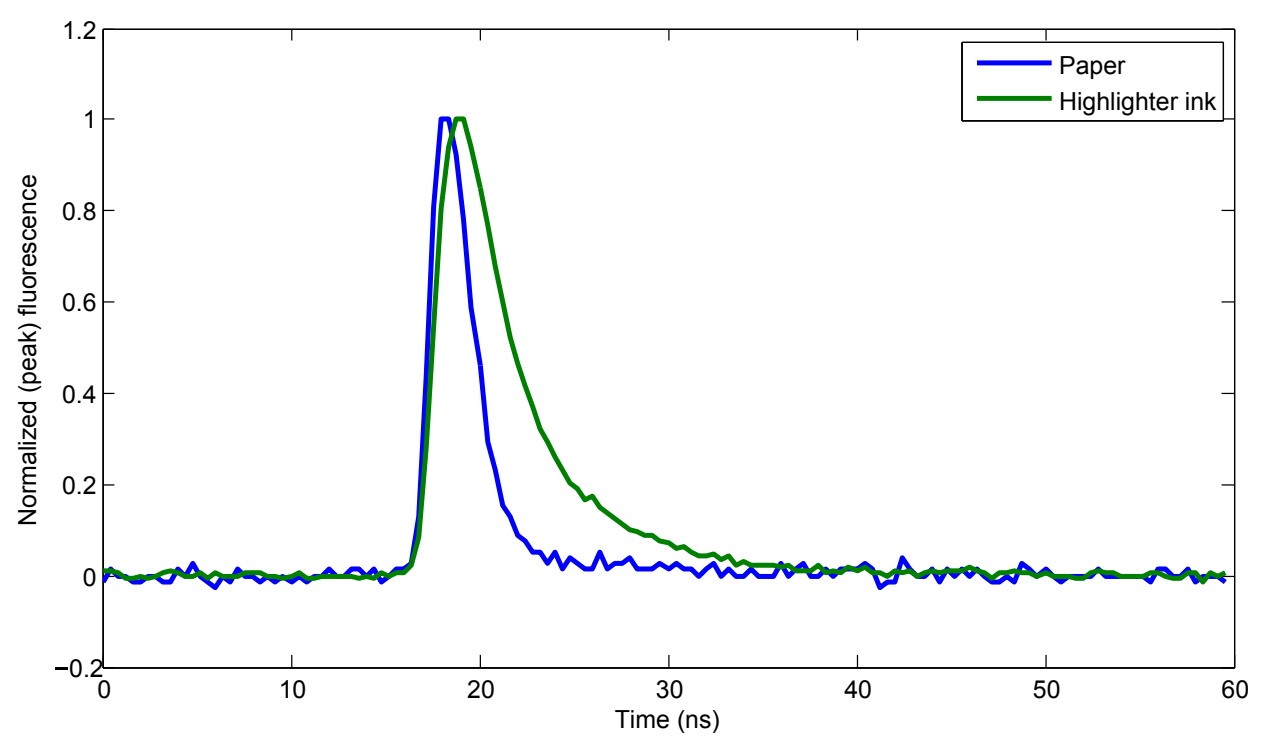

Figure 5.4 - The fluorescence decay of the highlighter ink is much slower than the one of paper. The average fluorescence lifetime calculated from those curves are $0.7 \mathrm{~ns}$ for the paper and $2.7 \mathrm{~ns}$ for the highlighter ink.

Source: By the author.

However, some pixels present low fluorescence intensity and the low signal-to-noise ratio compromises the precision of the lifetimes calculation. This causes some artifacts that appear on the lifetime map, usually observed as points with meaninglessly long lifetimes (sometimes of tens of thousands of nanoseconds).

\subsection{Mobile platform multispectral FLIM system}

After the initial tests with the simplified on-the-bench setup, we designed and built a mobile platform to allow in vivo measurements. This setup has three spectral channels and is described on the Sections 4.1 and 4.6. A scheme is presented on Figure 4.1. The characterization and some in vivo imaging tests are presented on this section.

\subsubsection{Channels spectral efficiency}

The spectral efficiency of each channel was measured (Figure 5.6). To do that, a broadband light source was coupled into the the collection fiber, while the other end of the fiber was connected to a spectrometer (USB2000, Ocean Optics, USA). The spectrum of the coupled broadband light was acquired. Next, the collection fiber was disconnected from the spectrometer and connected to the entrance of the multispectral module. The fibers of the three channels were connected (one at a time) to the spectrometer, so the spectrum of the light coming out of the fibers of each channel was measured. Finally, we calculated the spectral efficiency by normalizing the spectrum of the light coming out of each fiber by the spectrum of the coupled broadband light. 


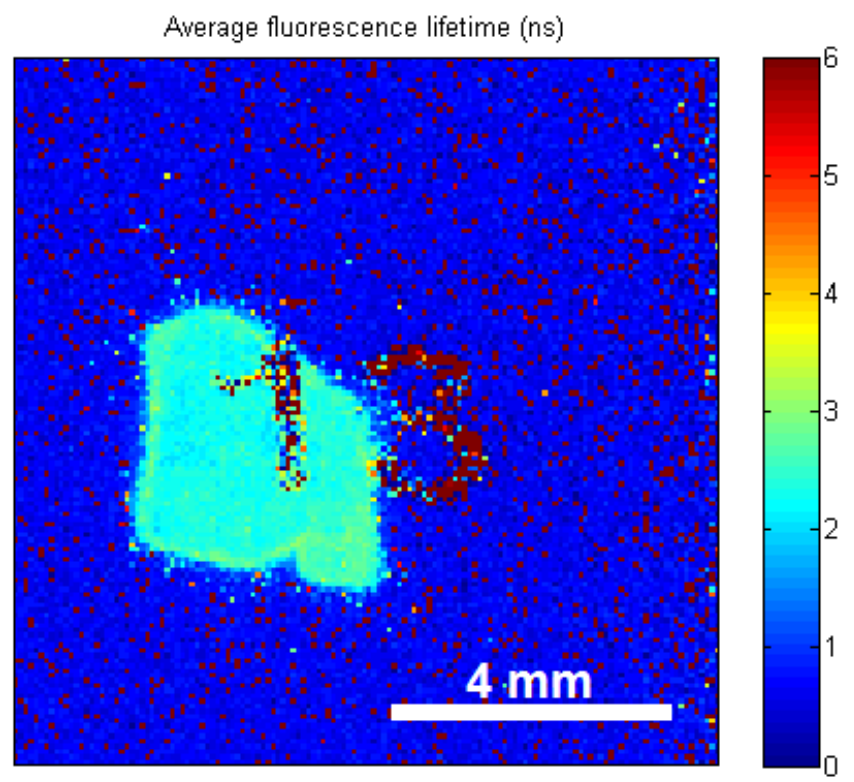

Figure 5.5 - Average lifetime map of the test sample: it is possible to obtain contrast not from the fluorescence intensity, but by the mean fluorescence decay time. Because some regions of the sample present low fluorescence (low signal-to-noise ratio), the quality of the curve fitting is compromised and meaningless fluorescence lifetimes result from the calculations of those points.

Source: By the author.

\subsubsection{IRF characterization}

As the signal of each spectral channel is guided through optical fibers with different lengths, the IRF of each channel is expected to be different due to dispersion effects.

To measure the IRF of the system, we imaged an aluminum coated mirror with the handheld probe. The reflection of the mirror is instantaneous and the measured signal will be identical to the one of a fluorophore with $\tau \rightarrow 0$.

After being reflected by the mirror, a small percentage of the laser light will be transmitted by the dichroic mirror DM1 and coupled into the collection fiber. To measure the IRF of the first channel, the filter F1 was removed to allow the laser reflection by DM2 to go into the fiber S1. To measure the IRF of the second channel, DM2 and F2 were removed to allow the laser to be coupled into S2. To measure the IRF of the third channel, the dichroic mirrors DM2 and DM3 and the filter F3 were removed. For an illustrative guide on the parts labeling, please refer to Figure 4.1 on page 44 .

The IRF of the three channels have FWHM of 1.93 ns, 2.02 ns, and 2.15 ns respectively (Figure 5.7). The IRF of the first channel is wider than the one measured on the on-the-bench setup due to dispersion on the excitation and collection fibers. Using this result, we estimated that the $200 \mu \mathrm{m}$ core fibers introduce a dispersion of $\sim 9.4 \mathrm{ps} / \mathrm{m}$ and the $50 \mu \mathrm{m}$ core excitation fiber introduces a dispersion of $\sim 7.5 \mathrm{ps} / \mathrm{m}$. 


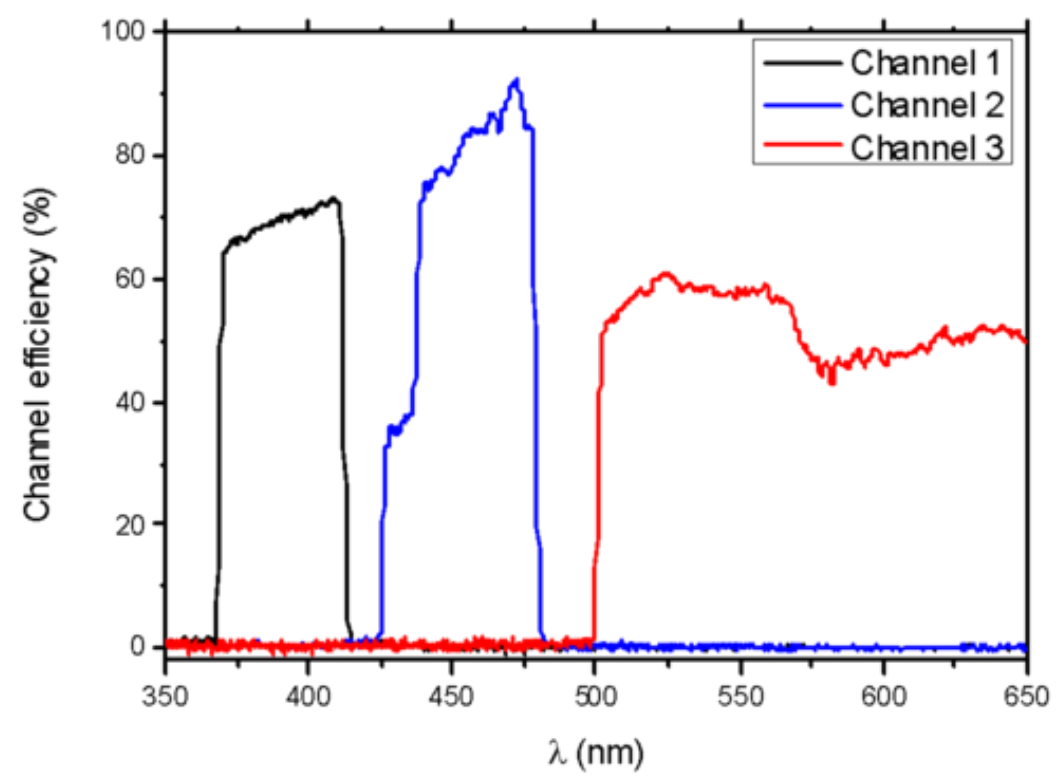

Figure 5.6 - Spectral efficiency of the FLIM channels.

Source: By the author.

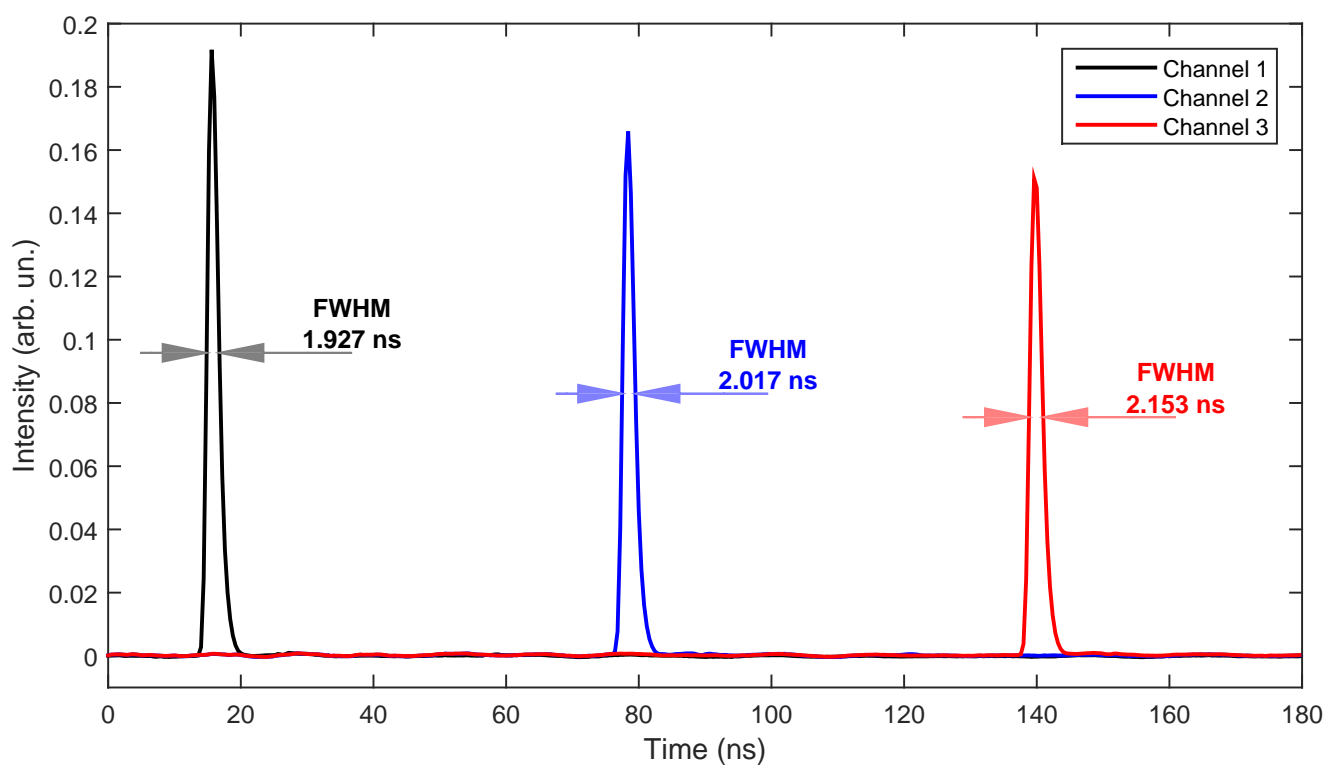

Figure 5.7 - The Instrument Response Functions of the three spectral channels have FWHM of respectively $1.927 \mathrm{~ns}, 2.017 \mathrm{~ns}$, and $2.153 \mathrm{~ns}$. The curves become progressively broader due to dispersion effects introduced by the fibers.

Source: By the author. 


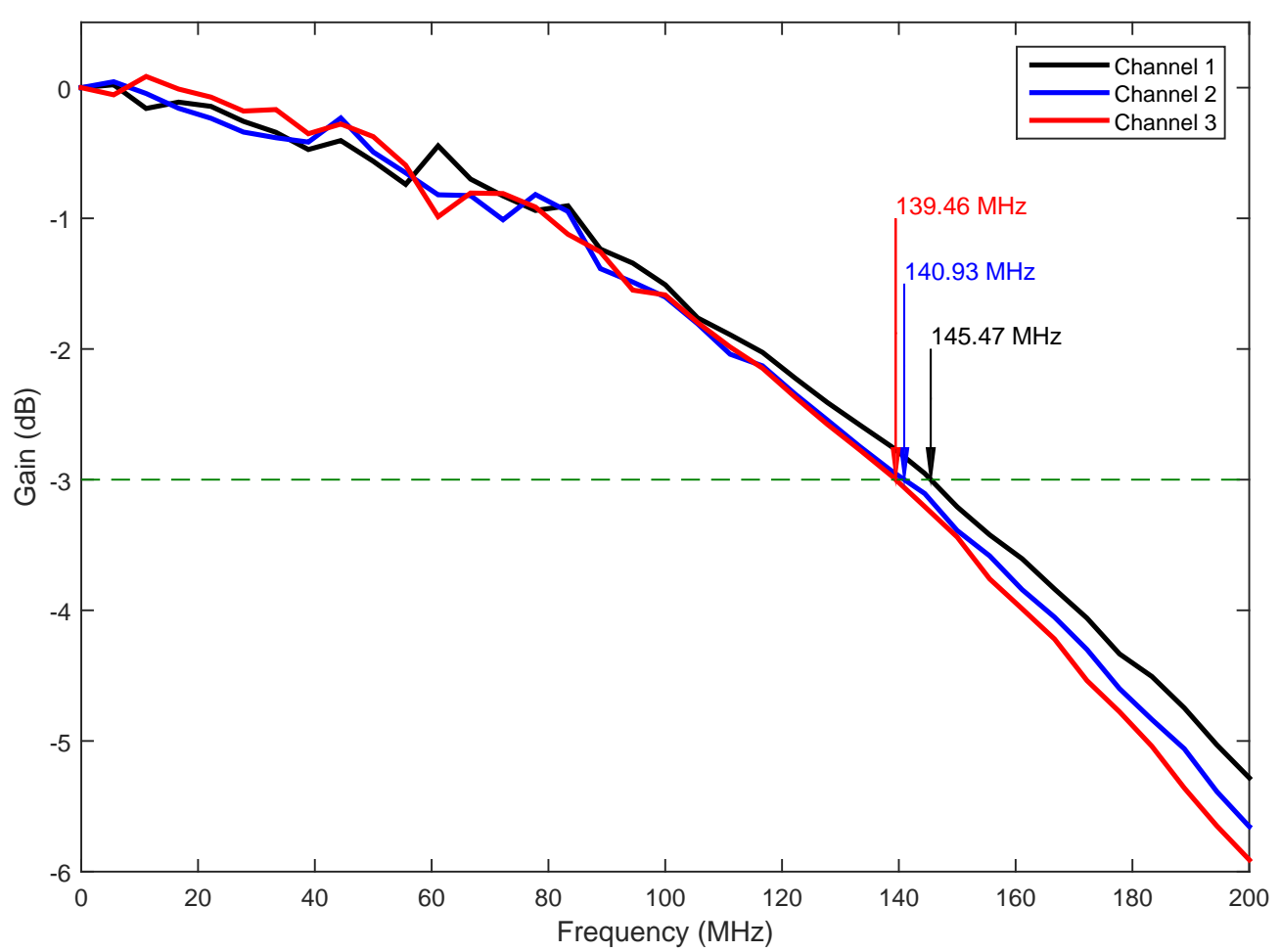

Figure 5.8 - Amplitude of the Transfer function for the three spectral channels. The $-3 d B$ bandwidth decreases slightly from channel to channel due to the dispersion of the fiber, that adds further frequency filtering to the signal.

Source: By the author.

As discussed on Section 3.4, the measured signal will be the result of the convolution of the fluorescence impulse response with the IRF, and this results in a frequency filtering of the fluorescence impulse response. We have seen that the convolution can be written as Equation 3.18, which means that the convolution is equivalent to an amplitude and phase modulation to the Fourier transform of the fluorescence impulse response.

The function modulating the frequency components of the fluorescence impulse response is called Transfer Function, and it is the Fourier transform of the IRF. The $-3 d B$ analog bandwidth of the system can be obtained from the amplitude curve of the Transfer function (Figure 5.8).

The $-3 d B$ frequency threshold for the three spectral channels are $145.47 \mathrm{MHz}$, 140.93 MHz, and 139.46 MHz. The channels with longer fibers present a lower bandwidth because of the increased filtering by broader IRFs.

This result indicates that the reduced bandwidth of the digitizer we assembled with respect to the one reported by Cheng et al. ${ }^{23}$ is not a limiting factor, since the transfer function has a much smaller bandwidth for all three channels. 


\subsubsection{Measurements at the animal model}

In this Subsection, we will present some results obtained at the animal model. The FLIM images were processed and fitted by a biexpoential model, using the method discussed on the Subsection 3.4.2.

Human SCC tumors were induced in BALB/c nude mice by intradermal injection. The animals were imaged right before and right after injection, and every $24 h$ after that for 7 days. Both images acquired right before and right after injection did not show any characteristic features, being composed of basically a flat surface visible on all FLIM parameters.

After $24 h$, the SCC tumor had little growth and presented itself as an approximately $2 \mathrm{~mm}$ nodule on the animal skin (Figure 5.9). The FLIM image of the tumor revealed a significant reduction on fluorescence intensity on the three spectral channels, accompanied by a modification on $a_{1}, a_{2}$ and $\tau_{1}$ on the first channel (Figure 5.10).

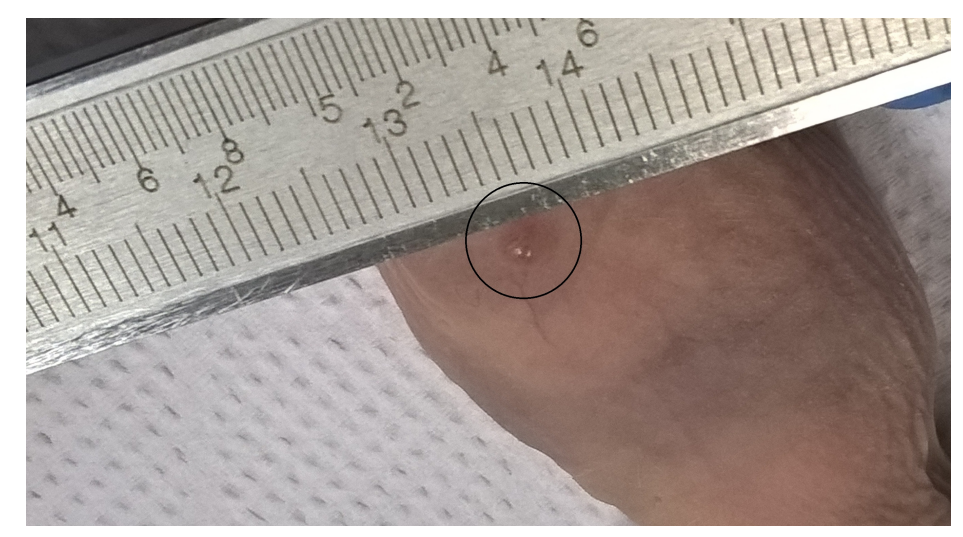

Figure 5.9 - Squamous Cell Carcinoma tumor induced on BALB/c nude mice: $24 h$ after injection.

Source: By the author.

After $168 h$ of tumor growth, the FLIM image shows approximately the same characteristic shown by the image acquired after $24 h$ of tumor growth: a reduction on the fluorescence intensity on all three channels and a slight increase on $a_{1}$, decrease on $a_{2}$ and reduction on $\tau_{1}$ (Figure 5.11). 


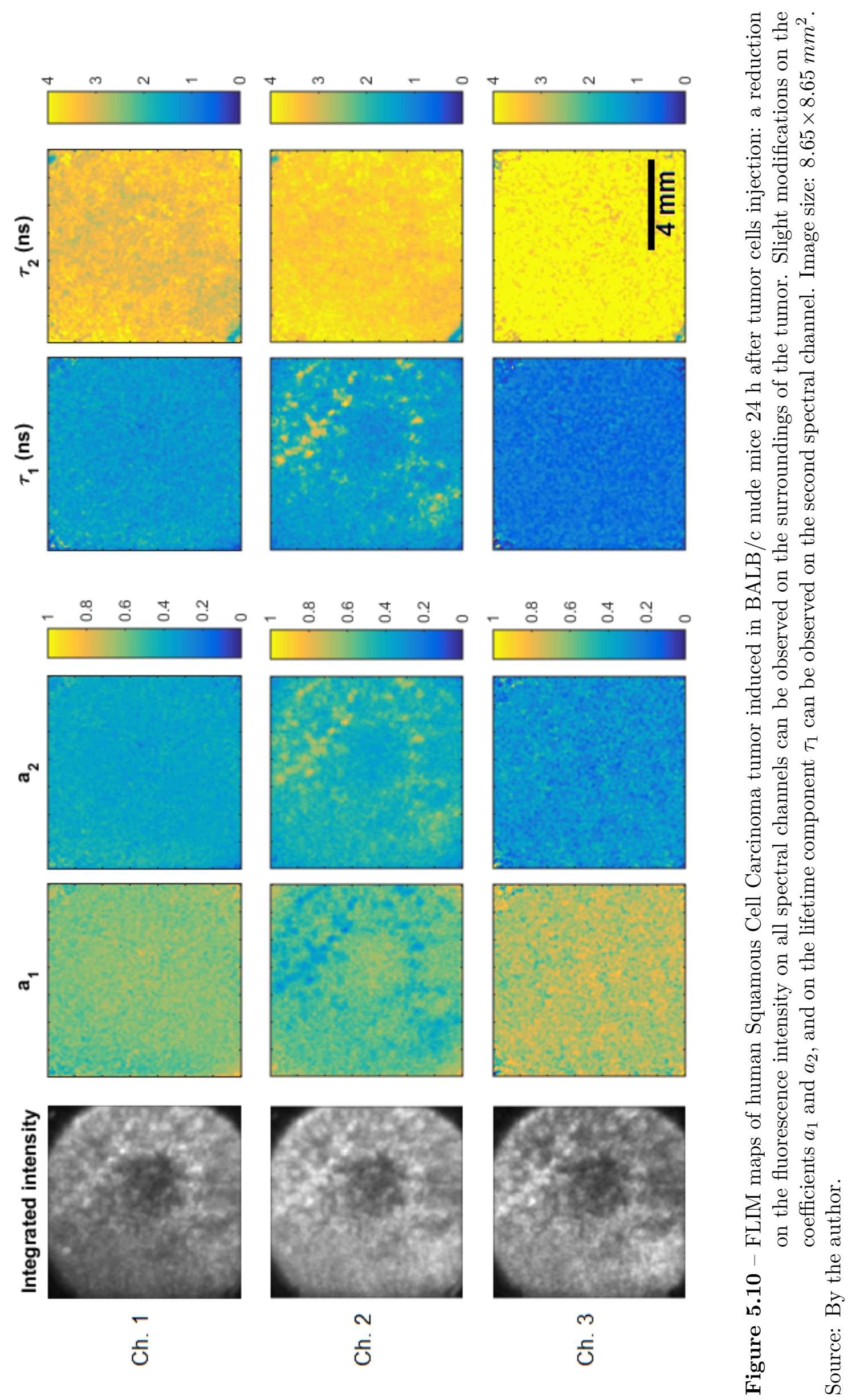




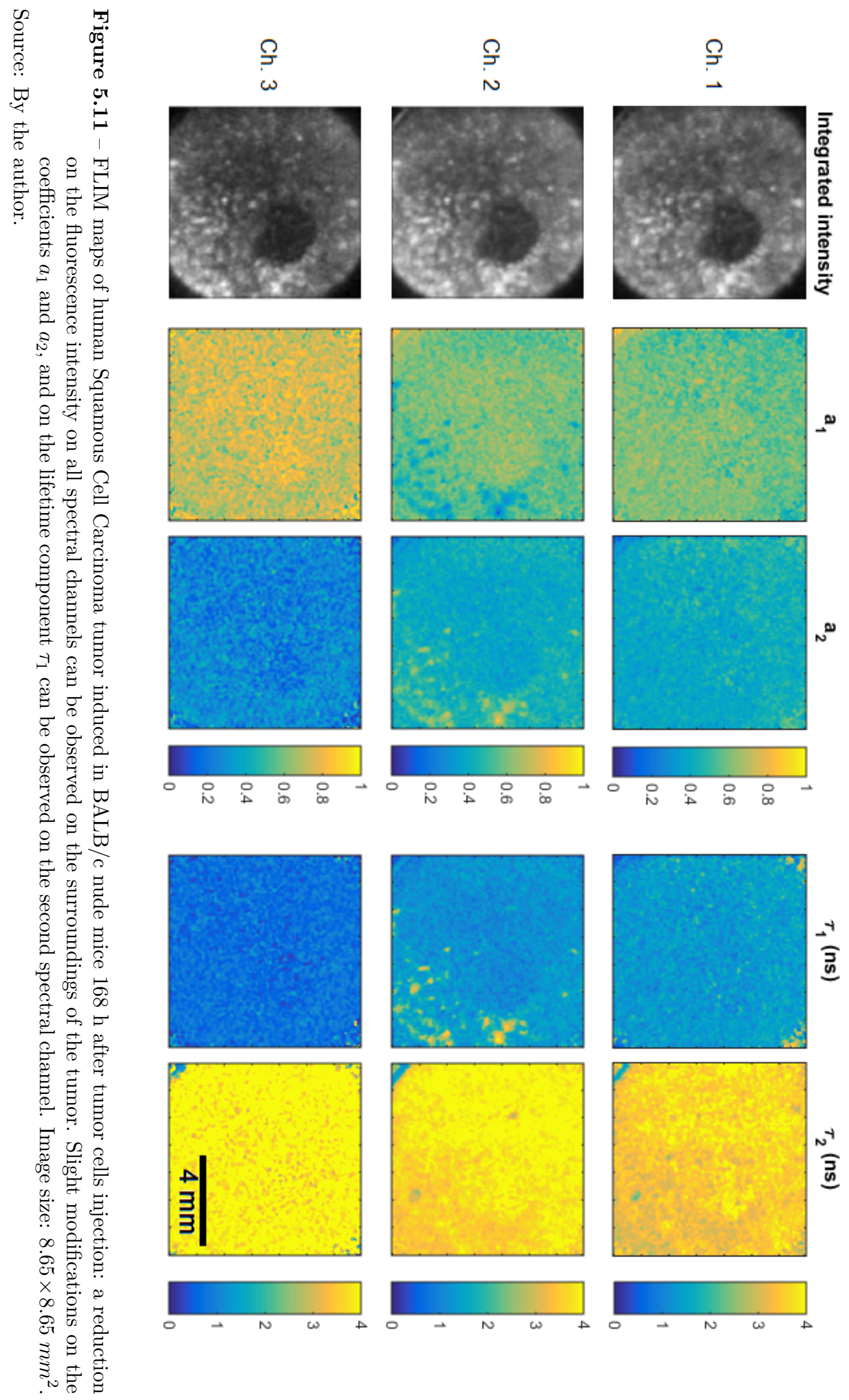


We also induced human melanoma on BALB/c nude mice by intradermal injection. We imaged the animals right before, right after and then every $24 h$.

This type of tumor develops from melanocytes, which are pigmented cells. Thus, a typical characteristic of this tumor is a dark color caused by the high concentration of melanin. The melanin has a strong optical absorption on both excitation and emission wavelengths, what results in the reduction of the fluorescence.

We observed that melanoma has a much faster growth than the induced SCC and is visually much darker. Up to the $8^{\text {th }}$ day after injection, the only recognizable feature on the FLIM images was a darker spot (region with reduced fluorescence spectrum on all three channels). On the $9^{\text {th }}$ day, we observed modifications on the top layer of skin (Figure 5.12) and it was possible to observe slight modifications on the coefficients $a_{1}$ and $a_{2}$, and a significant reduction on $\tau_{2}$ of the first channel. (Figure 5.13).

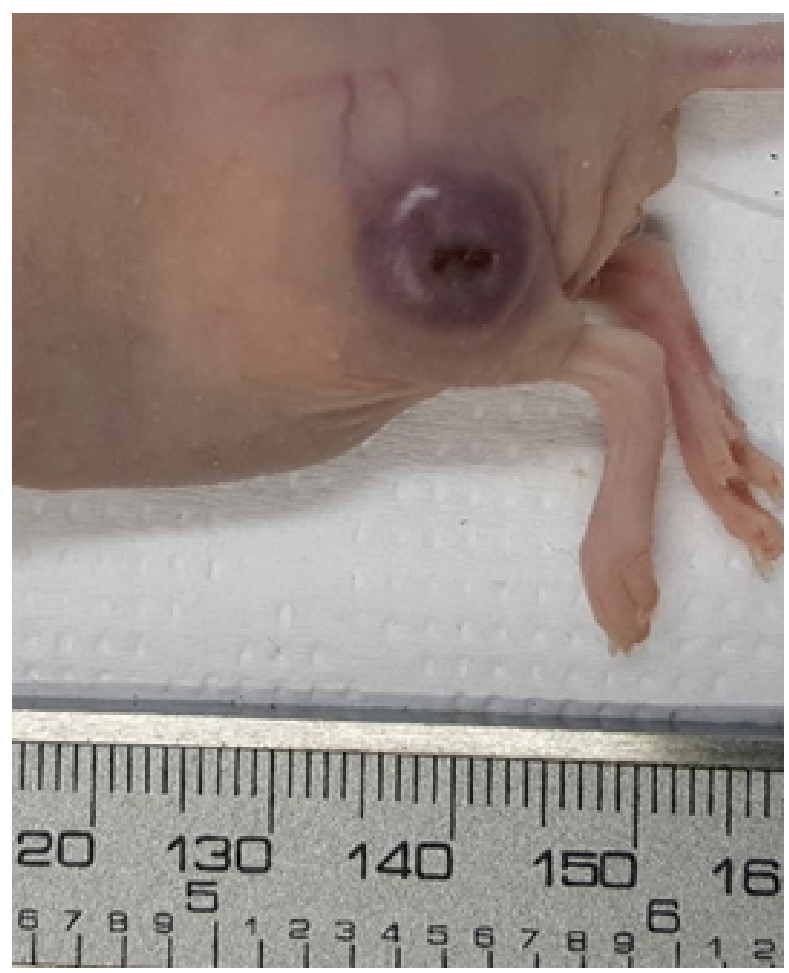

Figure 5.12 - Melanoma induced on BALB/c nude mice: 9 days after injection. At this point, modifications on the surface of skin were observed.

Source: By the author.

We believe the smaller area where a modification on the lifetime components was observed is the region in which the tumor is ulcerated. As it the tumor cells were injected into the dermis, the first stages of tumor growth happen under the surface of skin. The penetration of the $355 \mathrm{~nm}$ excitation is rather short in skin due to absorption and scattering, so we hypothesize that we are only being able to detect the cells with modified metabolism when the tumor growth reaches shallower layers of the skin. 


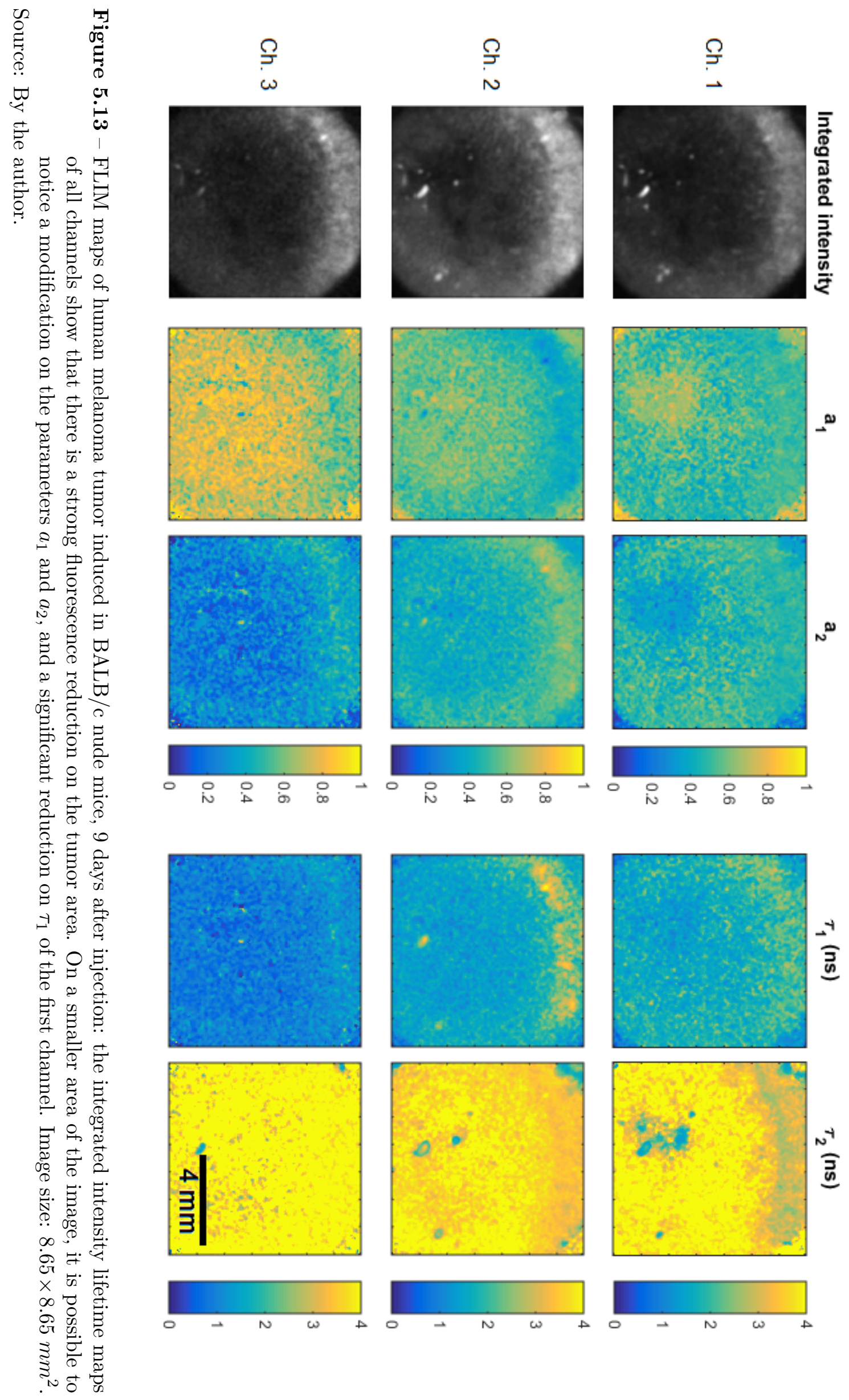




\subsubsection{Validation with human skin imaging}

We imaged a nonneoplastic lesion produced by a small skin inflammation on the forearm of a volunteer. A photograph of the lesion is shown on Figure 5.14.

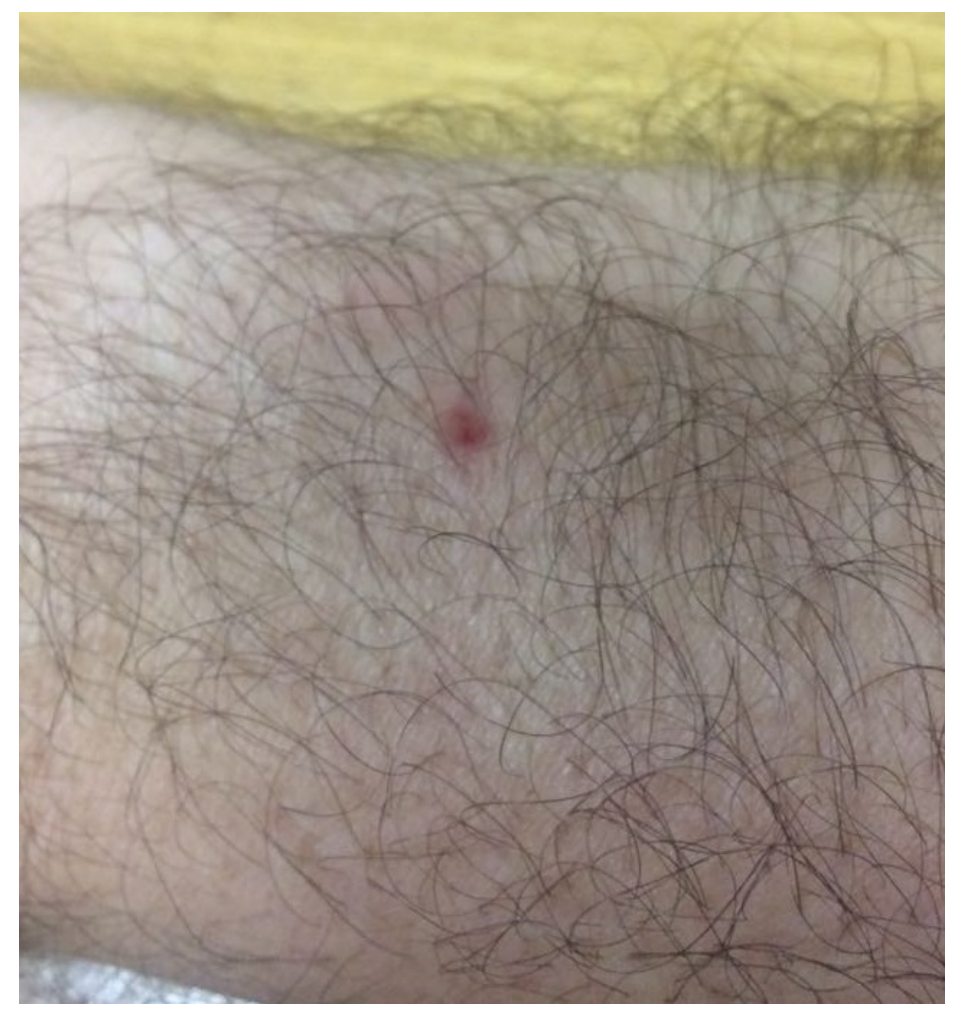

Figure 5.14 - Nonneoplastic lesion produced by a small skin inflammation. This lesion was imaged with the assembled FLIM system. The FLIM images are shown on Figures 5.15 e 5.16 .

Source: By the author.

The FLIM image was acquired and the data was analyzed by both the modified phasor approach (Equation 3.23) and by a biexponential deconvolution model. The results are shown on Figures 5.15 e 5.16, respectively.

This result, besides being a clinically unimportant lesion, clearly illustrates the importance of FLIM or other time-resolved fluorescence based techniques. The integrated intensity maps fail to provide useful contrast to locate the lesion or to identify its borders. However, the maps generated using the dynamics information contained on the timeresolved data can clearly reveal the lesion and its borders. 


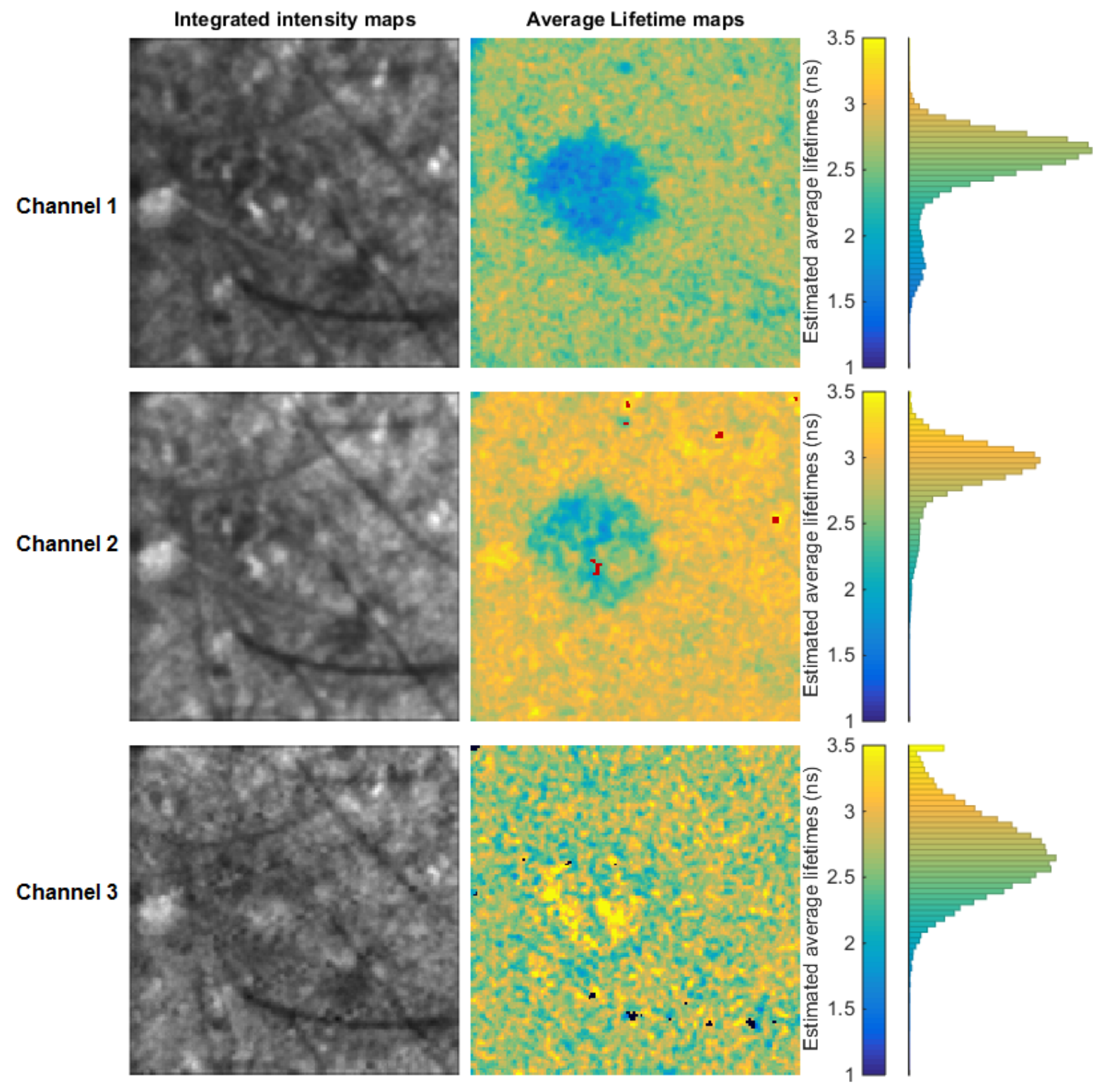

Figure 5.15 - FLIM maps of a nonneoplasic lesion produced by a small skin inflammation. The FLIM maps were generated using the modified phasor approach. The lesion is clearly visible on the average lifetime maps of channels 1 and 2 , but is not visible on the integrated intensity maps. Image size: $8.65 \times 8.65 \mathrm{~mm}^{2}$.

Source: By the author. 


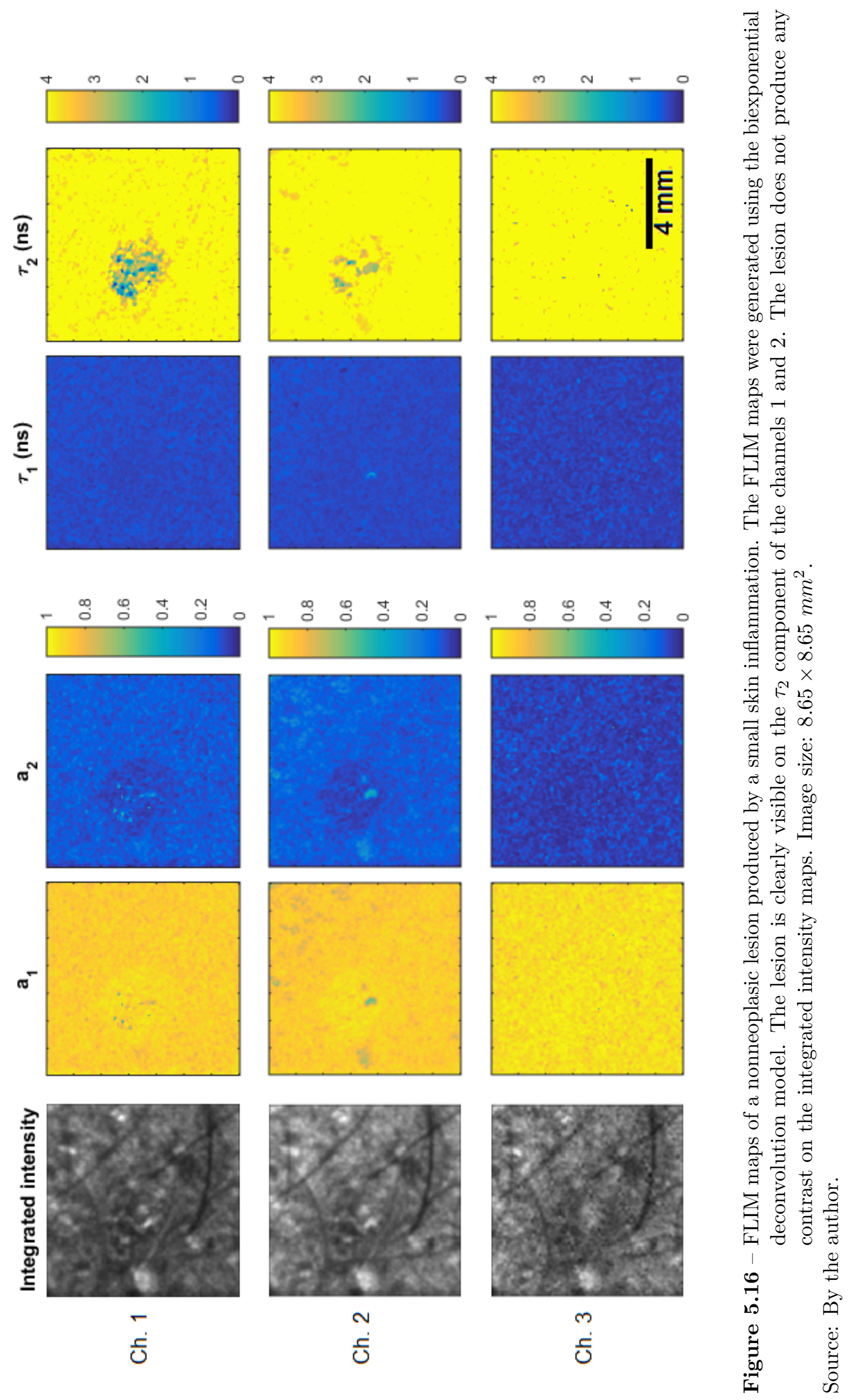




\subsection{Clinical study}

The human tests were performed on voluntary patients, with varying ages, of both sexes.

We imaged a Bowen's disease lesion. This tumor has a light red color (Figure 5.17). Figure 5.18 shows the FLIM maps acquired from a Bowen's disease. This tumor presented a much lower fluorescence intensity than the surrouding healthy areas, as can be seen on the integrated intensity maps. The tumor also produces significant changes on all the other parameters. The longer lifetime component $\left(\tau_{2}\right)$ also shows some structures internal to the tumor. These kinds of structures may be useful for identifying this kind of lesion.

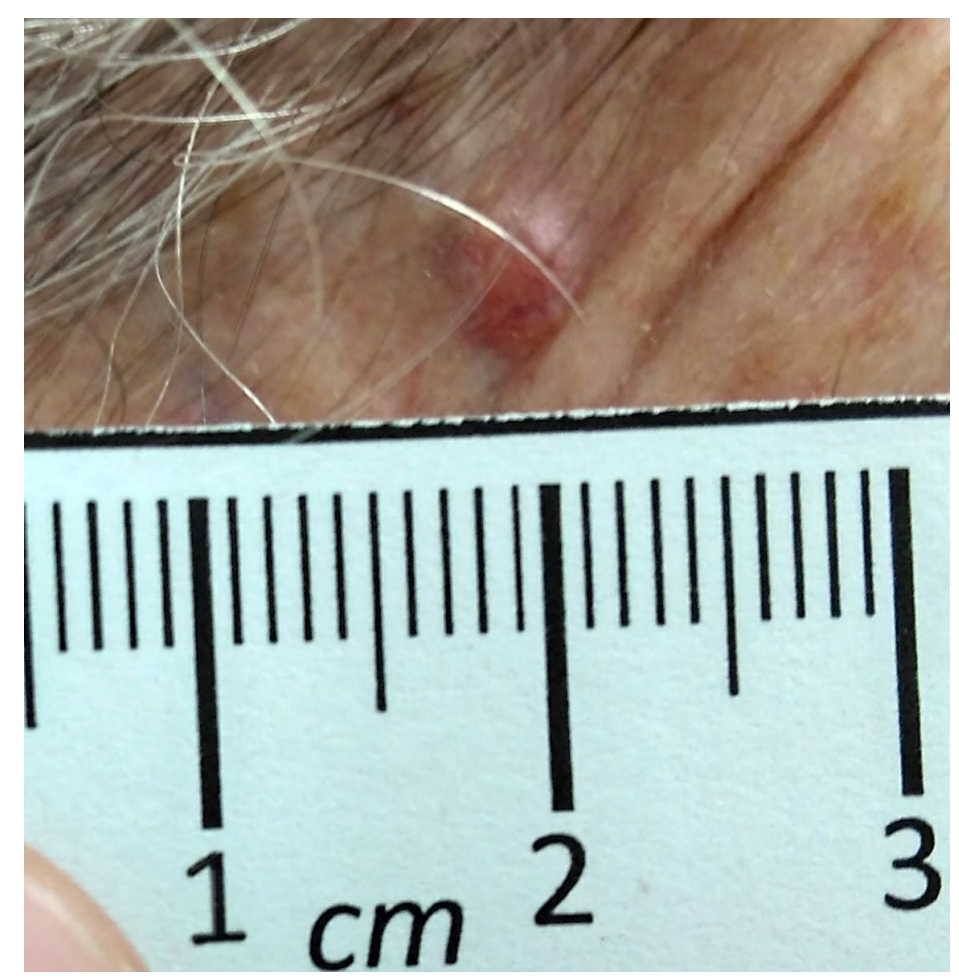

Figure 5.17 - Picture of the Bowen's disease.

Source: By the author.

We also imaged a melanoma tumor. As previously stated, this kind of lesion features a dark color and lower fluorescence. The area with reduced fluorescence can be observed on the integrated intensity maps, but no significant modifications were observed on the other coefficients and lifetimes (Figure 5.19).

We also imaged a seborrheic keratosis lesion, a benign condition, and analyzed the data using the biexpoential deconvolution model. The results are shown on Figure 5.20.

The borders of the seborrheic keratosis can be easily identified on all integrated intensity maps and on the average lifetime of the first spectral channel, for the image processed through the modified phasor approach method. The image processed by the 

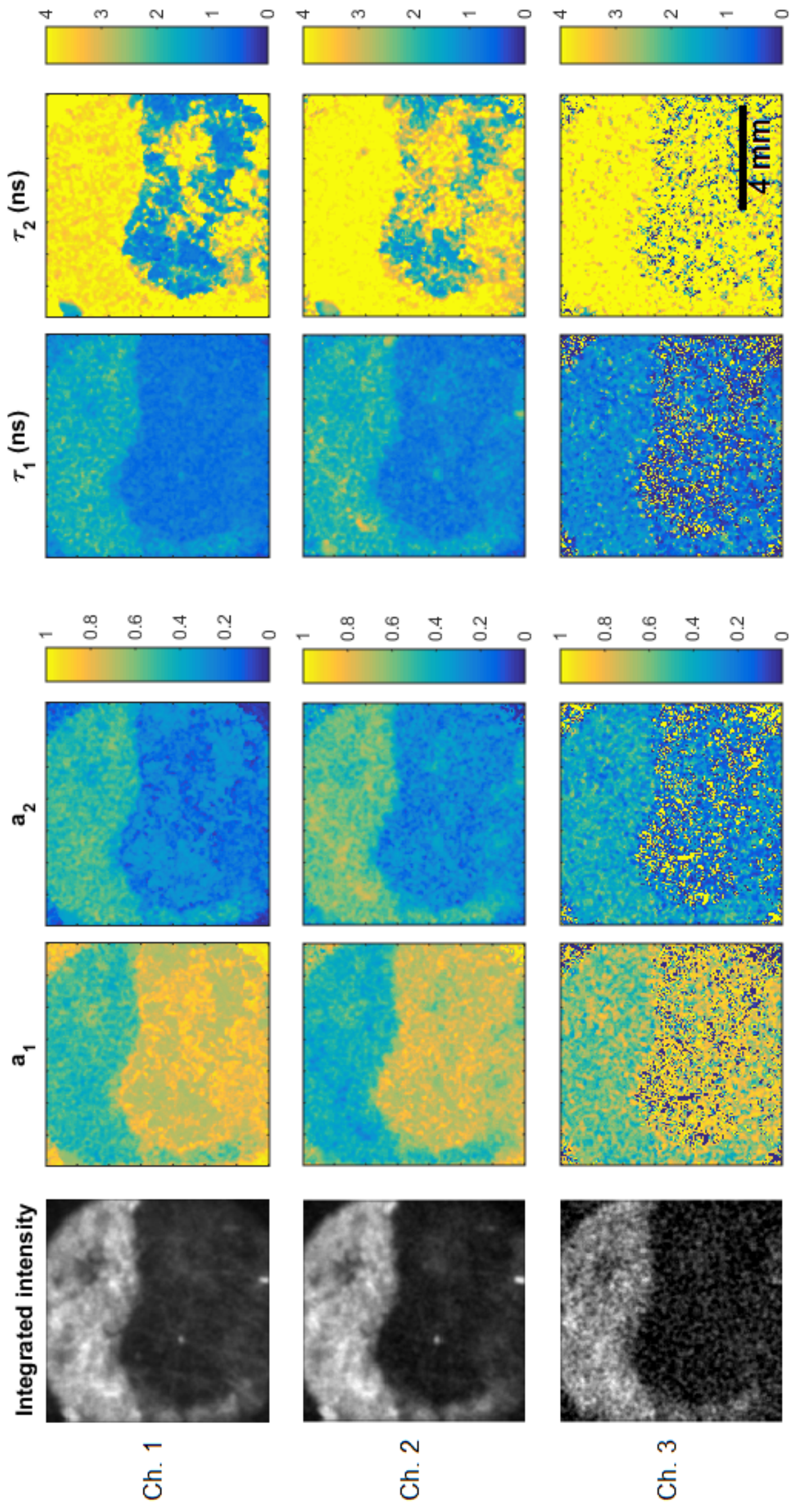

$$
\text { ํ. }
$$
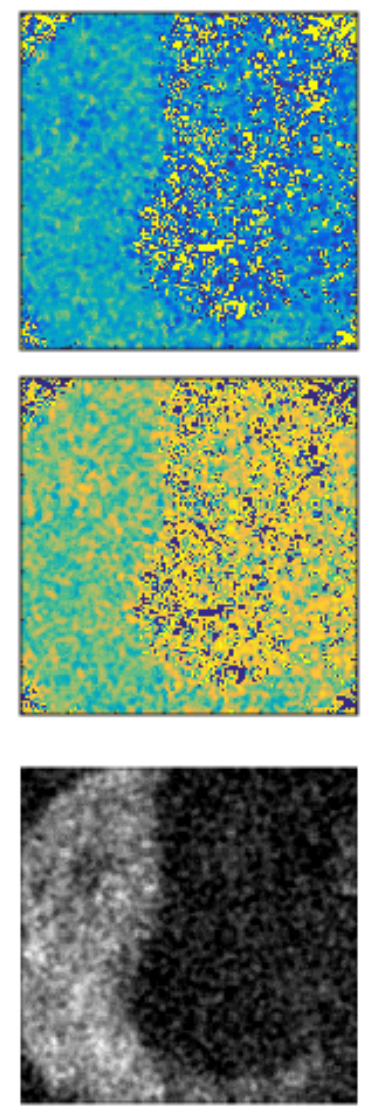

ल

$\begin{array}{cc}0 & 0 \\ 0 & 0 \\ 0 & 0 \\ 0 & 0 \\ 0 & 0 \\ 0 & 0 \\ 0 & 0\end{array}$

苛 $\frac{0}{\sigma}$

疍

\begin{tabular}{ll}
$\dot{0}$ & 0 \\
0 & 0 \\
0 & 0 \\
0 & 0 \\
\hline
\end{tabular}

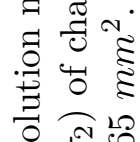

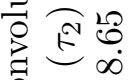

导

.

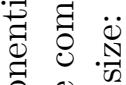

둘 \&

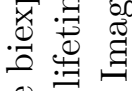

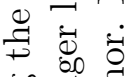

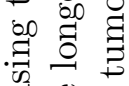

马े

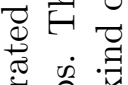

苞

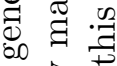

吾

3 这

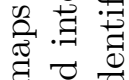

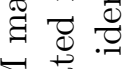

击

届高

电焉

0.0

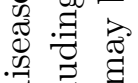

is

궁

है

๑

$\approx=\frac{0}{0}$

낭 శี

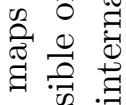

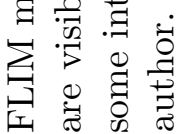

$\underset{1}{\infty}$

10 की

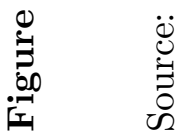


biexponential deconvolution model shows that the main modificaiton on the lifetimes happens on the longer lifetime component $\left(\tau_{2}\right)$ of the first spectral channel.

Alike the melanoma, the dysplastic nevi present dark color and low fluorescence intensity. Figure 5.21 shows the FLIM maps obtained from a dysplastic nevus. The lesion can be clearly seen on the integrated intensity maps. Some structures are seen on the tumor region on the parameters $a_{1}, a_{2}, \tau_{1}$, and $\tau_{2}$, for all the channels (especially channels 1 and 2), but we verified those are artifacts caused by the extremely low fluorescence intensity and consequent low signal-to-noise ratio inside the tumor region, which leads to big fitting error.

We also imaged Nodular and Superficial BCCs (Figures 5.22 and 5.23, respectively). The Nodular BCC presented a reduced fluorescence intensity, but did not change the fluorescence dynamics parameters. The Superficial BCC did not have any contrast on any of the maps of the FLIM image.

We also imaged a Sclerodermiform BCC. The determination of the borders of this kind of tumor stands a challenge because it presents very subtle visual characteristics. The Figure 5.24 shows a picture of the Sclerodermiform BCC we imaged. The lesion is located right below a hypopigmented area.

The FLIM image of this lesion (Figure 5.25 shows an increase on the fluorescence lifetime on the hypopigmented area. The region with higher fluorescence intensity partially surrounds the tumor, with lower fluorescence intensity, making it stand out on this map. We believe if it were not for the hypopigmented area, the tumor would not have been identifiable on the integrated intensity maps.

However, other components, especially the $\tau_{2}$ component of the first spectral channel, give undubious contrast to the tumor. 


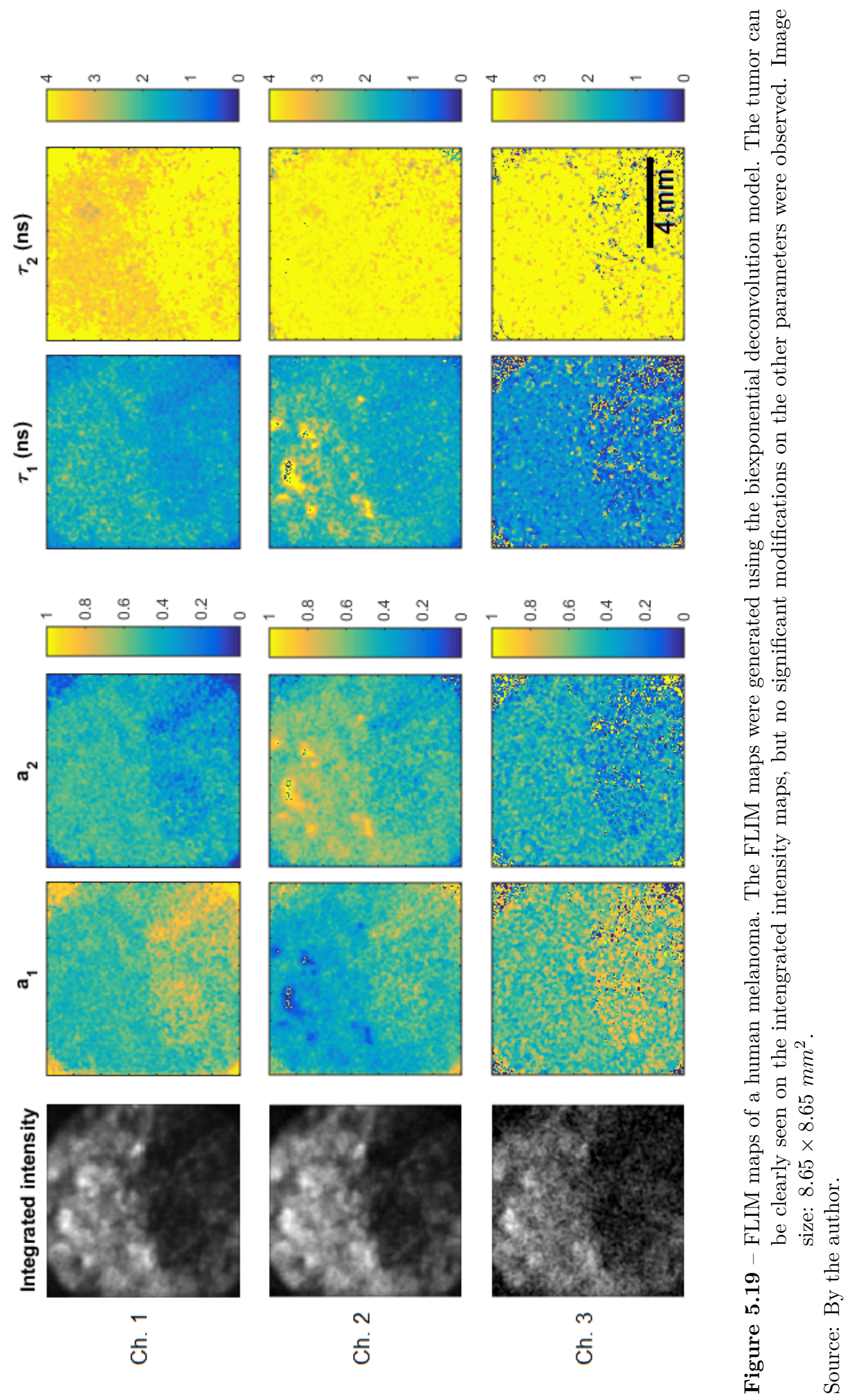




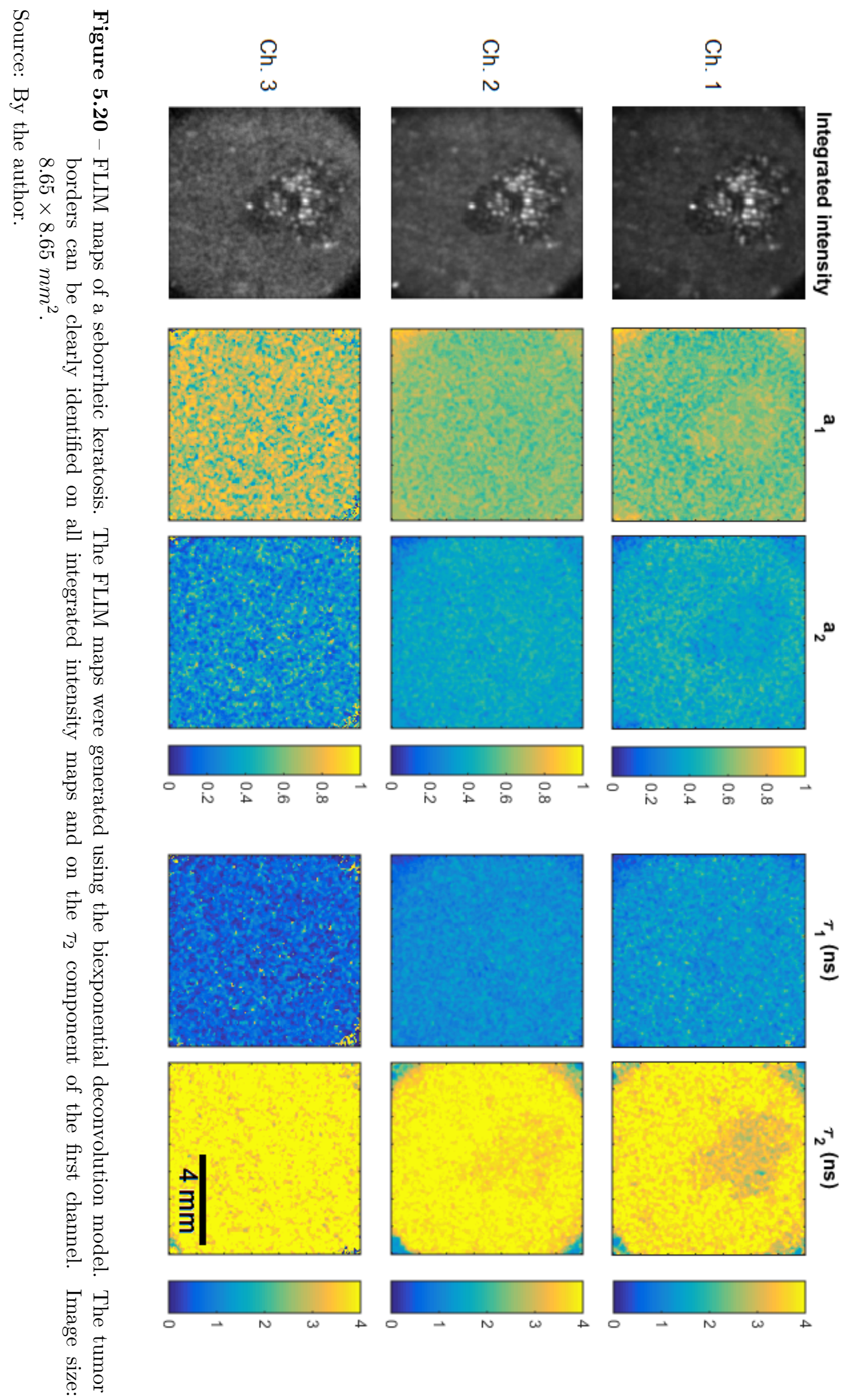



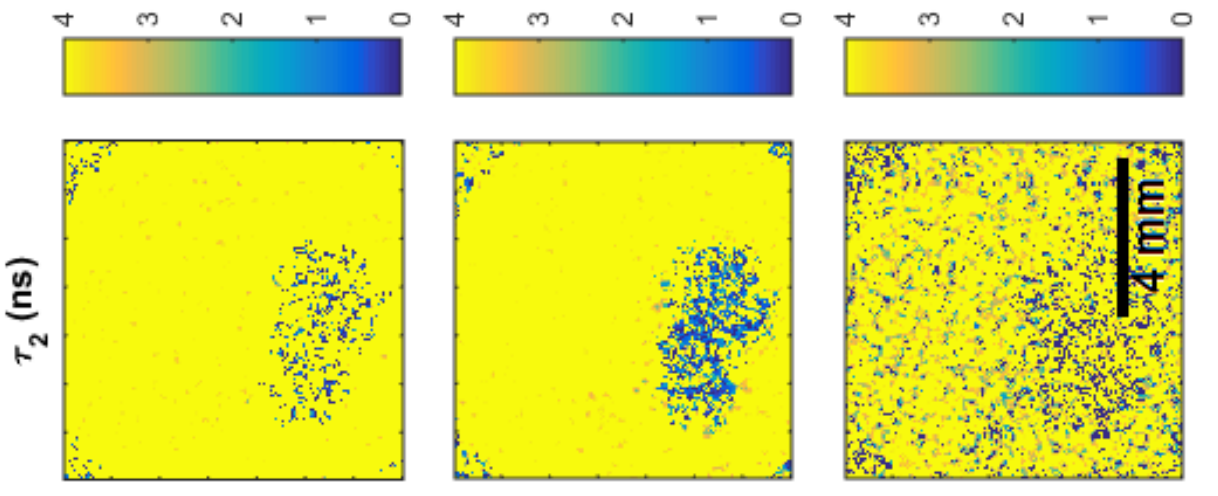

㺃

.ำ

$\stackrel{0}{0}$

ज

सं

द्व

호 :

:

เิ
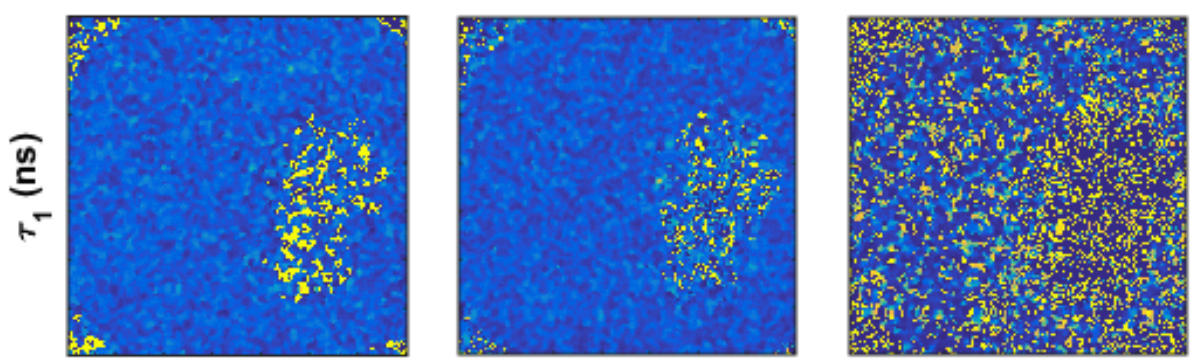

ठํ

.

च्ष

这.

.

ఫ छ छ

吕
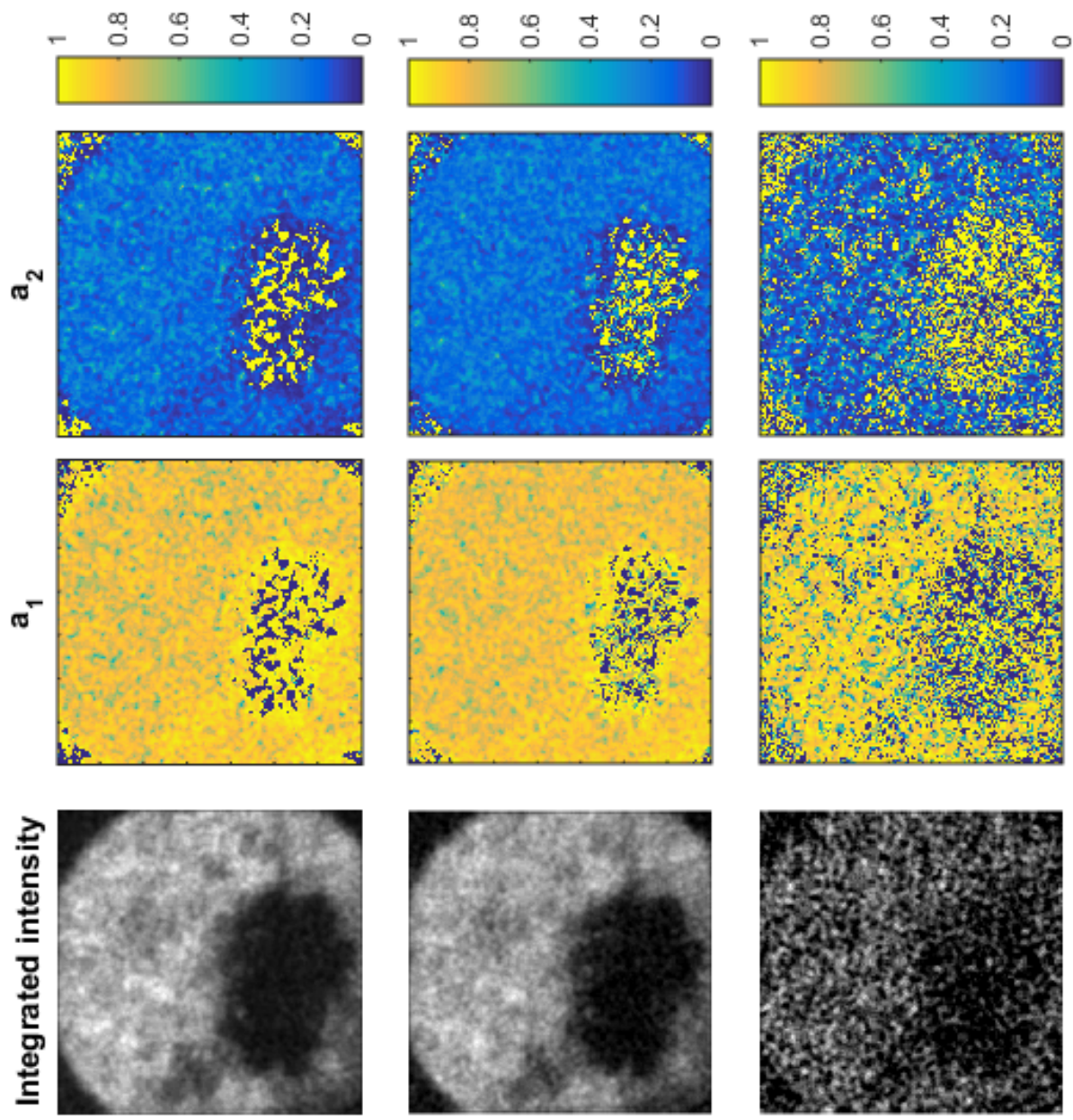

ธ่

$\frac{N}{U}$

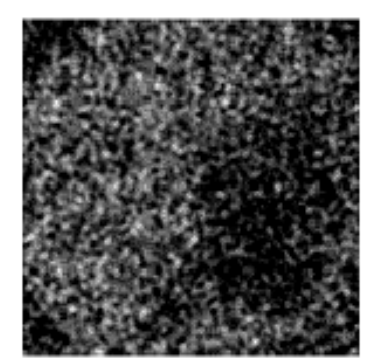

ㄷํㄴ

昰

芯 శ

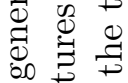

웡

语。

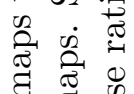

घ $\cong$

空总

可.

.

通贾

ठ

. 불

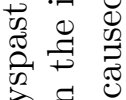

突

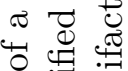

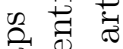

茗.

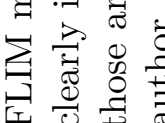

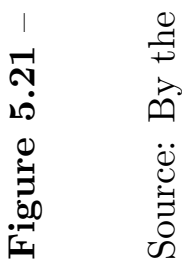




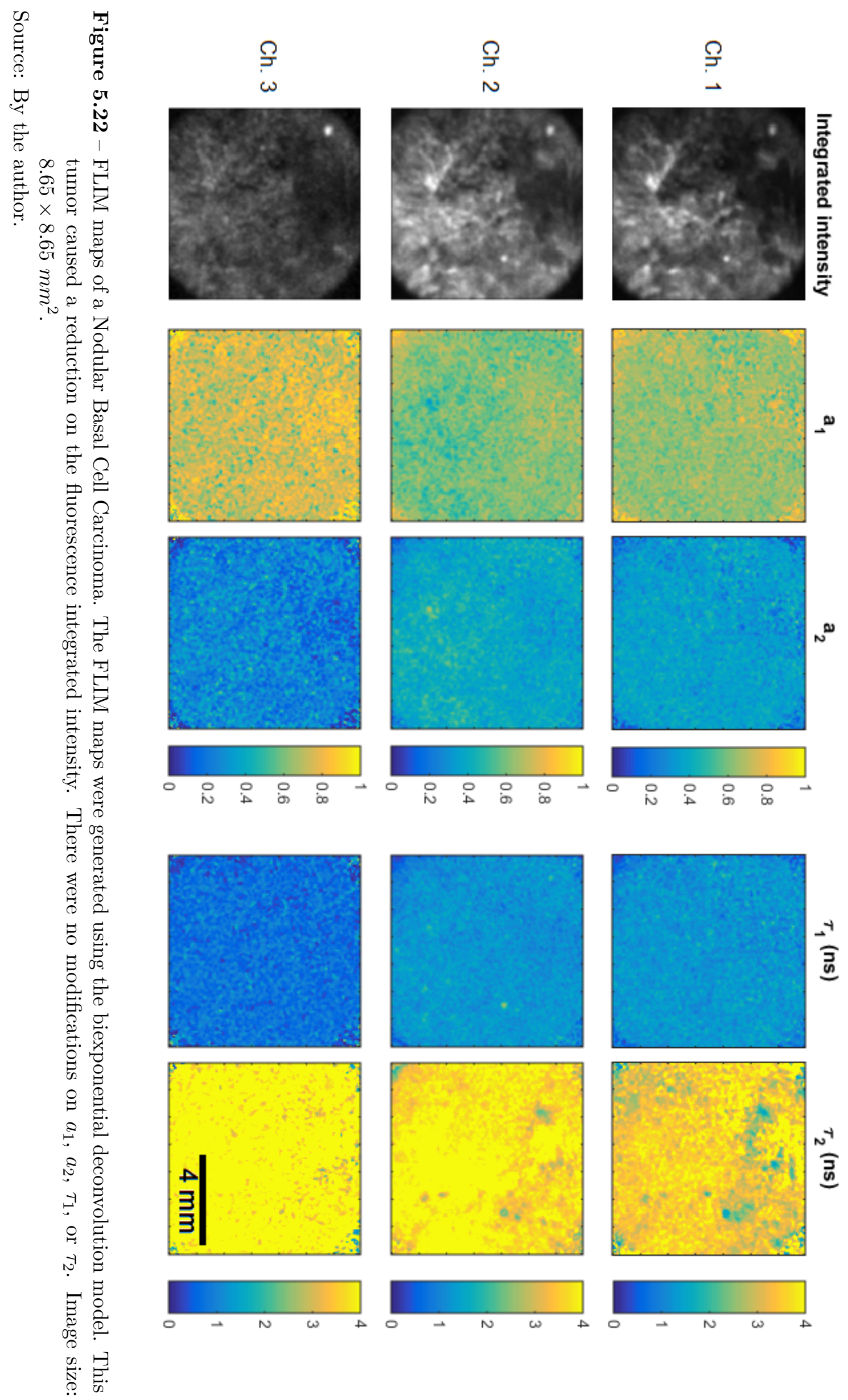




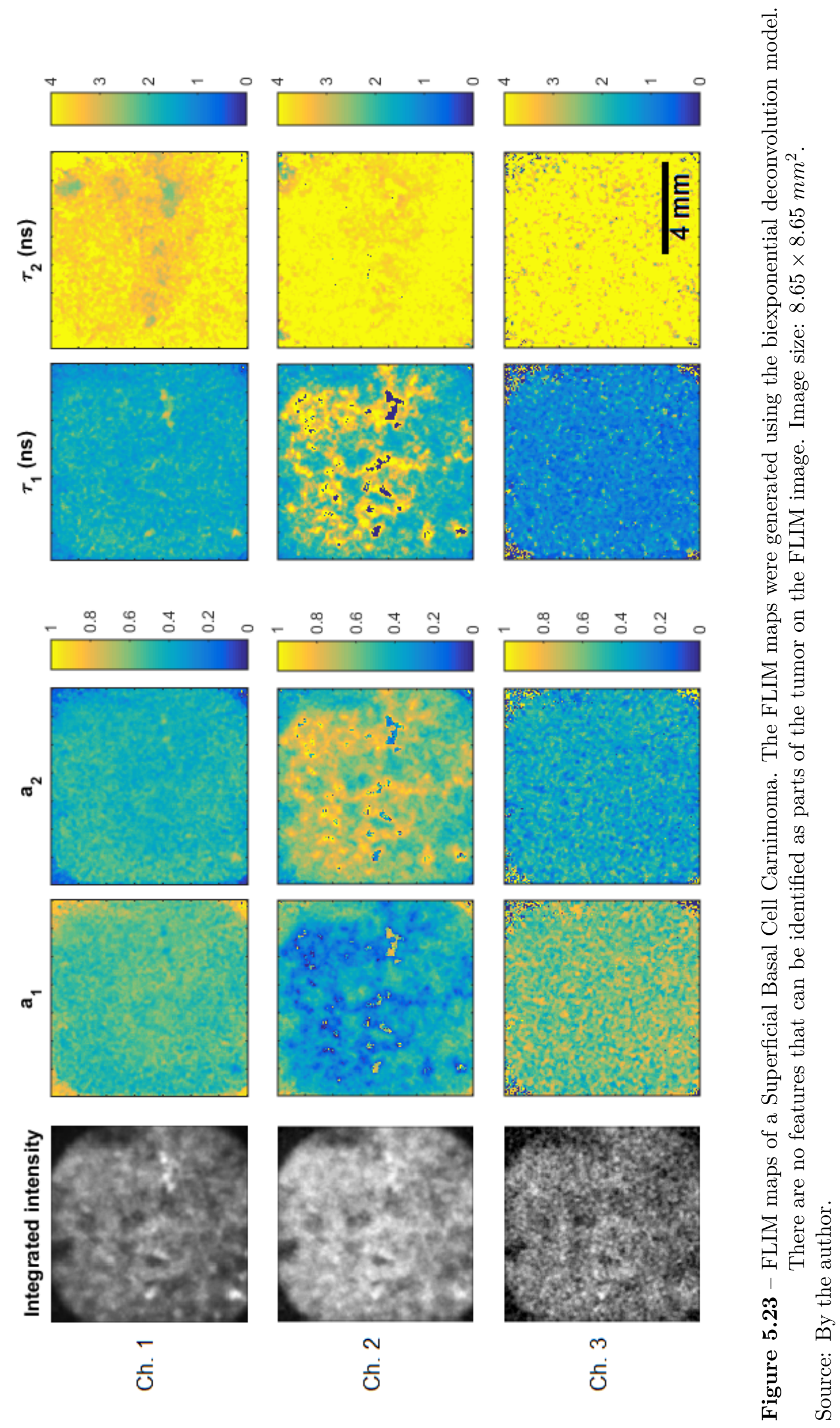




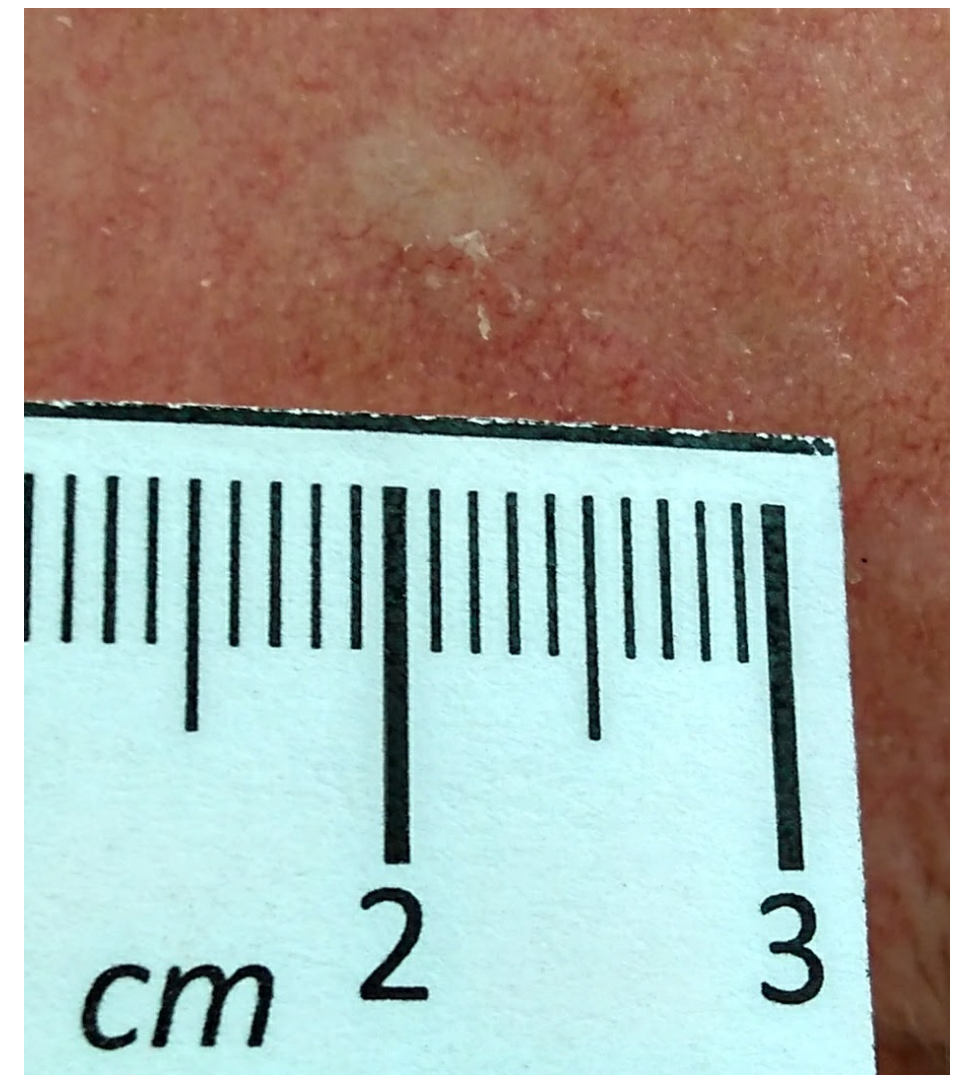

Figure 5.24 - Sclerodermiform Basal Cell Carcinoma: the lesion presents subtle visual characteristics, making the clinical determination of its borders a challenge. The lesion is located right below a hypopigmented area.

Source: By the author. 

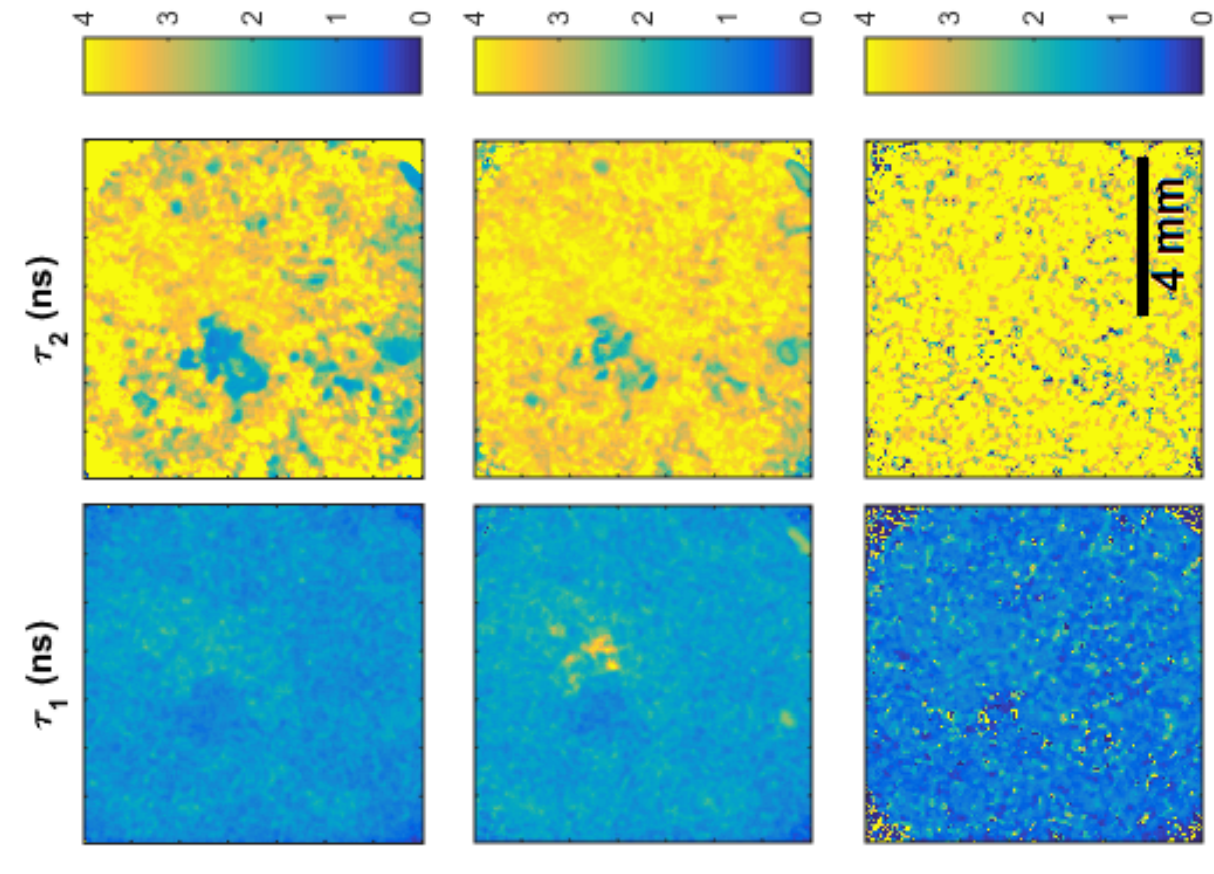

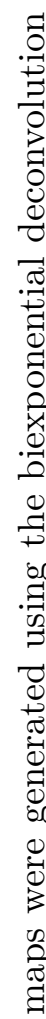
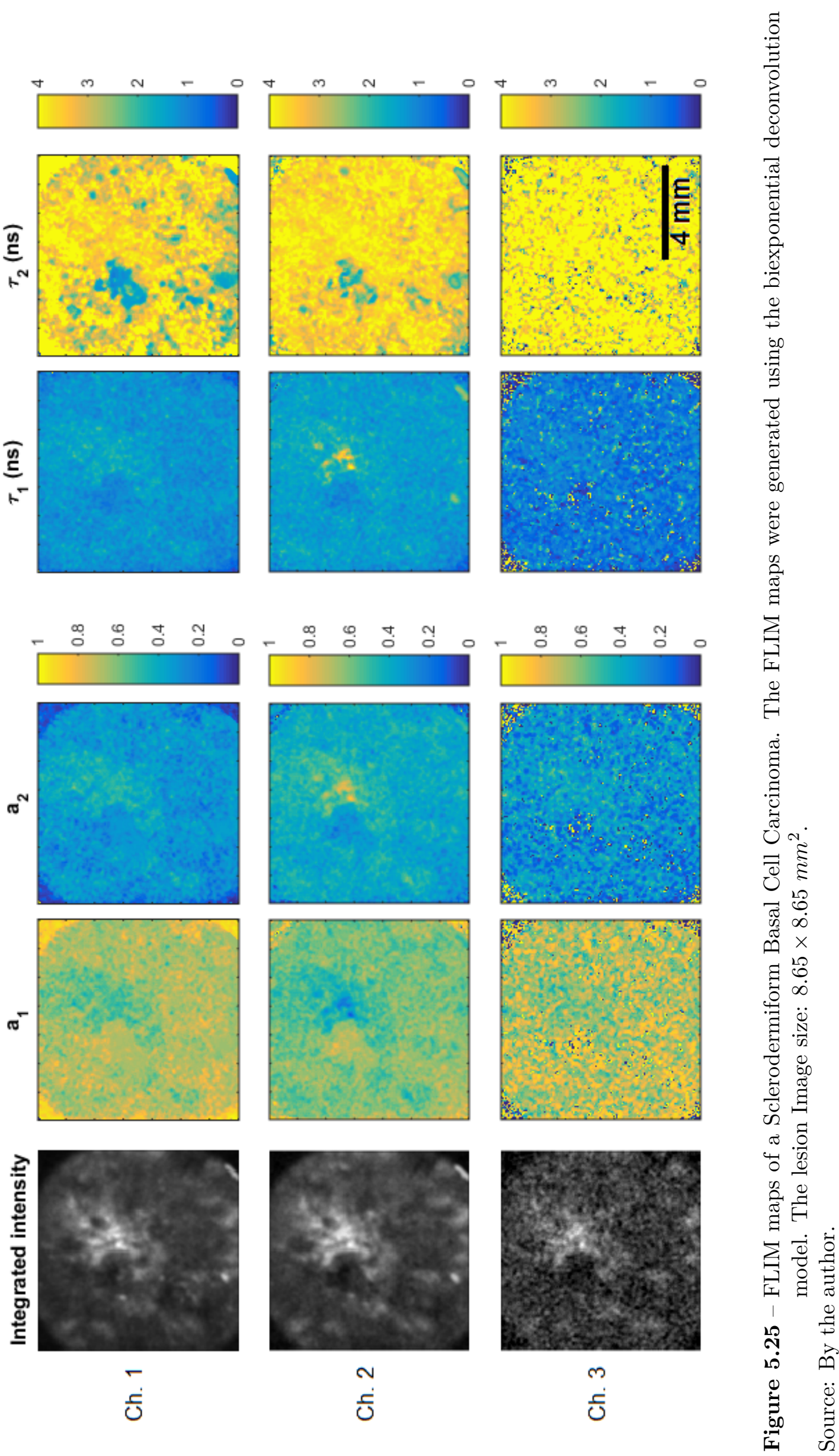
This pilot study confirmed that the assembled FLIM system is suitable for clinical in vivo imaging of skin lesions. We plan to expand this study, measuring multiple lesions of each type for further statistical analysis aiming the discrimination of clinically similar lesions with help of classification algorithms. We also aim the implementation of a realtime pixel-by-pixel classification algorithm to allow the fast and accurate determination of lesion borders.

This research project was submitted to Conselho Nacional de Ética em Pesquisas (CONEP, National Council on Ethics in Research), Brazil. It received a positive opinion from the local research ethics committee (Hospital Amaral Carvalho - Jahu, SP, Brazil), but the review from CONEP is still pending. 


\section{Conclusions}

We assembled a fast multispectral FLIM system suitable for in vivo applications. The system features a handheld probe consisting of a rigid laser scanning endoscope.

The FLIM system records $140 \times 140$ pixels images with FOV of $8.65 \times 8.65 \mathrm{~mm}^{2}$ in $\sim 2.4 s$ using a single laser pulse per pixel, operating at $10 \mathrm{kHz}$ with average power at the sample of $10 \mathrm{~mW}$.

The system was validated with in vivo measurements of skin lesions at an animal model. Both SCC and melanoma models produced none to little contrast on the lifetime maps before ulceration. The ulcerated region of the melanoma tumor was clearly detected on the lifetime maps.

We performed a pilot clinical study to evaluate the system performance for in vivo imaging of skin lesions and presented here the results of seven types of lesions. From those lesions, a significant constrast was achieved on the imaging of seborrheic keratosis, Bowen's disease and sclerodermiform BCC.

An expanded study is necessary to evaluate the statistical significance of our results. With the imaging of an increased number of lesions, trained classifications algorithms

may be able to produce binary maps of malignant lesions or to discriminate between clinically similar lesions. 


\section{References}

1 WORLD HEALTH ORGANIZATION. Cancer. Available from: 〈http://www. who. int/mediacentre/factsheets/fs297/en/>. Accessible at: 13 Nov. 2017.

2 WAGNIERES, G. A.; STAR, W. M.; WILSON, B. C. In vivo fluorescence spectroscopy and imaging for oncological applications. Photochemistry and photobiology, v. 68, n. 5, p. 603-632, 1998.

3 MIZERET, J. et al. Instrumentation for real-time fluorescence lifetime imaging in endoscopy. Review of Scientific Instruments, v. 70, n. 12, p. 4689-4701, 1999.

4 LAKOWICZ, J. Principles of fluorescence spectroscopy. 3rd ed. New York: Springer, 2006.

5 ACUNA, A. U. et al. Structure and formation of the fluorescent compound of lignum nephriticum. Organic Letters, v. 11, n. 14, p. 3020-3023, 2009.

6 ROST, F. W. Fluorescence microscopy. Cambridge: Cambridge University Press, 1995. v. 2 .

7 MARCU, L. Fluorescence lifetime techniques in medical applications. Annals of Biomedical Engineering, v. 40, n. 2, p. 304-331, 2012.

8 BEREZIN, M. Y.; ACHILEFU, S. Fluorescence lifetime measurements and biological imaging. Chemical Reviews, v. 110, n. 5, p. 2641-2684, 2010.

9 CHEN, Y.; PERIASAMY, A. Characterization of two-photon excitation fluorescence lifetime imaging microscopy for protein localization. Microscopy Research and Technique, v. 63, n. 1 , p. $72-80,2004$.

10 MYCEK, M.-A.; POGUE, B. W. Handbook of biomedical fluorescence. New York: CRC Press, 2003.

11 CHORVAT, D.; CHORVATOVA, A. Multi-wavelength fluorescence lifetime spectroscopy: a new approach to the study of endogenous fluorescence in living cells and tissues. Laser Physics Letters, v. 6, n. 3, p. 175-193, 2009. 
12 EBENEZAR, J. et al. Diagnostic potential of stokes shift spectroscopy of breast and prostate tissues - a preliminary pilot study. Technology in Cancer Research \& Treatment, v. 10, n. 2, p. 153-161, 2011.

13 FRUHWIRTH, G. O. et al. Fluorescence lifetime endoscopy using tcspc for the measurement of fret in live cells. Optics Express, v. 18, n. 11, p. 11148-11158, 2010.

14 KENNEDY, G. T. et al. A fluorescence lifetime imaging scanning confocal endomicroscope. Journal of Biophotonics, v. 3, n. 1-2, p. 103-107, 2010.

15 KENNEDY, G. et al. Fluorescence lifetime imaging endoscopy. Proceedings of the SPIE, v. 7893, p. 789308, 2011. doi: 10.1117/12.874968.

16 MUNRO, I. et al. Toward the clinical application of time-domain fluorescence lifetime imaging. Journal of Biomedical Optics, v. 10, n. 5, p. 051403-051403, 2005.

17 REQUEJO-ISIDRO, J. et al. High-speed wide-field time-gated endoscopic fluorescence-lifetime imaging. Optics Letters, v. 29, n. 19, p. 2249-2251, 2004.

18 ELSON, D.; JO, J.; MARCU, L. Miniaturized side-viewing imaging probe for fluorescence lifetime imaging (flim): validation with fluorescence dyes, tissue structural proteins and tissue specimens. New Journal of Physics, v. 9, n. 5, p. 127, 2007.

19 CHENG, S. et al. Flexible endoscope for continuous in vivo multispectral fluorescence lifetime imaging. Optics Letters, v. 38, n. 9, p. 1515-1517, 2013.

20 SHRESTHA, S. et al. High-speed multispectral fluorescence lifetime imaging implementation for in vivo applications. Optics Letters, v. 35, n. 15, p. 2558-2560, 2010.

21 PARK, J. et al. Biochemical characterization of atherosclerotic plaques by endogenous multispectral fluorescence lifetime imaging microscopy. Atherosclerosis, v. 220, n. 2, p. 394-401, 2012.

22 XIE, H. et al. Multispectral scanning time-resolved fluorescence spectroscopy (trfs) technique for intravascular diagnosis. Biomedical Optics Express, v. 3, n. 7, p. 1521-1533, 2012.

23 CHENG, S. et al. Handheld multispectral fluorescence lifetime imaging system for in vivo applications. Biomedical Optics Express, v. 5, n. 3, p. 921-931, 2014.

24 CHENG, S. Fluorescence lifetime imaging microscopy (flim) system for imaging of oral cancer and precancer. 2014. Thesis (Physics) - Texas A\&M University, College Station, 2014. 
25 CHENG, S. et al. In vivo detection of oral epithelial pre-cancer and cancer by endogenous fluorescence lifetime imaging (flim) endoscopy. Proceedings of the OSA, 2016. doi: 10.1364/CANCER.2016.CTh4A.3.

26 PARK, J. et al. A dual-modality optical coherence tomography and fluorescence lifetime imaging microscopy system for simultaneous morphological and biochemical tissue characterization. Biomedical Optics Express, v. 1, n. 1, p. 186-200, 2010.

27 JO, J. A. et al. In vivo simultaneous morphological and biochemical optical imaging of oral epithelial cancer. IEEE Transactions on Biomedical Engineering, v. 57, n. 10 p. 2596-2599, 2010.

28 JABBOUR, J. M. et al. Fluorescence lifetime imaging and reflectance confocal microscopy for multiscale imaging of oral precancer. Journal of Biomedical Optics, v. 18, n. 4, p. 046012-046012, 2013.

29 DE BEULE, P. et al. A hyperspectral fluorescence lifetime probe for skin cancer diagnosis. Review of Scientific Instruments, v. 78, n. 12, p. 123101, 2007.

30 GALLETLY, N. et al. Fluorescence lifetime imaging distinguishes basal cell carcinoma from surrounding uninvolved skin. British Journal of Dermatology, v. 159, n. 1, p. $152-161,2008$.

31 NOGUEIRA, M. S. et al. Assembly and characterization of a fluorescence lifetime spectroscopy system for skin lesions diagnostic. Proceedings of the SPIE, v. 9531, p. 95313D, 2015. doi: 10.1117/12.2180599.

32 NOGUEIRA, M. S. et al. Portable fluorescence lifetime spectroscopy system for in-situ interrogation of biological tissues. Journal of Biomedical Optics, v. 22, n. 12, p. $121608,2017$.

33 BRYDEGAARD, M. et al. Complete parameterization of temporally and spectrally resolved laser induced fluorescence data with applications in bio-photonics. Chemometrics and Intelligent Laboratory Systems, v. 142 p. 95-106, 2015.

34 BECKER, W. The bh tcspc handbook. Berlin: Becker \& Hickl, 2008.

35 BECKER, W. Advanced time-correlated single photon counting techniques. New York: Springer Science \& Business Media, 2005. (Springer series in chemical physics v. 81).

36 BECKER, W. et al. Advanced time-correlated single photon counting techniques for spectroscopy and imaging in biomedical systems. Proceedings of the SPIE, v. 5340, p. 104-112, 2004. doi: 10.1117/12.529143. 
37 ARFKEN, G. B. Mathematical methods for physicists. 7th ed. Oxford: Academic press, 2013.

38 HALL, M. Resolution and uncertainty in spectral decomposition. First Break, v. 24, n. 12 , p. $43-47,2006$.

39 GABOR, D. Theory of communication. part 1: The analysis of information. Journal of the Institution of Electrical Engineers-Part III: Radio and Communication Engineering, v. 93, n. 26, p. 429-441, 1946.

40 DIGMAN, M. A. et al. The phasor approach to fluorescence lifetime imaging analysis. Biophysical Journal, v. 94, n. 2, p. L14-L16, 2008.

41 WARE, W. R.; DOEMENY, L. J.; NEMZEK, T. L. Deconvolution of fluorescence and phosphorescence decay curves. Least-squares method. The Journal of Physical Chemistry, v. 77, n. 17, p. 2038-2048, 1973.

42 GRINVALD, A.; STEINBERG, I. Z. On the analysis of fluorescence decay kinetics by the method of least-squares. Analytical Biochemistry, v. 59, n. 2, p. 583-598, 1974.

43 GAVIN, H. The levenberg-marquardt method for nonlinear least squares curve-fitting problems. Available from: 〈http://people.duke.edu/ hpgavin/ce281/lm.pdf $\rangle$. Accessible at: 19 Nov. 2017.

44 CHAN, S. P. et al. Optimized gating scheme for rapid lifetime determinations of single-exponential luminescence lifetimes. Analytical Chemistry, v. 73, n. 18, p. 4486-4490, 2001.

45 LI, D.-U. et al. On-chip, time-correlated, fluorescence lifetime extraction algorithms and error analysis. Journal of the Optical Society of America A, v. 25, n. 5, p. 1190-1198, 2008.

46 LI, D.-U.; ARLT, J.; RICHARDSON, J.; WALKER, R.; BUTS, A.; STOPPA, D.; CHARBON, E.; HENDERSON, R. Real-time fluorescence lifetime imaging system with a $32 \times 320.13 \mu \mathrm{m}$ cmos low dark-count single-photon avalanche diode array. Optics Express, v. 18, n. 10, p. 10257-10269, 2010.

47 LERAY, A. et al. Spatio-temporal quantification of fret in living cells by fast timedomain flim: a comparative study of non-fitting methods. PloS One, v. 8, n. 7, p. e69335, 2013.

48 MOON, S.; WON, Y.; KIM, D. Y. Analog mean-delay method for high-speed fluorescence lifetime measurement. Optics Express, v. 17, n. 4, p. 2834-2849, 2009. 
49 WON, Y. J.; HAN, W.-T.; KIM, D. Y. Precision and accuracy of the analog meandelay method for high-speed fluorescence lifetime measurement. Journal of the Optical Society of America A, v. 28, n. 10, p. 2026-2032, 2011.

50 LI, D. D.-U.; ARLT, J.; TYNDALL, D.; WALKER, R.; RICHARDSON, J.; STOPPA, D.; CHARBON, E.; HENDERSON, R. K. Video-rate fluorescence lifetime imaging camera with cmos single-photon avalanche diode arrays and high-speed imaging algorithm. Journal of Biomedical Optics, v. 16, n. 9, p. 096012-096012, 2011.

51 MAAREK, J.-M. I. et al. Time-resolved fluorescence spectra of arterial fluorescent compounds: Reconstruction with the laguerre expansion technique. Photochemistry and Photobiology, v. 71, n. 2, p. 178-187, 2000.

52 JO, J. et al. Novel ultra-fast deconvolution method for fluorescence lifetime imaging microscopy based on the laguerre expansion technique. In: Engineering in Medicine and Biology Society, 2004. v. 1, p. 1271-1274, 2004. doi: 10.1109/IEMBS.2004.1403402.

53 WU, G. et al. Gpu acceleration of time-domain fluorescence lifetime imaging. Journal of Biomedical Optics, v. 21, n. 1, p. 017001-017001, 2016.

54 FIELD, R. M. et al. A 100 fps, time-correlated single-photon-counting-based fluorescence-lifetime imager in $130 \mathrm{~nm}$ cmos. IEEE Journal of Solid-State Circuits, v. 49, n. 4, p. 867-880, 2014.

55 KESSLER, D. An afocal beam relay for laser xy scanning systems. Proceedings of the SPIE, v. 8215, 2012. doi: 10.1117/12.905285. 
ANNEX A - Research Ethics Committee approval 


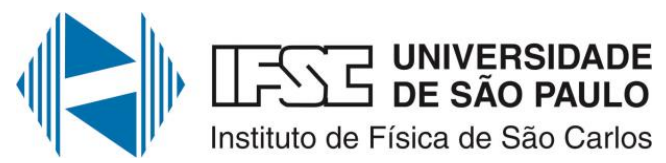

CERTIFICADO DA COMISSÃO DE ÉTICA NO USO DE ANIMAIS - CEUA/IFSC
Av. Trabalhador são-carlense, 400 / 13566-590 Caixa Postal 369 / 13560-970 São Carlos - SP, Brasil Fone: +55 16 3373-9758 www.ifsc.usp.br

Certificamos que o projeto intitulado "Otimização nos processos da formação e distribuição de PpIX no tratamento de câncer de pele por Terapia Fotodinâmica dispositivos de aplicação intradérmica", protocolo no 13/2016, sob a responsabilidade da pesquisadora Michelle Barreto Requena e sob supervisão do Prof. Dr. Vanderlei Salvador Bagnato - que envolve a produção, manutenção e/ou utilização de animais pertencentes ao filo Chordata, subfilo Vertebrata (exceto humanos), para fins de pesquisa científica - encontra-se de acordo com os preceitos da Lei ํㅜ 11.794 , de 8 de outubro de 2008, do Decreto no 6.899 , de 15 de julho de 2009, e com as normas editadas pelo Conselho Nacional de Controle da Experimentação Animal (CONCEA), e foi aprovado pela COMISSÃO DE ÉTICA NO USO DE ANIMAIS (CEUA) do Instituto de Física de São Carlos (IFSC), em reunião de 16/01/2017.

\begin{tabular}{|c|c|}
\hline Finalidade & Pesquisa Científica \\
\hline Vigência da Autorização & 16/01/2017 a 15/01/2018 (12 meses) \\
\hline Espécie/linhagem/raça & Camundongo Isogênico - Balb/c nude \\
\hline № de animais & 175 \\
\hline Peso/Idade & $15 \mathrm{~g} / 6$ semanas \\
\hline Sexo & Fêmea \\
\hline Origem & $\begin{array}{l}\text { 1. CEMIB - Centro Multidisciplinar para Investigação } \\
\text { Biológica na Área da Ciência em Animais de } \\
\text { Laboratório / UNICAMP - campus de Campinas. } \\
\text { 2. Centro de Bioterismo da Faculdade de Medicina da } \\
\text { Universidade de São Paulo / USP - campus de São } \\
\text { Paulo. }\end{array}$ \\
\hline
\end{tabular}

São Carlos, 16 de janeiro de 2017.
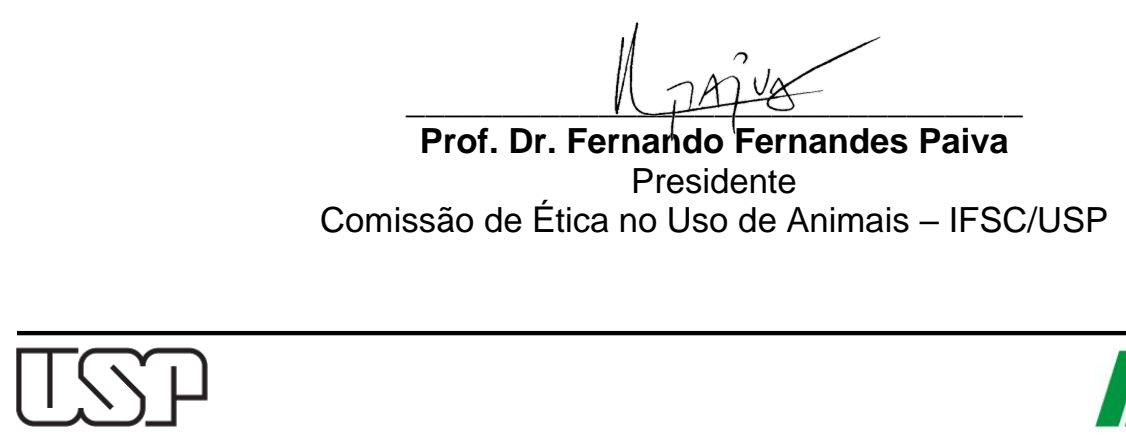
ANNEX B - Research Ethics Committee approval 


\section{PARECER DA COMISSÃO DE ÉTICA PARA O USO DE ANIMAIS - CEUA/IFSC}

Protocolo $n=03 / 2014$

A Comissão de Ética para o Uso de Animais do Instituto de Física de São Carlos - CEUA/IFSC, em reunião realizada no dia 24/06/2014, aprovou o projeto de pesquisa intitulado "Desenvolvimento de estratégias ópticas para diagnóstico e tratamento do melanoma", submetido pela pesquisadora Layla Pires. Outrossim, informamos que foi aprovada a utilizaçăo de 200 camundongos heterogênicos de linhagem $\mathrm{balb} / \mathrm{c}$ nude, com idade de 6 semanas e peso aproximado de $15 \mathrm{~g}$.

São Carlos, 30 de outubro de 2014.

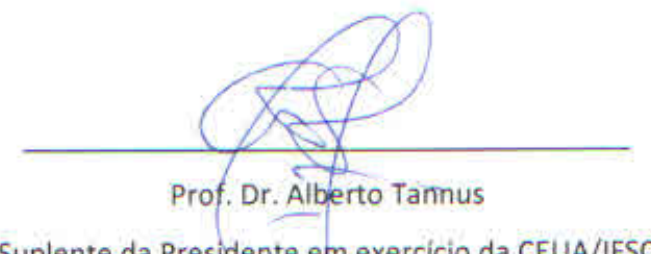

Suplente da Presidente em exercicio da CEUA/IFSC

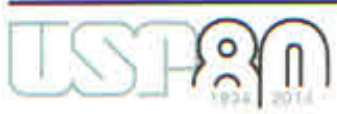

